\title{
Numerical and analytical modeling of orthogonal cutting: The link between local variables and global contact characteristics
}

\author{
A. Molinari ${ }^{\mathrm{a}, \mathrm{b}, *}$, R. Cheriguene ${ }^{\mathrm{a}, \mathrm{b}}, \mathrm{H}_{\text {. Miguelez }}^{\mathrm{a}}$ \\ a Department of Mechanical Engineering, Carlos III University- Madrid, Calle Butarque 15, 28911 Leganes, Madrid, Spain \\ ${ }^{\mathrm{b}}$ Laboratory of Physics and Mechanics of Materials, LEM3, CNRS, Paul Verlaine University-Metz, Ile du Saulcy, 57045 Metz, France
}

Keywords:

Orthogonal cutting

Friction

Sticking and sliding contact

Contact length

Thermal effects

\begin{abstract}
The response of the tool-chip interface is characterized in the orthogonal cutting process by numerical and analytical means and compared to experimental results. We study the link between local parameters (chip temperature, sliding friction coefficient, tool geometry) and overall friction characteristics depicting the global response of the tool-chip interface. Sticking and sliding contact regimes are described.

The overall friction characteristics of the tool are represented by two quantities: (i) the mean friction coefficient qualifies the global response of the tool rake face (tool edge excluded) and (ii) the apparent friction coefficient reflects the overall response of the entire tool face, the effect of the edge radius being included. When sticking contact is dominant the mean friction coefficient is shown to be essentially the ratio of the average shear flow stress along the sticking zone by the average normal stress along the contact zone. The dependence of overall friction characteristics is analyzed with respect to tool geometry and cutting conditions. The differences between mean friction and apparent friction are quantified. It is demonstrated that the evolutions of the apparent and of the mean friction coefficients are essentially controlled by thermal effects. Constitutive relationships are proposed which depict the overall friction characteristics as functions of the maximum chip temperature along the rake face. This approach offers a simple way for describing the effect of cutting conditions on the tool-chip interface response. Finally, the contact length and contact forces are analyzed. Throughout the paper, the consistency between numerical, analytical and experimental results is systematically checked.
\end{abstract}

\section{Introduction}

A salient issue in the modeling of machining is to reach a better perception of the relationship between local parameters (stress, strains, temperature, velocity fields, local friction characteristics) and global variables (overall friction characteristics, shear angle, chip morphology, forces, and contact length). Clarifying this connection is especially important for the validation of the physical concepts introduced into the modeling and for improving analytical models of machining.

The aim of this work is to investigate the relationship between local and global cutting parameters by developing a fruitful interplay between theoretical tools offered by numerical methods on the one hand and analytical formulations on the other hand. The relationship between local variables and global friction characteristics is analyzed in a first step. Then, the tool-chip contact length and the contact forces exerted on the tool are characterized.

\footnotetext{
* Corresponding author at: Laboratory of Physics and Mechanics of Materials, LEM3, CNRS, Paul Verlaine University-Metz, Ile du Saulcy, 57045 Metz, France. Tel.: + 33 387315369; fax: + 33387315366

E-mail address: molinari@lpmm.univ-metz.fr (A. Molinari).
}

The use of Finite Element methods for the analysis of orthogonal cutting has been initiated three decades ago [1,2]. Presently, most of simulations are relying either on Lagrangian formulations with automatic remeshing to avoid using a separation criterion [3,4], or on the Arbitrary Lagrangian Eulerian (ALE) technique [5,6]. Conversely, since the pioneered work of Merchant [7] dealing with perfectly plastic materials, a thermo-mechanical framework has been developed for the analytical modeling of cutting [8-11]. A foremost aspect of the present work is to establish a dialog between numerical modeling of machining that provides an efficient tool for analyzing local and global variables and analytical formulation that offers a clear synthetic perception of the phenomena studied.

The work material is taken to be a medium carbon steel 42CrMo4. The thermo-viscoplastic response of the material is modeled with a Johnson-Cook law. Material parameters are fixed, but interface properties are varied since the analysis of frictional effects is of particular concern.

In machining, the contact problem is generally modeled with phenomenological laws that include sticking and sliding contact regimes [12-18]. Important features of dry contact can be captured by using the Coulomb friction law. Sometimes, a modified Coulomb friction law has been adopted, which accounts for the saturation at a given value of the shear stress on the tool face. In the present 


\begin{tabular}{|c|c|c|c|}
\hline \multicolumn{2}{|c|}{ Nomenclature } & $l_{p}$ & sticking length \\
\hline \multirow{2}{*}{\multicolumn{2}{|c|}{ Cutting conditions }} & $\eta$ & part of frictional heat transferred to the chip \\
\hline & & $\kappa$ & thermal conductance of the tool-chip interface \\
\hline$V$ & cutting speed & $T_{1}$ & $\begin{array}{l}\text { temperature of the work-material at the entry of the } \\
\text { flat part of the rake face }\end{array}$ \\
\hline$t_{1}$ & uncut chip thickness & $\bar{T}_{i n t}$ & mean temperature along the tool rake face \\
\hline$\alpha$ & rake angle & $T_{\max }$ & maximum temperature of the chip along the rake face \\
\hline$\gamma$ & clearance angle & \multirow{2}{*}{\multicolumn{2}{|c|}{ Material characteristics of the work material }} \\
\hline$R$ & cutting edge radius & & \\
\hline \multirow{2}{*}{\multicolumn{2}{|c|}{ Chip characteristics }} & $T_{r}$ & reference temperature in the Johnson-Cook law \\
\hline & & $T_{m}$ & melting temperature \\
\hline$t_{2}$ & chip thickness & $\dot{\varepsilon}_{0}$ & reference strain rate \\
\hline & shear angle & $\rho$ & mass density \\
\hline \multirow{2}{*}{\multicolumn{2}{|c|}{$\begin{array}{l}\phi_{A} \text { and } \phi^{*} \text { alternative representations of the shear angle (see } \\
\text { Fig. } 1 \mathrm{~b} \text { ) } \\
\bar{\tau}_{P Z} \quad \begin{array}{l}\text { mean shear flow stress along the central line of the } \\
\text { primary shear zone }\end{array}\end{array}$}} & $\begin{array}{l}C_{p} \\
k\end{array}$ & $\begin{array}{l}\text { heat capacity per unit mass } \\
\text { heat conductivity }\end{array}$ \\
\hline & & $\beta$ & Taylor-Quinney coefficient \\
\hline \multicolumn{2}{|c|}{ Tool-chip interface } & \multicolumn{2}{|c|}{ Forces } \\
\hline & & & cutting force \\
\hline & sliding friction coefficient & & thrust force \\
\hline \multirow{2}{*}{\multicolumn{2}{|c|}{$\begin{array}{l}\bar{\lambda}=\arctan (\bar{\mu}) \text { mean friction angle } \\
\mu_{a p} \quad \text { apparent friction coefficient }\left(=\left(F_{T} / F_{C}\right) \text { when } \alpha=0\right)\end{array}$}} & \multirow{2}{*}{\multicolumn{2}{|c|}{$\begin{array}{l}F_{n}(\text { rake }) \text { and } F_{t}(\text { rake }) \text { normal and tangential forces exerted by } \\
\text { the chip on the flat part of the rake face }\end{array}$}} \\
\hline & & & \\
\hline \multicolumn{2}{|c|}{$\begin{array}{l}\lambda_{a p}=\arctan \left(\mu_{a p}\right) \text { apparent friction angle } \\
V_{\varsigma} \quad \text { sliding velocity }\end{array}$} & \multirow{2}{*}{\multicolumn{2}{|c|}{ Field variables }} \\
\hline & & & \\
\hline$\tau$ & $\begin{array}{l}\text { snear stress } \\
\text { normal stress }\end{array}$ & $d_{i j}^{p}$ & plastic strain rate tensor \\
\hline & exponent controlling the decay of the normal stress & $\sigma_{i j}$ & Cauchy stress tensor \\
\hline$\tau_{Y}$ & local value of the shear flow stress & & absolute temperature \\
\hline & average shear flow stress along the sticking zone & $\sigma_{e q}$ & Mises equivalent stress \\
\hline & contact length on the flat part of the rake face & $\dot{\varepsilon}_{e q}$ & equivalent strain rate \\
\hline
\end{tabular}

paper, the original formulation of the Coulomb law is used. The shear stress at the rake face is naturally limited by the shear flow stress of the work material when sticking contact occurs. The effects of the sliding friction coefficient and of cutting conditions (cutting speed, uncut chip thickness, tool edge radius) on local and global variables are analyzed and we examine how these variables are related together. Of particular interest are the analytical relationships derived for global friction characteristics and for the tool-chip contact length in terms of cutting conditions and local field variables. These relationships provide conceptual models that allow us to clarify the physical mechanisms governing the cutting problem. They also offer a way to extract useful information from numerical experiments for the purpose of improving analytical models of machining.

The paper is organized as follows. The theoretical framework of the cutting model is presented in Section 2. In Section 3 numerical results obtained by the ALE Finite Element method are correlated to experimental data. A parametric analysis is also performed by varying the values of the sliding friction coefficient, the cutting speed, the uncut chip thickness, the tool edge radius, the coefficient of conversion of the plastic work into heat and the thermal resistance of the tool-chip interface. Two global characteristics of the tool-chip friction are introduced in Section 4. A mean friction coefficient $\bar{\mu}$ is defined which characterizes the overall frictional response of the tool rake face (tool edge and clearance contact excluded). Conversely, the apparent friction coefficient $\mu_{a p}$ accounts for the totality of the tool-chip contact and is obtained from the cutting and thrust forces. The dependence of the mean friction coefficient $\bar{\mu}$ with respect to the sliding friction coefficient $\mu$ and cutting conditions is analyzed in Section 5. Effects of thermo- mechanical coupling and of thermal softening are quantified. The transition towards a contact regime dominated by sticking is examined and the evolution of $\bar{\mu}$ is described by analytical means and correlated to numerical results. The dependence of the apparent friction coefficient $\mu_{a p}$ with respect to $\mu$ and cutting conditions is investigated in Section 6. In particular, the role of the tool edge radius is analyzed and the evolutions of $\mu_{a p}$ and $\bar{\mu}$ are compared when cutting conditions are varied. Effects of the cutting speed and of the uncut chip thickness are correlated to experimental data. In Section 7 it is shown that the dependence of $\bar{\mu}$ with respect to the cutting speed and the uncut chip thickness can be essentially expressed in terms of a temperature characterizing the heating of the chip along the tool rake face. A similar result is obtained for the apparent friction coefficient $\mu_{a p}$. Analytical forms are proposed for the evolution of $\bar{\mu}$ and $\mu_{a p}$. The contact length and contact forces are investigated, respectively, in Sections 8 and 9. Conclusions are drawn in Section 10.

\section{Physical assumption and numerical framework}

The physical assumptions and the numerical framework adopted for the modeling of orthogonal cutting are presented in this section.

\subsection{Thermo-mechanical response of the work material}

The work material is a medium carbon steel 42CrMo4 (AFNOR: 42CD4) with chemical composition given in Sutter and Molinari [19]. The thermo-mechanical response is represented by the 
Johnson-Cook law:

$\sigma_{e q}=\left[A+B \varepsilon_{e q}^{n}\right]\left[1+C \ln \left(\frac{\dot{\varepsilon}_{e q}}{\dot{\varepsilon}_{0}}\right)\right]\left[1-\left(\frac{T-T_{r}}{T_{m}-T_{r}}\right)^{m}\right]$

Here $\sigma_{e q}$ and $\dot{\varepsilon}_{e q}$ are, respectively, the Mises equivalent stress and strain rate, $T$ is the absolute temperature, $T_{r}$ is a reference temperature (room temperature), $T_{m}$ is the melting temperature, $\dot{\varepsilon}_{0}$ is a reference strain rate. The plastic flow is assumed to be governed by the $J_{2}$-flow theory.

The parameters associated to the $42 \mathrm{CrMo} 4$ steel were identified experimentally (quasi-static tests were conducted on a universal hydraulic machine and dynamic tests on split Hopkinson bars) by Molinari et al. [20] and are reported in Table 1. Other mechanical and thermal properties of the work material and of the tool are given in Table 2 . The tool is supposed to behave elastically.

The evolution of the temperature in the work material is governed by the energy equation:

$\rho C_{p} \dot{T}-k \Delta T=\beta d_{i j}^{p} \sigma_{i j}$

$\dot{T}$ is the material derivative of the temperature, $\Delta T$ is the Laplacian of $T . \rho, C_{p}$ and $k$ are, respectively, the mass density, the heat capacity per unit mass (at constant pressure) and the heat conductivity of the work material. The right hand side of Eq. (2) represents the proportion $\beta$ of the plastic work converted into heat. $d_{i j}^{p}$ and $\sigma_{i j}$ are, respectively, the components of the plastic strain rate tensor and of the Cauchy stress tensor. The Taylor-Quinney coefficient $\beta$ is taken as constant. The value $\beta=0.9$ will be adopted as reference value but variations of $\beta$ will be also considered. The thermal properties $C_{p}$ and $k$ are considered to be independent of the temperature.

Frictional heating occurs at the tool-chip interface along the sliding zone. The rate of frictional energy per unit surface is equal to $\tau V_{S}$, where $\tau$ is the shear stress and $V_{S}$ is the sliding velocity. The frictional energy is assumed to be totally converted into heat. The part of frictional heat transferred to the chip is denoted by $\eta$ $(0 \leq \eta \leq 1)$. In the present paper, it is assumed that frictional heat is shared equally between the two bodies in contact $(\eta=0.5)$. The thermal conductance of the tool-chip interface has the value $\kappa=2000 \mathrm{Wm}^{-2} \mathrm{~K}^{-1}$. The rate of heat energy transferred to the chip is governed by the values of $\kappa$ and $\eta$ and by the thermal properties of the two bodies in contact.

The free surface of the workpiece and of the chip and the external boundary of the tool are assumed to be adiabatically insulated. The dimension of the tool is taken large enough, so that the heating at the tool-chip interface is not affected by the thermal conditions at the external boundary of the tool.

Table 1

Material parameters of the Johnson-Cook model for the $42 \mathrm{CrMo} 4$ steel, according to [20].

\begin{tabular}{llllllll}
\hline$A(\mathrm{MPa})$ & $B(\mathrm{MPa})$ & $C$ & $n$ & $\dot{\varepsilon}_{0}\left(\mathrm{~S}^{-1}\right)$ & $m$ & $T_{r}(\mathrm{~K})$ & $T_{m}(\mathrm{~K})$ \\
\hline 612 & 436 & 0.008 & 0.15 & $5.77 \mathrm{E}-4$ & 1.46 & 293 & 1793 \\
\hline
\end{tabular}

Table 2

Mechanical and thermal parameters of the work-material (42CrMo4 steel) and of the carbide tool.

\begin{tabular}{lrrrrrc}
\hline & $E(\mathrm{GPa})$ & $v$ & $\rho\left(\mathrm{kg} \mathrm{m}^{-3}\right)$ & $C_{p}\left(\mathrm{~m}^{2} \mathrm{~s}^{-2} \mathrm{~K}^{-1}\right)$ & $k\left(\mathrm{NK}^{-1} \mathrm{~s}^{-1}\right)$ & $\beta$ \\
\hline Work material & 202 & 0.3 & 7800 & 500 & 54 & 0.9 \\
Tool & 1000 & 0.3 & 12700 & 234 & 33.5 &. \\
\hline
\end{tabular}

\subsection{Friction law}

The Coulomb friction law is used. Under sliding conditions the shear stress $\tau$ is related to the normal stress $\sigma$ by

$\tau=\mu \sigma$

where $\mu$ is the sliding friction coefficient. $\mu$ is considered to be constant along the interface and to be independent from cutting conditions. Sticking contact is activated when $\tau<\mu \sigma$. In machining, this situation occurs at the vicinity of the tool tip where high values of the normal stress $\sigma$ are reached. In all simulations, it has been verified that under sticking condition the shear stress on the toolchip interface is identical, as a consequence of the $J_{2}$-flow theory, to the local value of the shear flow stress $\tau_{Y}$ of the work material. Thus, the contact law at the tool-chip interface can be summarized as

$\tau=\inf \left(\tau_{Y}, \mu \sigma\right)$

where $\tau_{Y}$ is function of temperature, strain and strain rate.

The contact model (4) was introduced by Zorev [15] and was implemented in Finite Element simulations of orthogonal cutting, see for example Masurich and Ortiz [4]. Arrazola and Özel [18] have recently explored the effect of a limit shear stress $\tau_{\text {limit }}$ different from $\tau_{Y}$ in the contact law. It is worth emphasizing that in the present modeling there was no necessity to introduce any limiting stress in the contact law. The limitation by the flow stress $\tau_{Y}$ along the sticking zone appears just as an outcome of the calculations.

\subsection{Numerical model}

Finite Element simulations are performed by using the code ABAQUS/Explicit [21] and the ALE formulation. Specifications related to the ALE formulation at the boundaries of the workpiece and of the chip are shown in Fig. 1a. Geometrical parameters are defined in Fig. 1b. Cutting conditions are reported in Table 3.

Only stationary flow and continuous chip formation are simulated in the present framework. Plane-strain deformations are assumed. An example of mesh configuration is displayed in Fig. 1a. CPE4RT quadrilateral elements with reduced integration were used. The contact problem was simulated by using the penalty (and surface/surface) algorithm provided by the FE code. It was checked that consistent results were obtained with this approach (i.e. the contact law (4) was never violated). The mesh size was about $4 \mu \mathrm{m}$.

\section{Numerical results with constant values of the sliding friction coefficient}

All results presented in this paper are related to quasi-steadystate regimes obtained when the interface temperature and the values of cutting forces are nearly time independent.

\subsection{Cutting and thrust forces: correlation with experimental data}

Before commencing a detailed analysis of the orthogonal cutting process, it is worth evaluating the model's capabilities by comparing numerical simulations of the cutting and thrust forces (per unit cutting width), respectively, $F_{C}$ and $F_{T}$, with experimental measurements, see Fig. 2a and $b$. The rake angle is $\alpha=0$. Orthogonal cutting tests were performed under dry conditions on a CNC lathe by Devillez et al. [22] for the $42 \mathrm{CrMo} 4$ steel whose mechanical characteristics are given in Section 2.1. An uncoated carbide insert without chip-breaker groove was used. It must be noted that the identical material (same delivery) was used on the one hand for machining experiments [22] and on the other hand for characterizing the constitutive response by mechanical testing [20]. This 


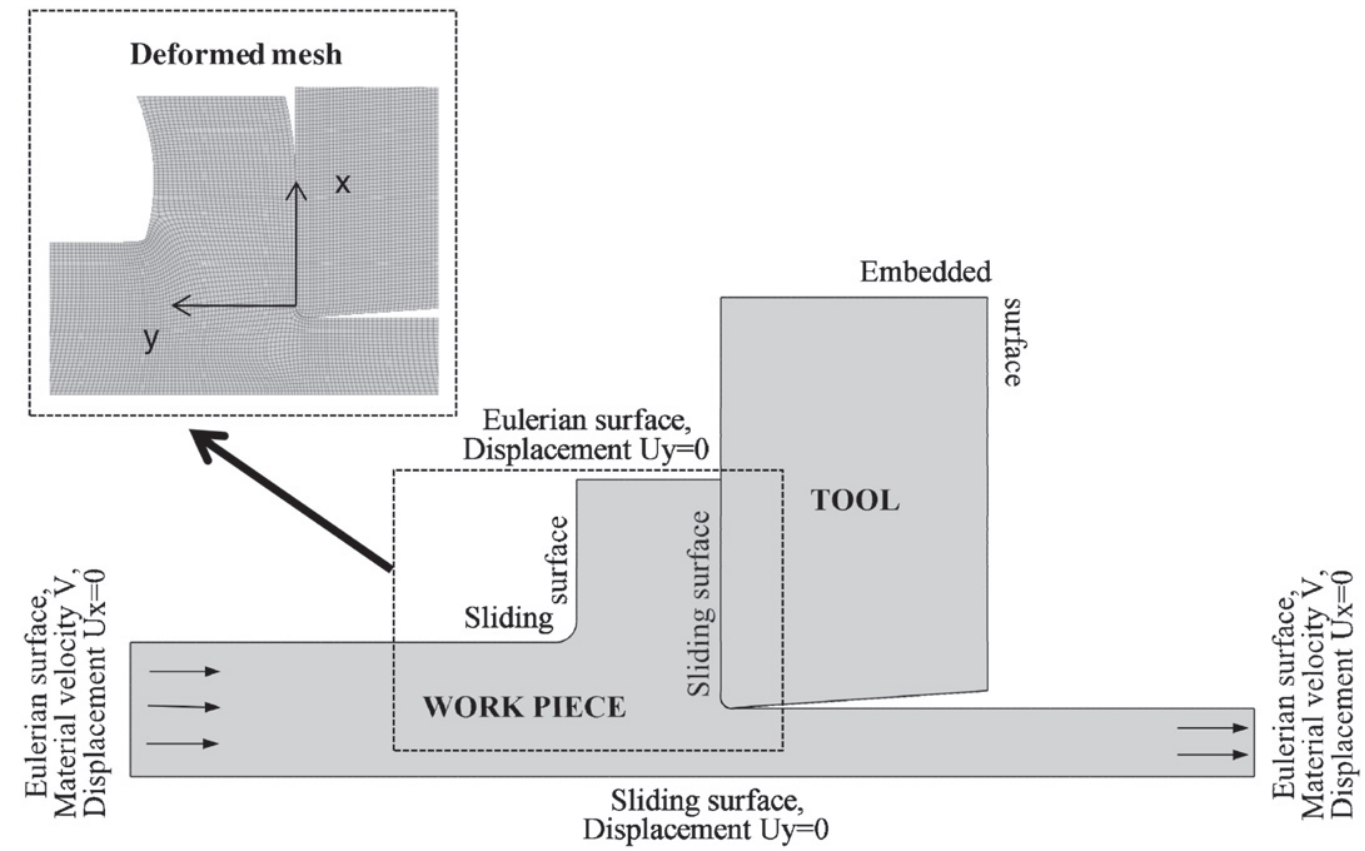

b

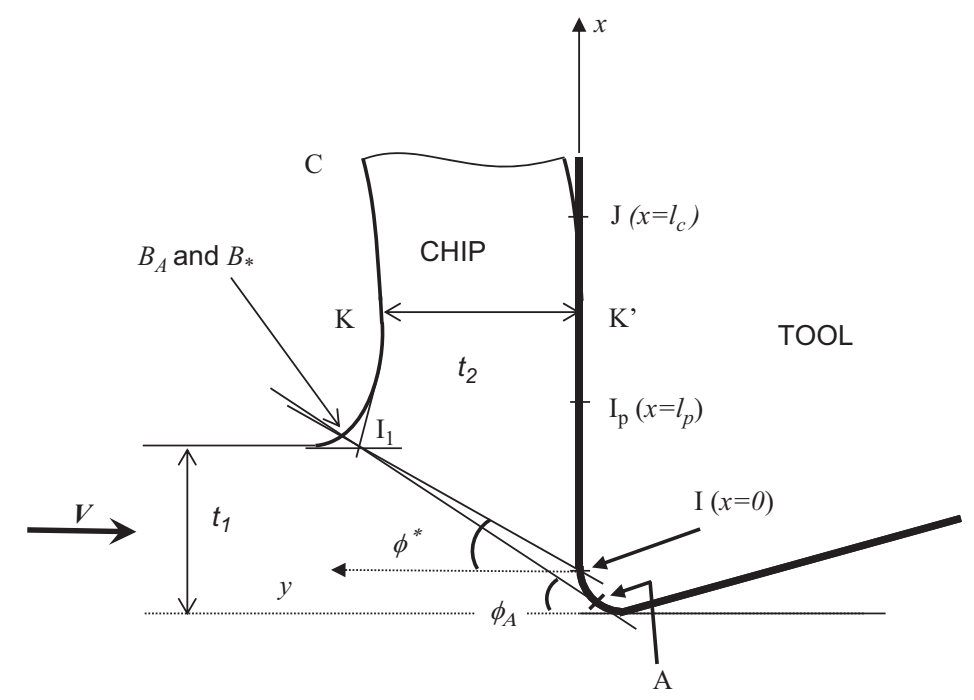

Fig. 1. (a) Boundary conditions of the ALE orthogonal cutting model. An example of mesh configuration is shown in the upper left part. (b) Geometrical characteristics of the problem for the rake angle $\alpha=0$.

\section{Table 3}

Values of the cutting parameters used in simulations; $\alpha$ rake angle, $\gamma$ clearance angle, $t_{1}$ uncut chip thickness, $R$ cutting edge radius, $V$ cutting velocity, $\kappa$ thermal conductance of the tool-chip interface, $\eta$ heat partition coefficient.

\begin{tabular}{lllllll}
\hline$\alpha$ & $\gamma$ & $t_{1}$ & $R$ & $V$ & $\kappa$ & $\eta$ \\
\hline 0 & $7^{\circ}$ & $\begin{array}{l}0.05 \mathrm{~mm} \\
\leq t_{1} \leq 0.5 \mathrm{~mm}\end{array}$ & 0.015 and $0.030 \mathrm{~mm}^{1}$ & $\begin{array}{l}1 \mathrm{~ms}^{-1} \leq V \\
\leq 50 \mathrm{~ms}^{-1}\end{array}$ & $2000 \mathrm{Wm}^{-2} \mathrm{~K}^{-1}$ & 0.5 \\
& & & &
\end{tabular}

steel is taken as the reference work material in all simulations of the present paper.

The apparent friction coefficient $\mu_{a p}=F_{T} / F_{C}$ (general definition given by Eq. (5)) is shown in terms of the cutting speed in Fig. 2c. The results shown in Fig. 2 indicate a good correlation between modeling and experimental data for the value $\mu=0.8$ of the sliding friction coefficient. In the simulations, two values of the cutting edge radius were used, $R=15$ and $30 \mu \mathrm{m}$. The results for the cutting force are weakly affected by $R$. The effect of $R$ on the thrust force is larger.

\subsection{Parametric analysis}

A parametric analysis is conducted by considering various values of $\mu$ from 0 to 1 and cutting speeds in the range $1 \mathrm{~ms}^{-1} \leq V \leq 50 \mathrm{~ms}^{-1}$. Other parameters are varied as indicated in Table 3. The values of the rake and clearance angles are fixed $\left(\alpha=0, \gamma=7^{\circ}\right)$.

Results of numerical simulations are reported in Table 4 for the uncut chip thickness $t_{1}=0.1 \mathrm{~mm}$, the cutting edge radius $R=0.015$ $\mathrm{mm}$ and the thermal conductance of the tool-chip interface $\kappa=2000 \mathrm{Wm}^{-2} \mathrm{~K}^{-1}$. Results include the chip thickness $t_{2}$, the contact length $l_{c}$ (see Fig. $1 \mathrm{~b}$ ), the sticking ratio $l_{p} / l_{c}\left(l_{p}\right.$ is the 

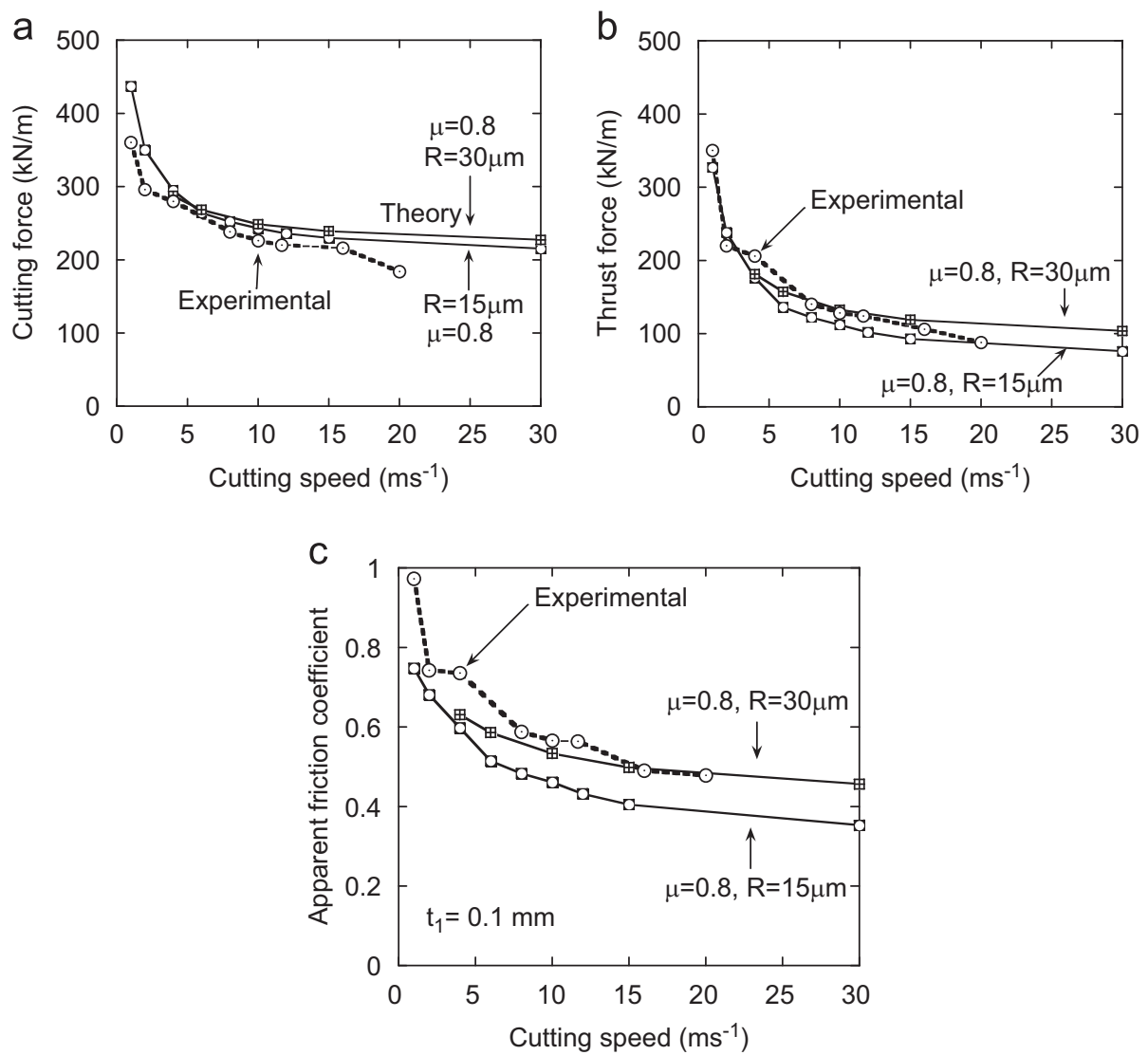

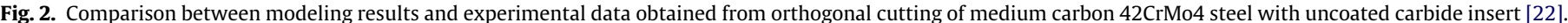

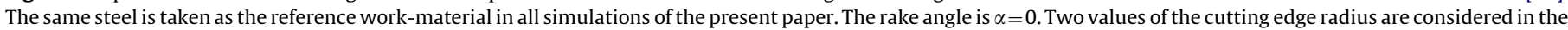

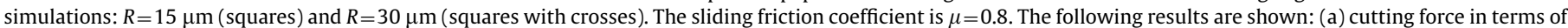
cutting speed, (b) thrust force in terms of cutting speed, and (c) apparent friction coefficient $\mu_{a p}$ (thrust force divided by the cutting force).

sticking length on the rake face, see Fig. $1 \mathrm{~b})$, the average value $\bar{\tau}_{P Z}$ of the shear flow stress along the central line of the primary shear zone (see Appendix A), the average shear stress $\bar{\tau}_{S Z}$ along the rakeface sticking zone $0 \leq x \leq l_{p}$, the cutting and thrust forces per unit cutting width $F_{C}$ and $F_{T}$ and the apparent friction coefficient $\mu_{a p}$. In Table 4 are also reported the components $F_{n}\left(\right.$ rake) and $F_{t}$ (rake) of the force exerted by the chip on the part $I J\left(0 \leq x \leq l_{c}\right)$ of the tool, see Fig. 1b. The lower indices $(n)$ and $(t)$ refer, respectively, to the normal and tangential components to the tool. The mean friction coefficient associated to the contact zone $I J$ is defined by Eq. (7). The mean shear flow stress along the sticking zone, $\bar{\tau}_{S Z}=\left(1 / l_{p}\right)$ $\int_{0}^{l_{p}} \tau(x) d x$, is calculated by taking the nodal values of $\tau$ on a path following the tool-chip interface.

The temperature $T_{1}$ is the nodal temperature of the workmaterial at $I(x=0) . T_{\max }$ is the maximum nodal temperature of the work-material along the tool-chip interface.

The shear angle $\phi=\arctan \left(t_{1} / t_{2}\right)$ and other angles $\phi_{A}$ and $\phi^{*}$ (defined in Fig. 1b) are also reported. The point $I_{1}$ in Fig. $1 \mathrm{~b}$ used to characterize $\phi_{A}$ and $\phi^{*}$ is defined in Appendix C. The chip thickness $t_{2}$ is evaluated at the point $K$ taken on the chip free surface where the tangent is parallel to the tool rake-face, see Fig. $1 \mathrm{~b} ; t_{2}$ is the distance $K K^{\prime}$ with the tool rake face, where $K^{\prime}$ is the orthogonal projection of $K$ on the rake face.

The effect of the tool edge radius is evaluated in Table 5 for $R=0.03 \mathrm{~mm}$ while the value $R=0.015 \mathrm{~mm}$ was considered in Table 4 . Other parameters are: $t_{1}=0.1 \mathrm{~mm}, \kappa=2000 \mathrm{Wm}^{-2} \mathrm{~K}^{-1}$ and $\mu=0.8$. The effect of the uncut chip thickness $t_{1}$ is analyzed in Table 6 for $R=0.015 \mathrm{~mm}, \kappa=2000 \mathrm{Wm}^{-2} \mathrm{~K}^{-1}$ and $\mu=0.6$. Finally, the effect of the Taylor-Quinney coefficient $\beta$ has been quantified for $t_{1}=0.1 \mathrm{~mm}, R=0.015 \mathrm{~mm}, \mu=0.6$ and $\kappa=2000 \mathrm{Wm}^{-2} \mathrm{~K}^{-1}$. The corresponding results are reported later.

\section{Global friction characteristics}

The contact between tool and chip can be globally characterized in terms of an apparent friction coefficient $\mu_{a p}$. Considering the total force $R$ exerted by the workpiece onto the tool, $\mu_{a p}$ is defined as the ratio of the components of $R$ tangential and normal to the tool rake face. With respect to the cutting and thrust forces, respectively, $F_{C}$ and $F_{T}, \mu_{a p}$ can be expressed as

$\mu_{a p}=\frac{F_{C} \tan \alpha+F_{T}}{F_{C}-F_{T} \tan \alpha}$

In this paper $\alpha=0$, therefore $\mu_{a p}$ is simply the ratio of the thrust and cutting forces.

The apparent friction coefficient is an important aspect in the analytical modeling of cutting. Together with the shear flow stress along the primary shear zone (PSZ) and the shear angle (inclination of the PSZ) it allows one to determine the cutting and thrust forces by analytical means. Therefore it is worth exploring the effects of material properties, of interface characteristic and of cutting conditions on $\mu_{a p}$ and to seek whether this coefficient could be characterized in terms of a constitutive law. In general $\mu_{a p}$ is different from $\mu$ as soon as sticking between chip and tool is activated. Indeed, effects of sliding friction and sticking contact are merged into $\mu_{a p}$. The apparent friction coefficient is also influenced by the contact forces exerted on the rounded part of the tool edge. 
Table 4

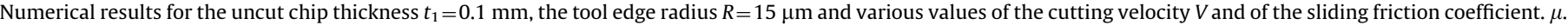

$t_{1}=0.1 \mathrm{~mm}, \kappa=2000 \mathrm{Wm}^{-2} \mathrm{~K}^{-1}, R=0.015 \mathrm{~mm}, \alpha=0^{\circ}, \gamma=7^{\circ}, \beta=0.9$

\begin{tabular}{lllllllllllllllllllllll}
\hline$\#$ & $\mu$ & $V$ & $F_{C}$ & $F_{T}$ & $\mu_{a p}$ & $F_{n}$ (rake) & $F_{t}($ rake) & $\bar{\mu}$ & $\bar{\tau}_{P Z}$ & $\bar{\tau}_{S Z}$ & $t_{2}$ & $l_{c}$ & $l_{p} / l_{c}$ & $\phi$ & $\phi_{A}$ & $\phi^{*}$ & $T_{\max }$ & $T_{1}$ & $X_{\max } / l_{c}$
\end{tabular}

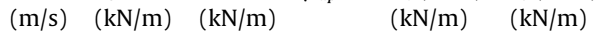

\begin{tabular}{|c|c|c|c|c|c|c|c|c|c|c|c|c|c|c|c|c|c|c|c|}
\hline 01 & 0.0 & 1 & 186 & 27.0 & 0.145 & 166 & 0 & 0 & 647 & $X$ & 0.231 & 0.163 & 0 & 23.4 & 23.4 & 21.0 & 656 & 656 & 0 \\
\hline 02 & 0.0 & 2 & 179 & 29.0 & 0.162 & 157 & 0 & 0 & 647 & $\mathrm{X}$ & 0.217 & 0.155 & 0 & 24.7 & 24.5 & 22.0 & 692 & 692 & 0 \\
\hline 03 & 0.0 & 4 & 175 & 28.0 & 0.160 & 148 & 0 & 0 & 647 & $X$ & 0.205 & 0.147 & 0 & 26.0 & 25.3 & 23.4 & 748 & 748 & 0 \\
\hline 04 & 0.0 & 6 & 171 & 29.0 & 0.170 & 145 & 0 & 0 & 652 & $X$ & 0.199 & 0.142 & 0 & 26.7 & 26.1 & 24.2 & 787 & 787 & 0 \\
\hline 05 & 0.0 & 8 & 172 & 28.0 & 0.163 & 144 & 0 & 0 & 652 & $X$ & 0.195 & 0.141 & 0 & 27.1 & 26.2 & 24.5 & 789 & 789 & 0 \\
\hline 06 & 0.0 & 10 & 172 & 28.0 & 0.163 & 143 & 0 & 0 & 652 & $X$ & 0.191 & 0.142 & 0 & 27.6 & 26.9 & 24.2 & 796 & 796 & 0 \\
\hline 07 & 0.0 & 12 & 172 & 28.5 & 0.166 & 143 & 0 & 0 & 654 & $X$ & 0.189 & 0.137 & 0 & 27.9 & 27.0 & 24.4 & 808 & 808 & 0 \\
\hline 08 & 0.0 & 15 & 171 & 30.5 & 0.178 & 142 & 0 & 0 & 655 & $X$ & 0.183 & 0.136 & 0 & 28.7 & 27.9 & 25.3 & 820 & 820 & 0 \\
\hline 09 & 0.0 & 30 & 170 & 38.0 & 0.224 & 141 & 0 & 0 & 661 & $X$ & 0.162 & 0.128 & 0 & 31.7 & 30.5 & 28.5 & 850 & 850 & 0 \\
\hline 10 & 0.0 & 50 & 169 & 39.0 & 0.231 & 142 & 0 & 0 & 664 & $X$ & 0.170 & 0.129 & 0 & 30.5 & 29.8 & 27.2 & 899 & 899 & 0 \\
\hline 11 & 0.1 & 1 & 202 & 44.0 & 0.218 & 179 & 17.9 & 0.100 & 642 & $X$ & 0.238 & 0.194 & 0 & 22.8 & 22.4 & 20.3 & 690 & 673 & 0.538 \\
\hline 12 & 0.1 & 2 & 192 & 45.8 & 0.238 & 169 & 16.9 & 0.100 & 641 & $X$ & 0.220 & 0.180 & 0 & 24.5 & 23.9 & 21.6 & 740 & 713 & 0.648 \\
\hline 13 & 0.1 & 4 & 188 & 43.8 & 0.233 & 158 & 15.8 & 0.100 & 641 & $\mathrm{X}$ & 0.206 & 0.173 & 0 & 25.9 & 25.3 & 22.4 & 793 & 769 & 0.673 \\
\hline 14 & 0.1 & 6 & 186 & 45.7 & 0.245 & 157 & 15.7 & 0.100 & 644 & $X$ & 0.204 & 0.169 & 0 & 26.2 & 25.4 & 23.6 & 832 & 809 & 0.726 \\
\hline 15 & 0.1 & 8 & 187 & 45.3 & 0.242 & 156 & 15.7 & 0.100 & 647 & $X$ & 0.200 & 0.168 & 0 & 26.6 & 25.5 & 22.9 & 856 & 813 & 0.723 \\
\hline 16 & 0.1 & 10 & 188 & 47.6 & 0.254 & 158 & 15.8 & 0.100 & 647 & $\mathrm{X}$ & 0.205 & 0.168 & 0 & 26.0 & 25.2 & 22.5 & 887 & 839 & 0.724 \\
\hline 17 & 0.1 & 12 & 188 & 47.8 & 0.255 & 157 & 15.7 & 0.100 & 648 & $X$ & 0.203 & 0.168 & 0 & 26.2 & 25.4 & 22.4 & 905 & 854 & 0.724 \\
\hline 18 & 0.1 & 15 & 188 & 47.7 & 0.254 & 157 & 15.7 & 0.100 & 650 & $X$ & 0.201 & 0.168 & 0 & 26.4 & 25.5 & 22.9 & 933 & 874 & 0.724 \\
\hline 19 & 0.1 & 30 & 191 & 47.9 & 0.251 & 157 & 15.7 & 0.100 & 655 & $X$ & 0.194 & 0.168 & 0 & 27.2 & 26.4 & 23.8 & 1025 & 936 & 0.755 \\
\hline 20 & 0.1 & 50 & 191 & 47.8 & 0.251 & 157 & 15.7 & 0.100 & 658 & $X$ & 0.190 & 0.163 & 0 & 27.7 & 28.2 & 25.8 & 1094 & 980 & 0.744 \\
\hline 21 & 0.2 & 1 & 227 & 64.0 & 0.282 & 199 & 39.9 & 0.200 & 615 & $\mathrm{X}$ & 0.265 & 0.200 & 0 & 20.7 & 19.9 & 18.1 & 738 & 707 & 0.659 \\
\hline 22 & 0.2 & 2 & 212 & 64.0 & 0.303 & 186 & 37.1 & 0.200 & 624 & $X$ & 0.234 & 0.188 & 0 & 23.1 & 22.0 & 20.0 & 802 & 743 & 0.730 \\
\hline 23 & 0.2 & 4 & 204 & 65.5 & 0.322 & 177 & 35.3 & 0.200 & 632 & $X$ & 0.217 & 0.174 & 0 & 24.7 & 24.0 & 22.1 & 874 & 790 & 0.731 \\
\hline 24 & 0.2 & 6 & 203 & 66.0 & 0.326 & 167 & 33.3 & 0.200 & 638 & $\mathrm{X}$ & 0.201 & 0.163 & 0 & 26.5 & 25.5 & 23.4 & 928 & 819 & 0.777 \\
\hline 25 & 0.2 & 8 & 202 & 65.5 & 0.324 & 173 & 34.7 & 0.200 & 647 & $X$ & 0.204 & 0.164 & 0 & 26.1 & 25.4 & 23.2 & 980 & 854 & 0.775 \\
\hline 26 & 0.2 & 10 & 203 & 65.5 & 0.323 & 176 & 35.2 & 0.200 & 647 & $X$ & 0.216 & 0.175 & 0 & 24.8 & 23.9 & 21.9 & 1027 & 893 & 0.821 \\
\hline 27 & 0.2 & 12 & 203 & 66.0 & 0.325 & 175 & 35.1 & 0.200 & 647 & $\mathrm{X}$ & 0.212 & 0.170 & 0 & 25.3 & 24.2 & 22.1 & 1061 & 912 & 0.753 \\
\hline 28 & 0.2 & 15 & 203 & 66.4 & 0.328 & 175 & 34.9 & 0.200 & 647 & $X$ & 0.208 & 0.170 & 0 & 25.7 & 24.7 & 22.7 & 1106 & 941 & 0.752 \\
\hline 29 & 0.2 & 30 & 205 & 66.5 & 0.325 & 167 & 33.5 & 0.200 & 658 & $X$ & 0.194 & 0.159 & 0 & 27.3 & 26.5 & 24.4 & 1248 & 1010 & 0.799 \\
\hline 30 & 0.2 & 50 & 205 & 66.6 & 0.326 & 168 & 33.6 & 0.200 & 661 & $X$ & 0.199 & 0.165 & 0 & 26.7 & 26.0 & 23.6 & 1381 & 1075 & 0.776 \\
\hline 31 & 0.4 & 1 & 308 & 132 & 0.429 & 292 & 117 & 0.400 & 626 & $X$ & 0.400 & 0.341 & 0 & 14.0 & 14.5 & 12.7 & 855 & 804 & 0.719 \\
\hline 32 & 0.4 & 2 & 271 & 119 & 0.439 & 240 & 95.9 & 0.400 & 629 & $X$ & 0.323 & 0.278 & 0 & 17.2 & 17.1 & 15.4 & 940 & 828 & 0.785 \\
\hline 33 & 0.4 & 4 & 246 & 112 & 0.454 & 213 & 85.4 & 0.400 & 635 & $X$ & 0.265 & 0.238 & 0 & 20.7 & 18.8 & 17.1 & 1081 & 883 & 0.861 \\
\hline 34 & 0.4 & 6 & 246 & 111 & 0.451 & 214 & 85.6 & 0.400 & 638 & $X$ & 0.262 & 0.235 & 0 & 20.9 & 20.1 & 18.5 & 1180 & 923 & 0.833 \\
\hline 35 & 0.4 & 8 & 241 & 110 & 0.456 & 208 & 83.3 & 0.400 & 644 & $\mathrm{X}$ & 0.248 & 0.226 & 0 & 22.0 & 21.0 & 19.4 & 1271 & 963 & 0.823 \\
\hline 36 & 0.4 & 10 & 232 & 104 & 0.447 & 199 & 76.4 & 0.384 & 652 & 398 & 0.233 & 0.217 & 0.608 & 23.2 & 22.2 & 19.8 & 1415 & 1008 & 0.679 \\
\hline 37 & 0.4 & 12 & 227 & 99.0 & 0.436 & 195 & 71.4 & 0.366 & 652 & 393 & 0.227 & 0.213 & 0.625 & 23.8 & 22.9 & 20.6 & 1457 & 1033 & 0.693 \\
\hline 38 & 0.4 & 15 & 221 & 92.5 & 0.420 & 188 & 64.6 & 0.343 & 655 & 386 & 0.213 & 0.204 & 0.629 & 25.1 & 23.9 & 21.7 & 1502 & 1050 & 0.624 \\
\hline 39 & 0.4 & 30 & 212 & 78.5 & 0.370 & 172 & 47.2 & 0.274 & 657 & 360 & 0.191 & 0.184 & 0.653 & 27.6 & 26.6 & 24.6 & 1622 & 1120 & 0.686 \\
\hline 40 & 0.4 & 50 & 207 & 70.5 & 0.341 & 175 & 42.0 & 0.240 & 661 & 341 & 0.191 & 0.180 & 0.673 & 27.6 & 26.6 & 24.5 & 1701 & 1183 & 0.706 \\
\hline 41 & 0.6 & 1 & 363 & 222 & 0.611 & 339 & 204 & 0.600 & 624 & 506 & 0.443 & 0.450 & 0.000 & 12.7 & 12.3 & 11.1 & 950 & 920 & 0.882 \\
\hline 42 & 0.6 & 2 & 339 & 205 & 0.605 & 311 & 185 & 0.594 & 624 & 523 & 0.346 & 0.414 & 0.060 & 16.1 & 13.3 & 12.4 & 1105 & 932 & 0.766 \\
\hline 43 & 0.6 & 4 & 270 & 154 & 0.572 & 241 & 134 & 0.556 & 635 & 507 & 0.295 & 0.306 & 0.510 & 18.7 & 17.5 & 16.0 & 1239 & 943 & 0.641 \\
\hline 44 & 0.6 & 6 & 261 & 134 & 0.514 & 225 & 110 & 0.488 & 641 & 478 & 0.269 & 0.273 & 0.613 & 20.4 & 18.9 & 17.2 & 1333 & 973 & 0.648 \\
\hline 45 & 0.6 & 8 & 247 & 120 & 0.484 & 214 & 95.4 & 0.446 & 647 & 440 & 0.252 & 0.249 & 0.630 & 21.6 & 20.1 & 17.8 & 1390 & 1000 & 0.651 \\
\hline 46 & 0.6 & 10 & 239 & 109 & 0.455 & 205 & 83.9 & 0.410 & 652 & 419 & 0.240 & 0.235 & 0.647 & 22.6 & 21.0 & 18.7 & 1437 & 1021 & 0.668 \\
\hline 47 & 0.6 & 12 & 231 & 100 & 0.433 & 197 & 74.5 & 0.378 & 652 & 397 & 0.230 & 0.223 & 0.668 & 23.5 & 21.8 & 19.6 & 1478 & 1042 & 0.704 \\
\hline 48 & 0.6 & 15 & 224 & 93.0 & 0.415 & 191 & 67.9 & 0.356 & 655 & 391 & 0.215 & 0.212 & 0.670 & 24.9 & 22.8 & 20.7 & 1514 & 1060 & 0.693 \\
\hline 49 & 0.6 & 30 & 214 & 77.0 & 0.360 & 173 & 48.7 & 0.282 & 657 & 350 & 0.190 & 0.187 & 0.669 & 27.8 & 26.1 & 24.1 & 1628 & 1127 & 0.668 \\
\hline 50 & 0.6 & 50 & 209 & 68.5 & 0.328 & 175 & 36.6 & 0.209 & 661 & 328 & 0.191 & 0.182 & 0.693 & 27.6 & 26.1 & 23.9 & 1704 & 1191 & 0.703 \\
\hline 51 & 0.8 & 1 & 437 & 327 & 0.747 & 408 & 309 & 0.757 & 618 & 562 & 0.544 & 0.662 & 0.227 & 10.4 & 9.70 & 8.46 & 1057 & 937 & 0.645 \\
\hline 52 & 0.8 & 2 & 350 & 238 & 0.681 & 319 & 217 & 0.678 & 621 & 528 & 0.413 & 0.465 & 0.474 & 13.6 & 12.4 & 11.2 & 1167 & 952 & 0.669 \\
\hline 53 & 0.8 & 4 & 295 & 176 & 0.598 & 265 & 158 & 0.598 & 624 & 476 & 0.320 & 0.356 & 0.626 & 17.4 & 16.1 & 14.4 & 1274 & 942 & 0.640 \\
\hline 54 & 0.8 & 6 & 264 & 136 & 0.514 & 231 & 116 & 0.501 & 630 & 449 & 0.276 & 0.291 & 0.649 & 19.9 & 18.4 & 16.6 & 1350 & 984 & 0.649 \\
\hline 55 & 0.8 & 8 & 252 & 122 & 0.483 & 219 & 100 & 0.458 & 640 & 423 & 0.257 & 0.267 & 0.653 & 21.3 & 19.6 & 17.8 & 1402 & 1010 & 0.652 \\
\hline 56 & 0.8 & 10 & 243 & 112 & 0.461 & 211 & 88.9 & 0.422 & 642 & 391 & 0.245 & 0.247 & 0.663 & 22.2 & 20.4 & 18.4 & 1447 & 1033 & 0.664 \\
\hline 57 & 0.8 & 12 & 236 & 102 & 0.432 & 204 & 81.0 & 0.397 & 647 & 376 & 0.234 & 0.233 & 0.671 & 23.1 & 21.0 & 19.2 & 1483 & 1052 & 0.682 \\
\hline 58 & 0.8 & 15 & 230 & 93.0 & 0.405 & 192 & 69.9 & 0.364 & 650 & 362 & 0.216 & 0.219 & 0.674 & 24.8 & 22.8 & 20.7 & 1517 & 1062 & 0.694 \\
\hline 59 & 0.8 & 30 & 215 & 76.0 & 0.353 & 173 & 49.1 & 0.283 & 658 & 334 & 0.200 & 0.175 & 0.678 & 26.6 & 25.7 & 23.6 & 1623 & 1130 & 0.610 \\
\hline 60 & 0.8 & 50 & 212 & 70.4 & 0.333 & 174 & 42.6 & 0.245 & 658 & 315 & 0.194 & 0.168 & 0.637 & 27.3 & 25.4 & 23.5 & 1695 & 1130 & 0.658 \\
\hline 61 & 1.0 & 1 & 478 & 389 & 0.812 & 443 & 367 & 0.829 & 606 & 517 & 0.609 & 0.721 & 0.463 & 9.32 & 9.46 & 8.25 & 1089 & 977 & 0.623 \\
\hline 62 & 1.0 & 2 & 365 & 269 & 0.736 & 335 & 249 & 0.744 & 612 & 502 & 0.437 & 0.563 & 0.497 & 12.9 & 12.2 & 11.0 & 1202 & 970 & 0.636 \\
\hline 63 & 1.0 & 4 & 305 & 210 & 0.689 & 272 & 169 & 0.623 & 629 & 480 & 0.333 & 0.385 & 0.629 & 16.7 & 16.0 & 14.3 & 1288 & 947 & 0.642 \\
\hline 64 & 1.0 & 6 & 268 & 141 & 0.526 & 231 & 116 & 0.501 & 635 & 454 & 0.283 & 0.306 & 0.624 & 19.5 & 18.4 & 16.9 & 1356 & 988 & 0.647 \\
\hline 65 & 1.0 & 8 & 254 & 123 & 0.484 & 221 & 104 & 0.470 & 641 & 447 & 0.260 & 0.277 & 0.663 & 21.0 & 19.7 & 17.7 & 1410 & 1010 & 0.646 \\
\hline 66 & 1.0 & 10 & 245 & 111 & 0.453 & 213 & 91.2 & 0.428 & 647 & 402 & 0.247 & 0.251 & 0.673 & 22.0 & 20.5 & 18.5 & 1455 & 1036 & 0.669 \\
\hline 67 & 1.0 & 12 & 238 & 102 & 0.429 & 206 & 82.8 & 0.402 & 652 & 390 & 0.236 & 0.236 & 0.695 & 23.0 & 21.3 & 19.5 & 1489 & 1054 & 0.695 \\
\hline 68 & 1.0 & 15 & 228 & 91.0 & 0.399 & 191 & 69.9 & 0.366 & 652 & 378 & 0.219 & 0.220 & 0.702 & 24.5 & 23.0 & 21.1 & 1530 & 1068 & 0.700 \\
\hline 69 & 1.0 & 30 & 217 & 72.5 & 0.334 & 180 & 52.5 & 0.292 & 655 & 341 & 0.190 & 0.200 & 0.710 & 27.8 & 25.1 & 22.7 & 1628 & 1128 & 0.630 \\
\hline 70 & 1.0 & 50 & 212 & 70.2 & 0.331 & 173 & 44.7 & 0.259 & 658 & 326 & 0.191 & 0.191 & 0.686 & 27.6 & 26.3 & 24.6 & 1694 & 1128 & 0.654 \\
\hline
\end{tabular}


Table 5

The tool edge radius is $R=0.03 \mathrm{~mm}$, while we have $R=0.015 \mathrm{~mm}$ in other tables.

\begin{tabular}{|c|c|c|c|c|c|c|c|c|c|c|c|c|c|c|c|c|c|c|c|}
\hline \multicolumn{20}{|c|}{$t_{1}=0.1 \mathrm{~mm}, \mu=0.8, \kappa=2000 \mathrm{Wm}^{-2} \mathrm{~K}^{-1}, \beta=0.9$} \\
\hline \# & $\begin{array}{l}R \\
(\mathrm{~mm})\end{array}$ & $V(\mathrm{~m} / \mathrm{s})$ & $F_{C}(\mathrm{kN} / \mathrm{m})$ & $F_{T}(\mathrm{kN} / \mathrm{m})$ & $\mu_{a p}$ & $\begin{array}{l}F_{n}(\text { rake }) \\
(\mathrm{kN} / \mathrm{m})\end{array}$ & $\begin{array}{l}F_{t}(\text { rake }) \\
(\mathrm{kN} / \mathrm{m})\end{array}$ & $\bar{\mu}$ & $\begin{array}{l}\bar{\tau}_{P Z} \\
(\mathrm{MPa})\end{array}$ & $\begin{array}{l}\bar{\tau}_{S Z} \\
(\mathrm{MPa})\end{array}$ & $\begin{array}{l}t_{2} \\
(\mathrm{~mm})\end{array}$ & $\begin{array}{l}l_{c} \\
(\mathrm{~mm})\end{array}$ & $l_{p} / l_{c}$ & $\begin{array}{l}\phi \\
\text { (deg.) }\end{array}$ & $\begin{array}{l}\phi_{A} \\
\text { (deg.) }\end{array}$ & $\begin{array}{l}\phi^{*} \\
\text { (deg.) }\end{array}$ & $\begin{array}{l}T_{\max } \\
(\mathrm{K})\end{array}$ & $\begin{array}{l}T_{1} \\
(\mathrm{~K})\end{array}$ & $\begin{array}{l}X_{\max } / \\
l_{c}\end{array}$ \\
\hline R01 & 0.03 & 4 & 288 & 182 & 0.632 & 227 & 133 & 0.586 & 623 & 491 & 0.295 & 0.312 & 0.503 & 18.7 & 17.0 & 13.4 & 1249 & 967 & 0.587 \\
\hline R02 & 0.03 & 6 & 268 & 157 & 0.586 & 214 & 111 & 0.519 & 629 & 451 & 0.267 & 0.276 & 0.518 & 20.5 & 18.5 & 14.4 & 1328 & 1012 & 0.567 \\
\hline R03 & 0.03 & 10 & 249 & 133 & 0.533 & 189 & 83.0 & 0.439 & 645 & 400 & 0.239 & 0.237 & 0.565 & 22.7 & 19.9 & 15.9 & 1437 & 1080 & 0.613 \\
\hline R04 & 0.03 & 15 & 239 & 119 & 0.498 & 180 & 70.0 & 0.387 & 647 & 364 & 0.226 & 0.215 & 0.605 & 23.9 & 20.7 & 16.6 & 1519 & 1142 & 0.658 \\
\hline R05 & 0.03 & 30 & 227 & 104 & 0.456 & 168 & 53.8 & 0.321 & 651 & 304 & 0.207 & 0.194 & 0.608 & 25.8 & 22.4 & 18.1 & 1626 & 1244 & 0.671 \\
\hline
\end{tabular}

Table 6

Variation of the uncut chip thickness $t_{1}$.

\begin{tabular}{|c|c|c|c|c|c|c|c|c|c|c|c|c|c|c|c|c|c|c|c|}
\hline \# & $\begin{array}{l}t_{1} \\
(\mathrm{~mm})\end{array}$ & $V(\mathrm{~m} / \mathrm{s})$ & $F_{C}(\mathrm{kN} / \mathrm{m})$ & $F_{T}(\mathrm{kN} / \mathrm{m})$ & $\mu_{a p}$ & $\begin{array}{l}F_{n}(\text { rake }) \\
(\mathrm{kN} / \mathrm{m})\end{array}$ & $\begin{array}{l}F_{t}(\text { rake }) \\
(\mathrm{kN} / \mathrm{m})\end{array}$ & $\bar{\mu}$ & $\begin{array}{l}\bar{\tau}_{P Z} \\
(\mathrm{MPa})\end{array}$ & $\begin{array}{l}\bar{\tau}_{S Z} \\
(\mathrm{MPa})\end{array}$ & $\begin{array}{l}t_{2} \\
(\mathrm{~mm})\end{array}$ & $\begin{array}{l}l_{c} \\
(\mathrm{~mm})\end{array}$ & $l_{p} / l_{c}$ & $\begin{array}{l}\phi \\
\text { (deg.) }\end{array}$ & $\begin{array}{l}\phi_{A} \\
\text { (deg.) }\end{array}$ & $\begin{array}{l}\phi^{*} \\
\text { (deg.) }\end{array}$ & $\begin{array}{l}T_{\max } \\
(\mathrm{K})\end{array}$ & $\begin{array}{l}T_{1} \\
(\mathrm{~K})\end{array}$ & $\begin{array}{l}X_{\max } l \\
l_{c}\end{array}$ \\
\hline T01 & 0.05 & 4 & 173 & 110 & 0.636 & 143 & 86.7 & 0.604 & 643 & $X$ & 0.182 & 0.185 & 0 & 15.4 & 13.7 & 10.6 & 1043 & 865 & 0.730 \\
\hline T02 & 0.05 & 8 & 158 & 96.2 & 0.609 & 124 & 69.7 & 0.562 & 656 & 551 & 0.153 & 0.160 & 0.413 & 18.1 & 16.1 & 12.5 & 1176 & 891 & 0.625 \\
\hline T03 & 0.05 & 20 & 151 & 78.3 & 0.518 & 119 & 53.2 & 0.447 & 687 & 440 & 0.141 & 0.143 & 0.566 & 19.5 & 16.9 & 13.2 & 1379 & 993 & 0.622 \\
\hline T04 & 0.05 & 40 & 145 & 67.7 & 0.467 & 114 & 42.3 & 0.371 & 704 & 387 & 0.130 & 0.132 & 0.576 & 21.0 & 17.7 & 14.3 & 1505 & 1066 & 0.667 \\
\hline T05 & 0.15 & 4 & 367 & 182 & 0.496 & 333 & 158 & 0.474 & 631 & 463 & 0.380 & 0.381 & 0.654 & 21.5 & 20.2 & 18.8 & 1330 & 958 & 0.677 \\
\hline T06 & 0.15 & 8 & 327 & 138 & 0.422 & 294 & 111 & 0.380 & 641 & 393 & 0.324 & 0.318 & 0.695 & 24.8 & 23.2 & 21.9 & 1475 & 1022 & 0.711 \\
\hline T07 & 0.15 & 20 & 306 & 105 & 0.343 & 273 & 78.1 & 0.286 & 651 & 305 & 0.298 & 0.287 & 0.704 & 26.7 & 24.7 & 23.2 & 1621 & 1118 & 0.739 \\
\hline T08 & 0.15 & 40 & 294 & 87.9 & 0.299 & 256 & 58.9 & 0.230 & 656 & 242 & 0.277 & 0.269 & 0.699 & 28.4 & 26.9 & 25.4 & 1705 & 1185 & 0.213 \\
\hline T09 & 0.25 & 4 & 554 & 234 & 0.422 & 516 & 209 & 0.405 & 630 & 425 & 0.589 & 0.560 & 0.725 & 23.0 & 21.6 & 20.7 & 1440 & 1010 & 0.743 \\
\hline T10 & 0.25 & 8 & 500 & 172 & 0.344 & 475 & 150 & 0.316 & 647 & 349 & 0.490 & 0.470 & 0.745 & 27.0 & 25.9 & 25.0 & 1559 & 1045 & 0.770 \\
\hline T11 & 0.25 & 20 & 471 & 126.0 & 0.268 & 436 & 101 & 0.232 & 652 & 250 & 0.465 & 0.437 & 0.767 & 28.3 & 27.3 & 26.5 & 1680 & 1140 & 0.787 \\
\hline T12 & 0.25 & 40 & 455 & 102.0 & 0.224 & 432 & 80.7 & 0.187 & 658 & 204 & 0.427 & 0.406 & 0.756 & 30.3 & 29.8 & 28.6 & 1748 & 1204 & 0.781 \\
\hline T13 & 0.50 & 4 & 981 & 324 & 0.330 & 942 & 298 & 0.316 & 641 & 338 & 1.015 & 0.949 & 0.813 & 26.2 & 24.7 & 24.3 & 1563 & 1035 & 0.819 \\
\hline T14 & 0.50 & 8 & 920 & 240 & 0.261 & 883 & 212 & 0.240 & 647 & 271 & 0.908 & 0.849 & 0.820 & 28.8 & 27.7 & 27.2 & 1648 & 1072 & 0.826 \\
\hline T15 & 0.50 & 20 & 879 & 168 & 0.191 & 843 & 141 & 0.167 & 654 & 189 & 0.889 & 0.802 & 0.832 & 29.4 & 28.1 & 27.7 & 1731 & 1134 & 0.838 \\
\hline T16 & 0.50 & 40 & 854 & 130 & 0.152 & 813 & 102 & 0.125 & 655 & 145 & 0.830 & 0.750 & 0.837 & 31.1 & 30.2 & 29.8 & 1782 & 1216 & 0.850 \\
\hline
\end{tabular}

Thus, in general $\mu_{a p}$ is function of $\mu$, the material properties of the work-material (essentially through the mean value $\bar{\tau}_{S Z}$ of the shear flow stress along the sticking zone), the tool edge radius $R$ and the cutting conditions (cutting speed, rake angle, feed).

$\mu_{a p}=\mu_{a p}\left(\mu, \bar{\tau}_{S Z}, R\right.$, cutting conditions $)$

Note that the cutting conditions are also indirectly appearing through $\bar{\tau}_{S Z}$.

It is also useful to introduce the mean friction coefficient $\bar{\mu}$ along the tool rake face. This parameter does not include explicitly the contribution of the contact forces exerted on the rounded tool edge. Rather $\bar{\mu}$ characterizes the contact along the flat part of the tool rake-face (region IJ in Fig. 1b) and is defined as

$\bar{\mu}=\frac{F_{t}(\text { rake })}{F_{n}(\text { rake })}$

$F_{t}$ (rake) and $F_{n}$ (rake) are, respectively, the tangential and the normal components of the force (per unit cutting width) exerted by the chip onto IJ:

$F_{n}($ rake $)=\int_{0}^{l_{c}} \sigma d x \quad F_{t}($ rake $)=\int_{0}^{l_{p}} \tau_{Y} d x+\mu \int_{l_{p}}^{l_{c}} \sigma d x$

where $\tau_{Y}$ is the shear flow stress. The values of $F_{n}($ rake $)$ and $F_{t}($ rake $)$ are reported in Tables $4-6$. In the following, $\bar{\mu}$ is denoted as the mean friction coefficient.

The distinction between the friction characteristic $\bar{\mu}$ of the flat rake face and the global friction $\mu_{a p}$ was discussed by Albrecht [23].
Combining (7) and (8) yields:

$\bar{\mu}=\mu-\frac{D}{F_{n}(\text { rake })}$ with $D=\int_{0}^{l_{p}}\left(\mu \sigma-\tau_{Y}\right) d x$

Since $\tau_{Y} \leq \mu \sigma$ along the sticking zone, $D$ is positive and

$\bar{\mu} \leq \mu$

The equality

$\bar{\mu}=\mu$

is only satisfied when there is no sticking, i.e. $l_{p}=0$.

In general $\mu_{a p}>\bar{\mu}$, except when the tool is perfectly sharp. All friction coefficients defined above are identical, $\mu_{a p}=\bar{\mu}=\mu$, if the following conditions are met: (i) there is no sticking, (ii) the tool is perfectly sharp, and (iii) there is no clearance contact.

A constitutive law similar to that for $\mu_{a p}$, Eq. (6), is expected to hold for $\bar{\mu}$, however with a weaker dependence with respect to the tool edge radius.

\section{Factors governing the evolution of the mean friction coefficient}

\subsection{Role of sticking contact and of thermal softening}

The dependence of the mean friction coefficient $\bar{\mu}$ upon the sliding friction coefficient $\mu$ and the cutting speed $V$ is illustrated in Fig. 3. Cutting conditions are those of Table 4 . For $\mu=0$ and 0.2 there is no sticking along the rake face and $\bar{\mu}=\mu$. For $\mu \geq 0.4, \bar{\mu}$ appears to be a decreasing function of $V$. The weakening of $\bar{\mu}$ with velocity 


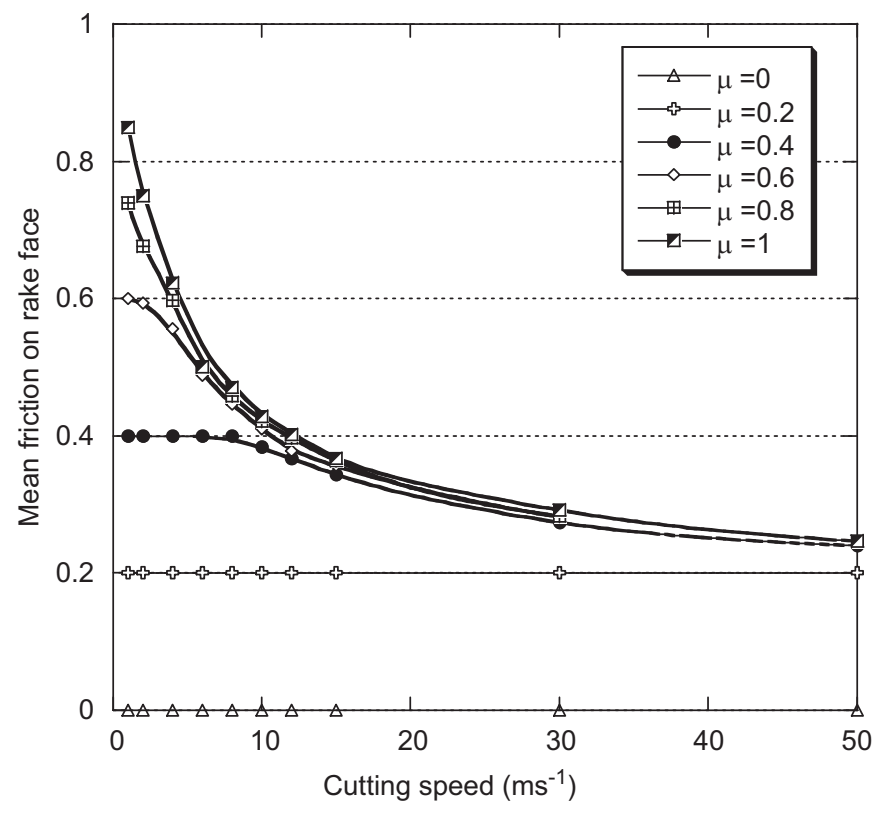

Fig. 3. Effect of the cutting speed $V$ on the mean friction coefficient $\bar{\mu}$ (accounting for the contact forces exerted on the tool rake face IJ, see Fig. 1b). Various values of the sliding friction coefficient $\mu$ are considered. Note that $\bar{\mu}=\mu$ for small values of $\mu$ since sticking does not occur in that case. For large values of $\mu$, it appears that $\bar{\mu}<\mu$ and that $\bar{\mu}$ is a decreasing function of $V$ as a result of thermal softening of the workmaterial along the secondary shear zone. For $\mu=0.4$ and 0.6 it is observed that $\bar{\mu}$ is tending to $\mu$ for small values of the cutting speed, as sliding contact is dominant.

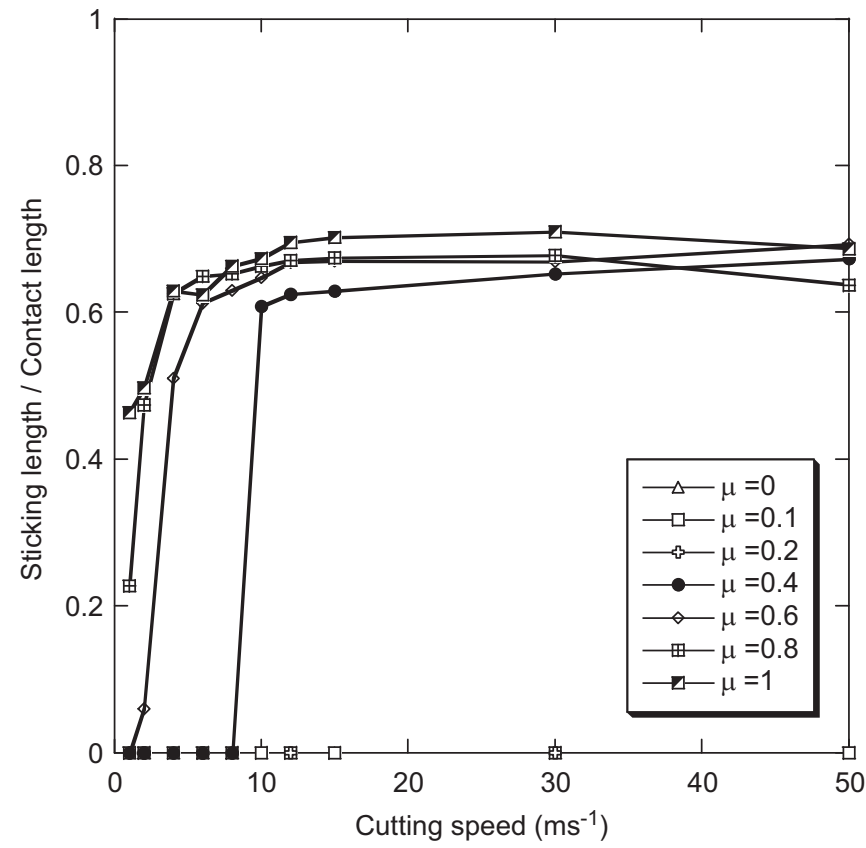

Fig. 4. Evolution of the sticking ratio $l_{p} / l_{c}$ with the cutting speed; $l_{p}$ is the sticking length and $l_{c}$ is the tool-chip contact length. For low values of the sliding friction coefficient $\mu$, there is no sticking along the rake face $\left(l_{p}=0\right)$. For $\mu \geq 0.4$ a transition towards a contact regime dominated by sticking is apparent when the cutting speed is increased.

(velocity softening) appears only when sticking contact is activated along the rake face. This is clearly demonstrated by comparing Fig. 3 with Fig. 4 that shows the evolution of the sticking ratio $l_{p} / l_{c}$ in terms of the cutting speed for various values of $\mu$. A transition towards a contact regime dominated by sticking is observed with the sticking length $l_{p}$ being about $60 \%$ of the contact length. The occurrence of sticking is a consequence of the drop of the flow stress of the work material due to heating along the tool-chip interface. The increasing of the interface temperature results from two sources: (i) frictional heating along the sliding zone and (ii) conversion of the plastic work into heat along the sticking zone (within the so called secondary shear zone). The correlation between the softening of $\bar{\tau}_{S Z}$ and the interface heating can be seen by comparing Fig. $5 a$ with $5 b$ where the evolution of the maximal
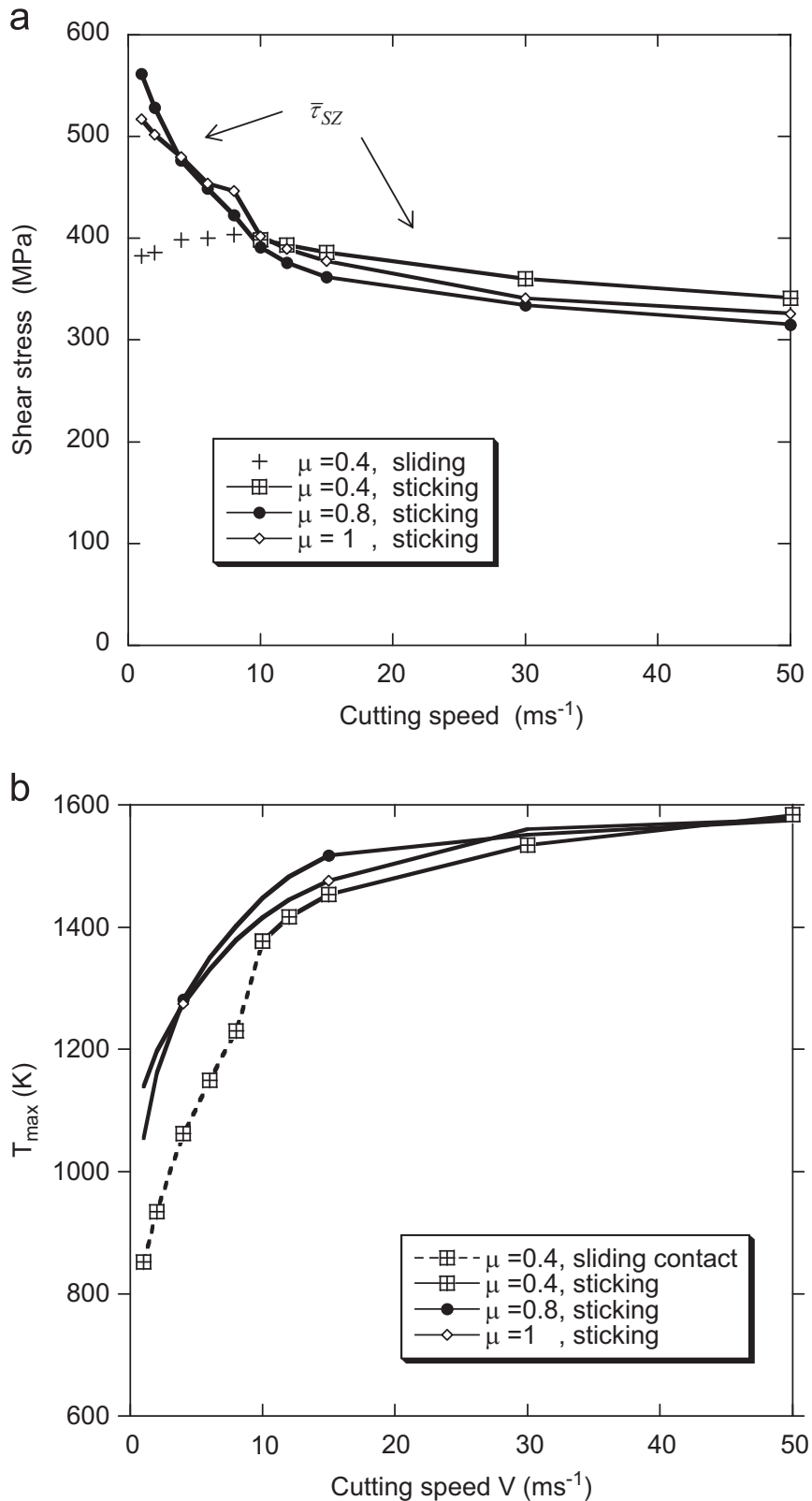

Fig. 5. (a) Variation with respect to the cutting speed $V$ of the mean shear flow stress $\bar{\tau}_{S Z}$ of the work-material along the sticking zone for large values of the sliding friction coefficient $\mu$. For $\mu=0.4$, contact is governed by sliding when $V \leq 8 \mathrm{~ms}^{-1}$. Crosses represent the value of the shear stress at the point $I$ of the rake face, see Fig. $1 \mathrm{~b}$. This stress is given by the Coulomb friction law under sliding condition. For $\mu=0.4$, sticking contact is activated if $V>8 \mathrm{~ms}^{-1}$. The solid line with crossed squares represents the evolution of $\bar{\tau}_{S Z}$. For $\mu=0.8$ and 1 , sticking happens at all values of $V$ considered here. Curves with black circles and white diamonds represent the evolution of $\bar{\tau}_{S Z}$. It is worth noting that $\bar{\tau}_{S Z}$ appears to be weakly dependent upon $\mu$ when contact is dominated by sticking. (b) Velocity dependence of the maximal temperature $T_{\max }$ of the work material at the tool-chip interface. Note that the velocity softening of $\bar{\mu}$ observed in (a) happens to be correlated to the increasing of temperature in the range of cutting speeds $0<V<15 \mathrm{~ms}^{-1}$. The saturation of $\bar{\tau}_{S Z}$ at large cutting speeds corresponds to the saturation of $T_{\max }$. 
temperature $T_{\max }$ of the work material at the tool-chip interface is displayed. These results are in agreement with the well known feature mentioned in the literature that the stress level at the rake face is controlled by friction heating at large enough cutting speed, see for instance [12].

The correlation between velocity softening of $\bar{\mu}$ and thermal softening of the flow stress can be further illustrated by observing in the Fig. 3 that the decay of $\bar{\mu}$ arises in the same velocity range $0 \leq V \leq 10 \mathrm{~ms}^{-1}$ than the drop of the mean flow stress along the sticking zone $\bar{\tau}_{S Z}$ depicted in Fig. $5 \mathrm{a}$.

It is worth noticing that for $\mu \geq 0.4$ the evolution of $\bar{\mu}$ reaches the same asymptotic regime at large cutting speeds, independently from the value of $\mu$. This asymptotic regime is related to the transition to a sticking dominated contact. Then, $\bar{\mu}$ appears to be mostly controlled by the mean shear flow-stress $\bar{\tau}_{S Z}$ along the sticking contact. Consequently, $\bar{\mu}$ becomes weakly dependent upon $\mu$. According to Fig. 4, the transition to a sticking dominated contact is achieved for $V \geq 8 \mathrm{~ms}^{-1}$ when $\mu \geq 0.4$. These conditions are exactly those for which all values of $\bar{\mu}$ are merging in the Fig. 3. Note that for $\mu=0.4$, there is no sticking for $V \leq 8 \mathrm{~ms}^{-1}$, therefore $\bar{\mu}=\mu$ in this velocity range. The same situation occurs for $\mu=0.6$ but only for $V \leq 2 \mathrm{~ms}^{-1}$.

For a given value $\mu \geq 0.4$ of the sliding friction coefficient, the existence of an asymptotic regime (with a low value of $\bar{\mu}$ that is independent of the cutting speed) is related to the emergence of a boundary layer regime along the sticking zone at the tool-chip interface. This is in keeping with the remark made by Childs [12] that "at speeds greater than tens of $\mathrm{m} / \mathrm{min}$, for steels, local thermal softening of chip material due to friction heating results in a degree of self-lubrication".

Boundary layer regime has been analyzed in the general context of thermo-viscoplastic materials by Gioia and Ortiz [24] and will be the object of a subsequent work in the case of machining.

\subsection{Effect of the conversion of plastic work into heat}

As discussed before, the weakening of $\bar{\mu}$ with velocity is due to the conversion of the plastic work into heat and the resulting thermal softening of the work-material (decay of $\bar{\tau}_{S Z}$ ).

The rate of conversion of plastic work into heat is controlled by the Taylor-Quinney coefficient $\beta$, see Eq. (2). The effect of the thermo-mechanical coupling parameter $\beta$ is analyzed by performing a parametric analysis for $t_{1}=0.1 \mathrm{~mm}, R=0.015 \mathrm{~mm}$, $\kappa=2000 \mathrm{Wm}^{-2} \mathrm{~K}^{-1}$ and $\mu=0.6$. Fig. 6 shows the variation of $\bar{\mu}$ in terms of the cutting speed $V$ for various values of $\beta$. As expected, for a given value of the cutting speed, $\bar{\mu}$ is a decreasing function of $\beta$ as a consequence of the larger amount of plastic work converted into heat. For a given $\beta$, it appears that $\bar{\mu}$ is tending to $\mu$ when $V$ decreases to zero. In fact, at low cutting speed, the interface temperature is cooled down by heat transfer effects. Thus, thermal softening of the shear flow stress is reduced and sticking contact is less likely to occur. Consequently, for $\mu=0.6$, sliding is the dominant mode of contact and $\bar{\mu} \approx \mu$. Similarly, for a given value of $V$ sliding contact is favoured when $\beta$ is decreased. This is why $\bar{\mu} \approx \mu$ in Fig. 6 for $V \leq 10 \mathrm{~ms}^{-1}$ and $\beta=0.5$. At the limiting case $\beta=0$ (not shown in Fig. 6) there would be no conversion of plastic work into heat and the thermal softening of the mean flow stress $\bar{\tau}_{S Z}$ along the sticking contact could only be the result of the heat generated by friction. However, frictional heat originates from the sliding contact zone and is convected away by the chip flow. Hence, frictional heating is not expected to affect the temperature in the secondary shear zone. That is why $\bar{\mu}$ could not weaken with the cutting speed for $\beta=0$.

In Fig. 3 it is observed that, for $\mu \geq 0.4$, the mean friction coefficient $\bar{\mu}$ becomes independent of the value of $\mu$ at large cutting speeds. On the contrary, a strong dependence of $\bar{\mu}$ upon the coefficient $\beta$ is apparent in Fig. 6 at large enough values of $V$.

It is known that $\beta$ can be rate dependent in certain conditions, as shown by the experiments of Rittel et al. [25] and Ravichandran et al. [26]. Therefore, an additional velocity dependence of $\bar{\mu}$ could be expected through rate effects embodied in $\beta$. These effects are not accounted for here, since it was assumed that $\beta$ is independent of cutting conditions. In fact, the experiments reported in $[25,26]$ indicate that the maximum value of $\beta$ (close to unity) is observed at strain rates larger than $10^{4} \mathrm{~s}^{-1}$. This range of strain rates is encountered in the primary and secondary shear zones during simulations. Thus, having considered in the modeling the fixed value $\beta=0.9$ appears to be a reasonable assumption. This assumption might be revised at cutting speeds lower than $V=1 \mathrm{~ms}^{-1}$.

\subsection{Evolution law for the mean friction coefficient}

In-depth analysis of the factors contributing to the decay of $\bar{\mu}$ with $V$ can be conducted by analytical means for large enough values of $\mu$ when sticking contact is significant. The tangential and normal forces (resp. $F_{t}\left(\right.$ rake) and $F_{n}($ rake $)$ ) exerted by the chip on the tool rake face can be evaluated by considering that the distribution of the normal stress along the tool rake face is of the form:

$\sigma(x)=\sigma_{0}\left(1-\frac{x}{l_{c}}\right)^{\xi}$

$x=0$ corresponds to the limit $I$ of the tool rake face, see Fig. $1 \mathrm{~b}$. The stress exponent $\xi$ controls the stress decay when $x$ increases. The stress vanishes at the end of the contact $\left(x=l_{c}\right)$. In the following, a value of $\xi$ will be associated to a given stress profile.

The tangential force $F_{t}$ (rake) (per unit cutting width) can be evaluated with use of Eq. (8b) and Eq. (11):

$F_{t}($ rake $)=l_{p} \bar{\tau}_{S Z}+\frac{\mu \sigma_{0} l_{c}}{\xi+1}\left(1-\frac{l_{p}}{l_{c}}\right)^{\xi+1}$

The shear stress at the limit of the sliding zone $\left(x=l_{p}\right)$ is obtained with the Coulomb friction law and Eq. (11): $\tau^{*}=\mu \sigma_{0}\left(1-\left(l_{p} / l_{c}\right)\right)^{\xi}$. The stress profiles obtained numerically show a smooth variation of the shear stress at $x=l_{p}$ (while the normal stress can vary more abruptly). Thus, the shear stress at $x=l_{p}$ is close to the mean value $\bar{\tau}_{S Z}$ (over the sticking zone) and it can be stated that $\tau^{*}=\omega \bar{\tau}_{S Z}$, where $\omega$ is a scaling factor close to unity $(0<\omega<1)$. From this condition it follows that:

$\frac{l_{p}}{l_{c}}=1-\left(\frac{\omega \bar{\tau}_{S Z}}{\mu \sigma_{0}}\right)^{1 / \xi}$

When $\omega \bar{\tau}_{S Z} \geq \mu \sigma_{0}$, there is no sticking and consequently $l_{p} / l_{c}=0$. Altogether, it can be written that:

$\frac{l_{p}}{l_{c}}=\max \left(0,1-\left(\frac{\omega \bar{\tau}_{S Z}}{\mu \sigma_{0}}\right)^{1 / \xi}\right)$

Using (13) together with (12) gives:

$F_{t}($ rake $)=\frac{l_{c} \bar{\tau}_{S Z}}{\xi+1}\left(\omega+\frac{l_{p}}{l_{c}}(\xi+1-\omega)\right)$

Finally, the mean friction can be evaluated as

$\bar{\mu}=\frac{F_{t}(\text { rake })}{F_{n}(\text { rake })}=\frac{l_{c} \bar{\tau}_{S Z}}{F_{n}(\text { rake })} \frac{1}{(\xi+1)}\left(\omega+\frac{l_{p}}{l_{c}}(\xi+1-\omega)\right)$ for $l_{p}>0$

The normal force is

$F_{n}($ rake $)=\int_{0}^{l_{c}} \sigma d x=\frac{\sigma_{0} l_{c}}{\xi+1}$ 
After substitution into (15) it follows that:

$\bar{\mu}=\frac{\bar{\tau}_{S Z}}{\sigma_{0}}\left(\omega+\frac{l_{p}}{l_{c}}(\xi+1-\omega)\right)$

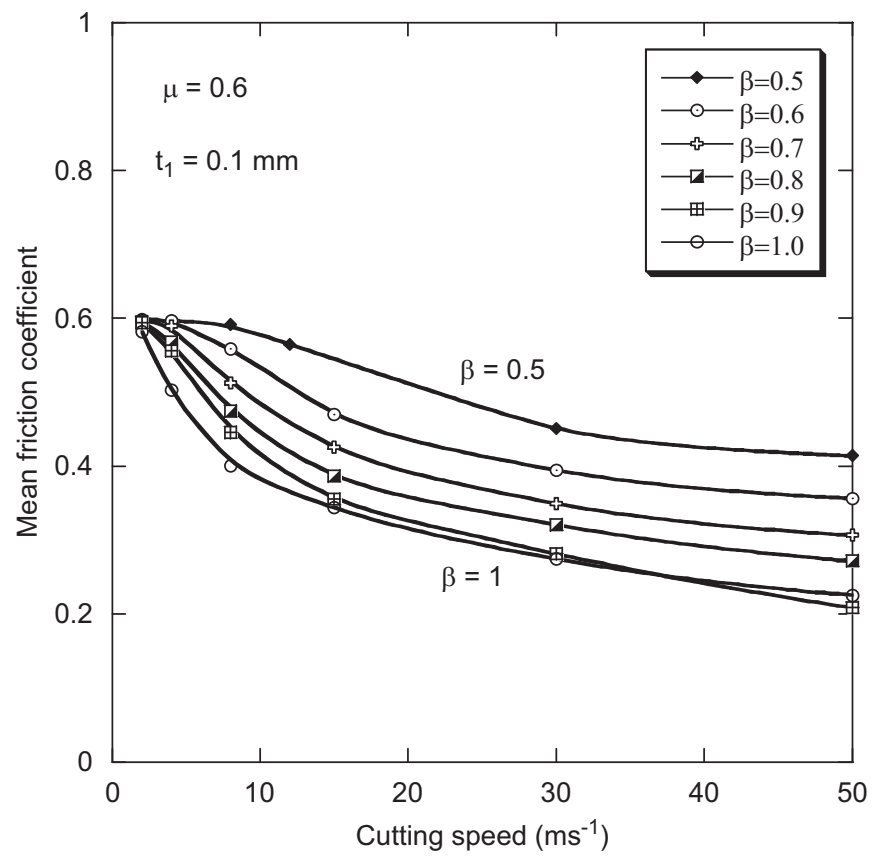

Fig. 6. Effect on the mean friction coefficient $\bar{\mu}$ of the coefficient $\beta$ of convertion of plastic work into heat. Cutting conditions are: $t_{1}=0.1 \mathrm{~mm}, R=0.015 \mathrm{~mm}$, and the sliding friction coefficient is $\mu=0.6$.
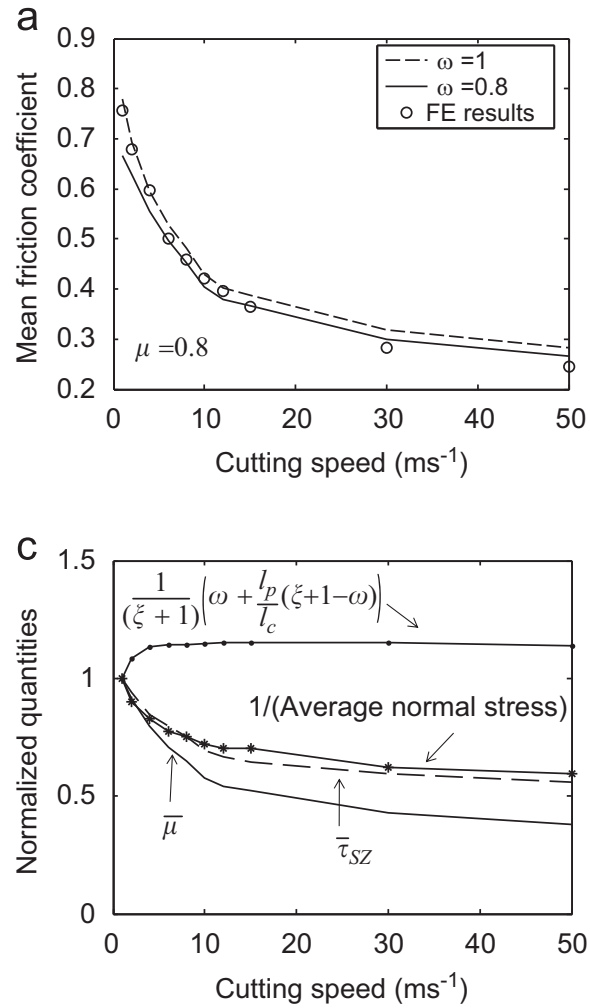

Another useful expression for $\bar{\mu}$ is obtained by combining Eq. (16) and Eq. (13) with Eq. (15):

$\bar{\mu}=\frac{l_{c} \bar{\tau}_{S Z}}{F_{n}(\text { rake })} \frac{1}{(\xi+1)}\left(\omega+(\xi+1-\omega)\left(1-\left(\frac{\omega l_{c} \bar{\tau}_{S Z}}{\mu(\xi+1)\left|F_{Y}\right|}\right)^{1 / \xi}\right)\right)$

From (15) $\bar{\mu}$ appears essentially as the ratio of two terms: (i) the mean shear stress $\bar{\tau}_{S Z}$ over the sticking zone (weighted by the factor $\left.(1 /(\xi+1))\left(\omega+\left(l_{p} / l_{c}\right)(\xi+1-\omega)\right)\right)$ and (ii) the average normal stress over the contact length $F_{n}($ rake $) / l_{c}$.

Fig. 7a shows for $\mu=0.8$ the evolution of $\bar{\mu}$ with respect to $V$ as predicted by Eq. (15) where the values of $\bar{\tau}_{S Z}, F_{n}$ (rake) $/ l_{c}$ and $l_{p} / l_{c}$ are taken from Table 4 . The stress exponent is taken to be $\xi=0.23$, a value which has been identified from stress profiles as shown in Appendix B. Two values of $\omega$ close to unity are considered, $\omega=1$ and 0.8. The consistency of the results derived with Eq. (15) is checked against values of $\bar{\mu}$ obtained directly from the Finite Element simulations reported in Table 4 . The value $\omega=0.9$ is adopted in the following, keeping in mind that the results are similar for any value of $\omega$ close to unity (which is the case when sticking is dominant).

In Fig. 7b Finite Element results are compared to the estimates of $\bar{\mu}$ obtained with Eqs. (15) and (18) for $\xi=0.23$ and $\omega=0.9$. Both relationships predict correct trends for the variation of $\bar{\mu}$ in terms of $V$, albeit with a slight overestimation in the case of Eq. (18).

Interest of using Eqs. (15) and (18) to assess the evolution of $\bar{\mu}$ with velocity stems from the possibility of evaluating the contributions of the different physical factors involved in the velocity weakening of $\bar{\mu}$. In Fig. 7c is represented for $\xi=0.23$ and $\omega=0.9$ the evolution of $\bar{\mu}$ with $V$ according to Eq. (15), together with the evolution of parameters $A=\bar{\tau}_{S Z}, B=\left(l_{c} / F_{n}(\right.$ rake $\left.)\right)$, and $C=\omega+\left(l_{p} / l_{c}\right)$ $(\xi+1-\omega)$, which are factors in expression (15) of $\bar{\mu}$. The values of
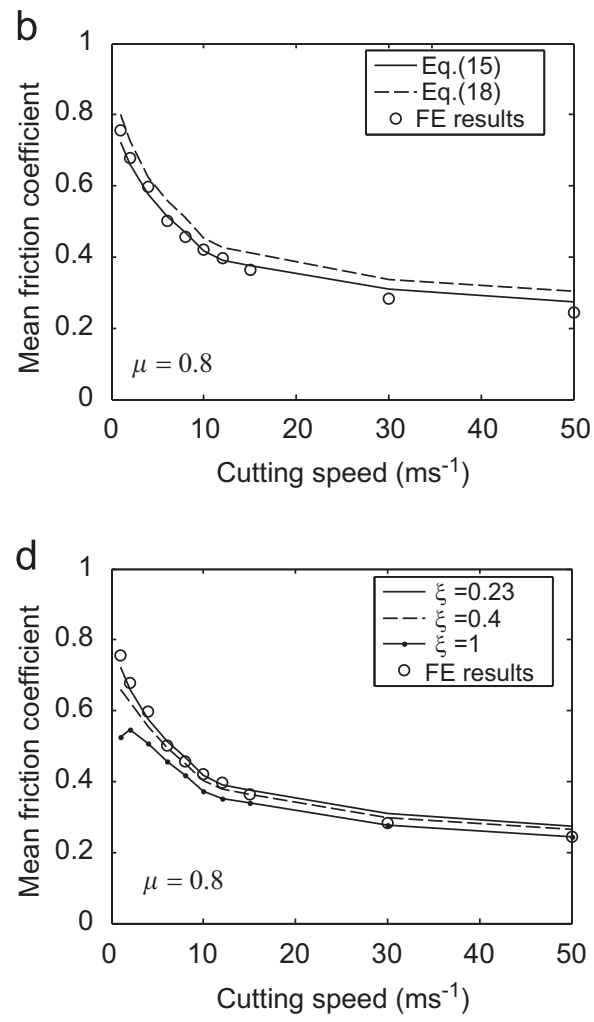

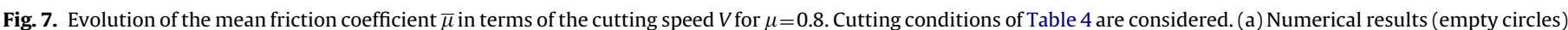

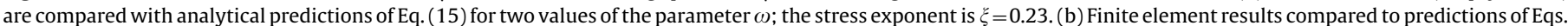

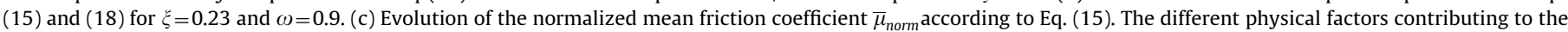

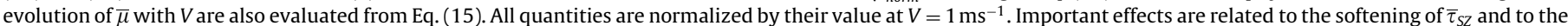
average normal stress. (d) Comparison of Finite Element results with predictions of Eq. (15) and evaluation of the effect of the stress exponent $\xi$ for $\omega=0.9$. 
these parameters in terms of $V$ are obtained from data reported in Table 4. It should be noted that the quantities displayed in Fig. 7c are normalized with respect to their value at the smallest speed considered $\left(V=1 \mathrm{~ms}^{-1}\right)$. Thus, in Fig. 7c are represented the variations of the normalized quantities $A_{\text {norm }}=A / A(V=1 \mathrm{~m} / \mathrm{s})$, $\bar{\mu}_{\text {norm }}, B_{\text {norm }}$ and $C_{\text {norm }}$. Since $\bar{\mu}_{\text {norm }}=A_{\text {norm }} B_{\text {norm }} C_{\text {norm }}$, each factor represents truly the relative contribution of a given parameter $A, B$, or $C$ to the variation of the mean friction coefficient. For instance, for $V=15 \mathrm{~ms}^{-1}$, we have $\bar{\mu}_{\text {norm }}=0.522, A_{\text {norm }}=0.644, B_{\text {norm }}=0.704$, $C_{\text {norm }}=1.151$. Thus, the drop of $\bar{\mu}$ by factor 0.522 , when $V$ increases from 1 to $15 \mathrm{~ms}^{-1}$, is due to: (i) the thermal softening of the flow stress in the sticking zone ( factor 0.644), (ii) the decay of $B$ (factor 0.704 ) or equivalently the magnification of the mean normal stress on the rake face $\left(F_{n}(\right.$ rake $\left.) / l_{c}\right)$ (factor $\left.1 / 0.704\right)$, and (iii) the growth of $C$ (factor 1.151). Note that the increase of $C$ is a consequence of the rising of the sticking ratio with $V$. The normal force $F_{n}$ (rake) and the contact length $l_{c}$ are both decreasing functions of $V$, however $l_{c}$ decays in a larger proportion, see Table 4; consequently, the mean normal stress $\left(F_{n}(\right.$ rake $\left.) / l_{c}\right)$ is an increasing function of $V$. The main information is that flow stress softening (decreasing of $\bar{\tau}_{S Z}$ ) and stress concentration (effect of $\left(F_{n}\left(\right.\right.$ rake) $\left.\left./ l_{c}\right)\right)$ contribute jointly and in similar proportion to the weakening of $\bar{\mu}$. The effect of the sticking ratio (embedded in $C$ ) has a lower impact and provides an opposite trend (increasing of $\bar{\mu}$ with $V$ ).

Finally, the effect of the stress exponent is analyzed in Fig. $7 \mathrm{~d}$ where three values of $\xi$ are considered ( $\xi=0.23$, flat stress profile; $\xi=0.4$; and $\xi=1$, linear profile). It appears that, for cutting speeds larger than $5 \mathrm{~ms}^{-1}$, the value of $\bar{\mu}$ is weakly affected by the stress exponent. The softening of $\bar{\mu}$ is well reproduced for all values of $\xi$ considered here.

$\bar{\mu}$ can be represented in terms of the analytical laws (15) (17) and (18) when sticking is significant, i.e. for large enough values of the sliding friction coefficient $\mu$. This was demonstrated in Fig. 7 for the case of $\mu=0.8$. This is also illustrated for $\mu=1$ in Fig. 8 where numerical estimates of $\bar{\mu}$ are compared to values obtained from Eq. (15) (with $\omega=0.9$ and $\xi=0.23$ ). It is emphasized that Eq. (15) with $\omega$ close to unity, should only be employed when contact is dominated by sticking.

The effect of the Taylor-Quinney coefficient $\beta$ on the mean friction coefficient $\bar{\mu}$ is illustrated in Fig. 6. Relationship (15) allows

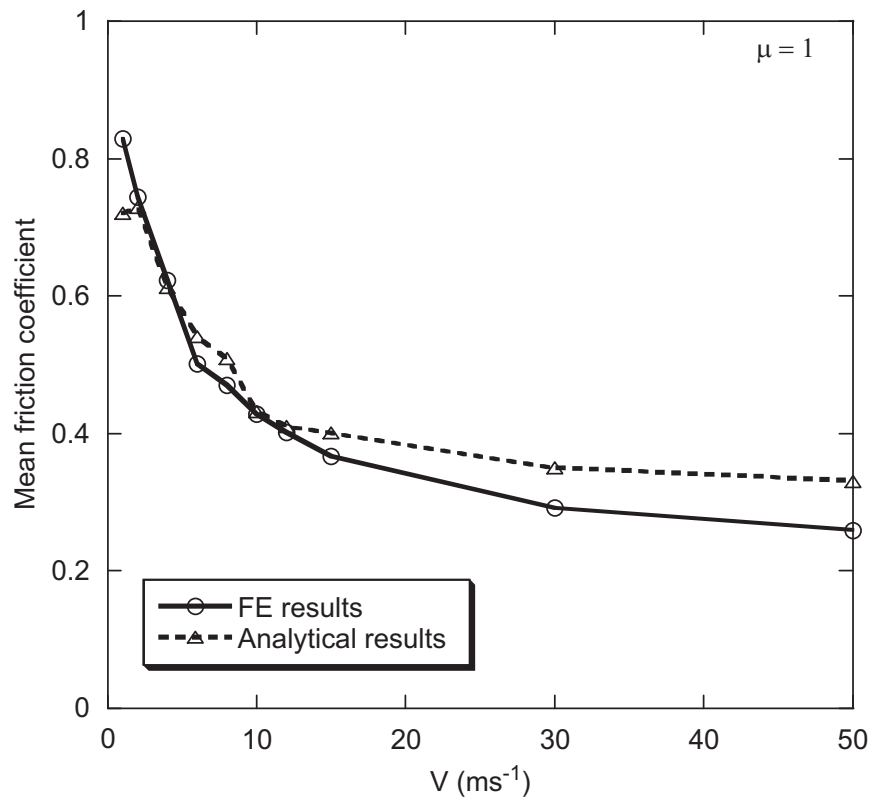

Fig. 8. Evolution of $\bar{\mu}$ with $V$ for $\mu=1$.The predictions of Eq. (15) with $\xi=0.23$ and $\omega=0.9$ (same values as in Fig. 7b) are compared to Finite Element results.
Table 7

Effect of $\beta$ on the mean friction coefficient $\bar{\mu}$. Analysis of the factors contributing to the variation of $\bar{\mu}$. Cutting conditions are those of Table 4 with $\mu=0.6$ and $V=8 \mathrm{~ms}^{-1}$.

\begin{tabular}{lllll}
\hline$\beta$ & $\begin{array}{l}A=\bar{\tau}_{S Z} \\
(\mathrm{MPa})\end{array}$ & $\begin{array}{l}1 / B=F_{n}(\text { rake }) / l_{c} \\
(\mathrm{MPa})\end{array}$ & $A B$ & $\bar{\mu}$ \\
\hline 0.9 & 440 & 859 & 0.512 & 0.446 \\
0.6 & 513 & 828 & 0.620 & 0.559 \\
\hline
\end{tabular}

us to have a better insight on how the heat conversion factor $\beta$ contributes to the softening of $\bar{\mu}$. For instance consider the test \#45 of Table 4 with $\mu=0.6, V=8 \mathrm{~ms}^{-1}$ and $\beta=0.9$. The main factors contributing to the evolution of $\bar{\mu}$ with $V$ were shown to be $A=\bar{\tau}_{S Z}$, $B=\left(1 /\left(F_{n}(\right.\right.$ rake $\left.\left.) / l_{c}\right)\right)$. The variation of $\bar{\mu}$ will be analyzed when decreasing the value of the Taylor-Quinney coefficient from $\beta_{1}=0.9$ to $\beta_{2}=0.6$. The corresponding values of $A$ and $B$ and of $\bar{\mu}$ are reported in Table 7 . It appears that $(A B)_{2} /(A B)_{1}=0.620 /$ $0.512=1.21$ is close to $\bar{\mu}_{2} / \bar{\mu}_{1}=0.559 / 0.446=1.25$. This result confirms that the factor $C$ defined above has a negligible effect on the variation of $\bar{\mu}$. In addition, it is apparent from the data of Table 7 that the variation of $\bar{\mu}$ with $\beta$ is mainly due to the factor $A=\bar{\tau}_{S Z}$. This situation is different from those of Fig. 7c where the evolution of $\bar{\mu}$ with $V$ was analyzed for the fixed value $\beta=0.9$. In that case both factors $A=\bar{\tau}_{S Z}$ and $B=\left(1 /\left(F_{n}(\right.\right.$ rake $\left.\left.) / l_{c}\right)\right)$ contributed in similar proportions to the variation of $\bar{\mu}$.

\section{Apparent friction coefficient}

The dependence of $\mu_{a p}$ with respect to $\mu$ and the cutting speed is illustrated in Fig. 9a. The cutting conditions are those of Table 4. The curvature of the cutting edge provides an additional resistance to the global chip flow; thus, it follows that $\mu_{a p}>\bar{\mu}$.

It appears that Fig. 9a and Fig. 3 show similar trends for the variation of $\mu_{a p}$ and of $\bar{\mu}$ with $V$ (for a given value of $\mu$ ). Indeed, the evolution of $\mu_{a p}$ with cutting speed is governed by the same physical mechanisms as described in Section 5.1 for $\bar{\mu}$.

The effects of the thermo-mechanical coupling factor $\beta$ are illustrated in Fig. $9 \mathrm{~b}$ for $\mu=0.6$ and appear also to be similar to those observed in Fig. 6 for $\bar{\mu}$.

Interesting features can be observed in Fig. 10 when comparing $\mu_{a p}$ to $\bar{\mu}$ for large and low friction, respectively, $\mu=0.8$ and 0.2 . Cutting conditions are those of Table 4. First, let us consider large values of the sliding friction coefficient $(\mu \geq 0.4)$. At low cutting speed $\left(V=1 \mathrm{~ms}^{-1}\right)$, it is observed that $\mu_{a p} \approx \bar{\mu}$, see Fig. 10a and Table 4 . When increasing the cutting speed a gap appears between $\mu_{a p}$ and $\bar{\mu}$ which is growing with $V$. These features can be explained as follows. Stress concentration occurs at the tool tip along an arc of circle which is scaled by the tool edge radius $R$. This stress concentration affects the level of the force exerted by the workpiece on the tool and thereby contributes to the level of the apparent friction coefficient $\mu_{a p}$. The ratio $R / l_{c}$ is a key factor for the understanding of the dependence of $\mu_{a p}$ upon $\mu$ and $V$. It appears from Table 4 that the contact length $l_{c}$ (defined as $l_{c}=I J$ in Fig. $1 \mathrm{~b}$ ) is an increasing function of $\mu$ and a decreasing function of the cutting speed. Therefore $R / l_{c}$ is a decreasing function of $\mu$. The resultant of the contact forces exerted by the workpiece on the tool is obtained by summation (integration) along the tool-workpiece interface. In this integration the vicinity of the tool edge (scaled by $R$ ) can be distinguished from the tool rake face (length $l_{c}$ ). Clearly, the relative contribution of the tool edge increases with $R / l_{c}$. Thus, from geometrical reason, the relative contribution of the tool edge radius to $\mu_{a p}$ is going to be less important at large values of $\mu$ since $R / l_{c}$ is a decreasing function of $\mu$. Consequently, for $\mu \geq 0.4, \mu_{a p}$ is given with good approximation by the ratio of the tangential and 


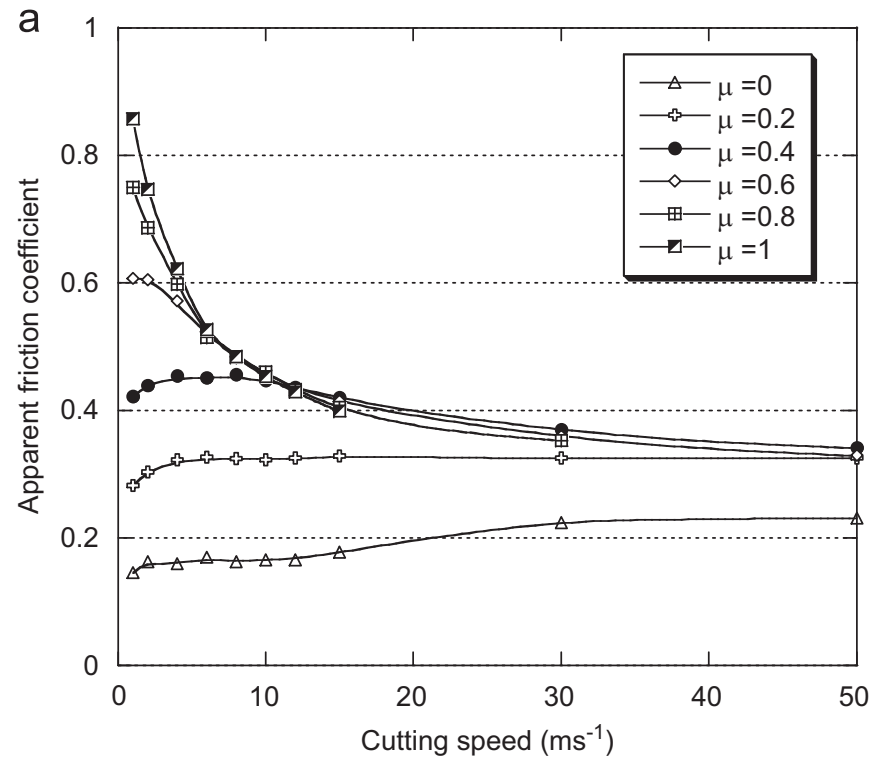

b

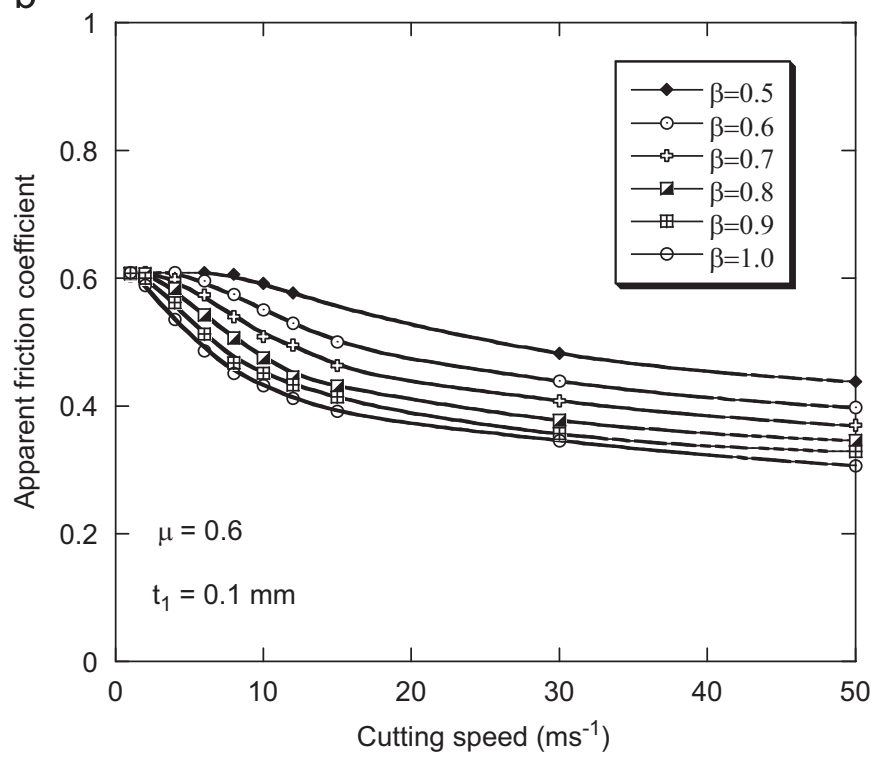

Fig. 9. (a) Effect of the cutting speed $V$ on the apparent friction coefficient $\mu_{a p}$ (ratio of the thrust and cutting forces, for $\alpha=0$ ). Cutting conditions are those of Table 4 . Various values of the sliding friction coefficient $\mu$ are considered. It can be observed that the values of $\mu_{a p}$ are merging for $\mu \geq 0.4$ and large cutting speeds (contact dominated by sticking). (b) Effect of the Taylor-Quinney coefficient $\beta$ for $\mu=0.6$ and the cutting conditions of Table 4 .

normal contact forces acting on the tool rake face (tool tip excluded) and it follows that $\mu_{a p} \approx \bar{\mu}$, at least for low cutting speeds. Why $\mu_{a p}$ and $\bar{\mu}$ happen to have distinct values at large cutting speeds as shown by Fig. $10 a$ ? As a matter of fact, for large values of $\mu$, the contact length $l_{c}$ decays rapidly with the cutting speed $V$, see Table 4 . Thus $R / l_{c}$ is larger at high velocities and the relative contribution of the tool edge radius is more significant. This implies that the gap between $\mu_{a p}$ and $\bar{\mu}$ is growing with $V$.

It is worth noting that for low values of the sliding friction coefficient $\mu$, the overall friction characteristics $\mu_{a p}$ and $\bar{\mu}$ are always significantly different, whatever is the value of the cutting speed, see Fig. 10b.

The effect of the tool edge radius $R$ on $\mu_{a p}$ and $\bar{\mu}$ is depicted in Fig. 11a for $\mu=0.8$ and $t_{1}=0.1 \mathrm{~mm}$ (other cutting conditions are those of Table 4). Two values of $R$ are considered: 15 and $30 \mu \mathrm{m}$. As
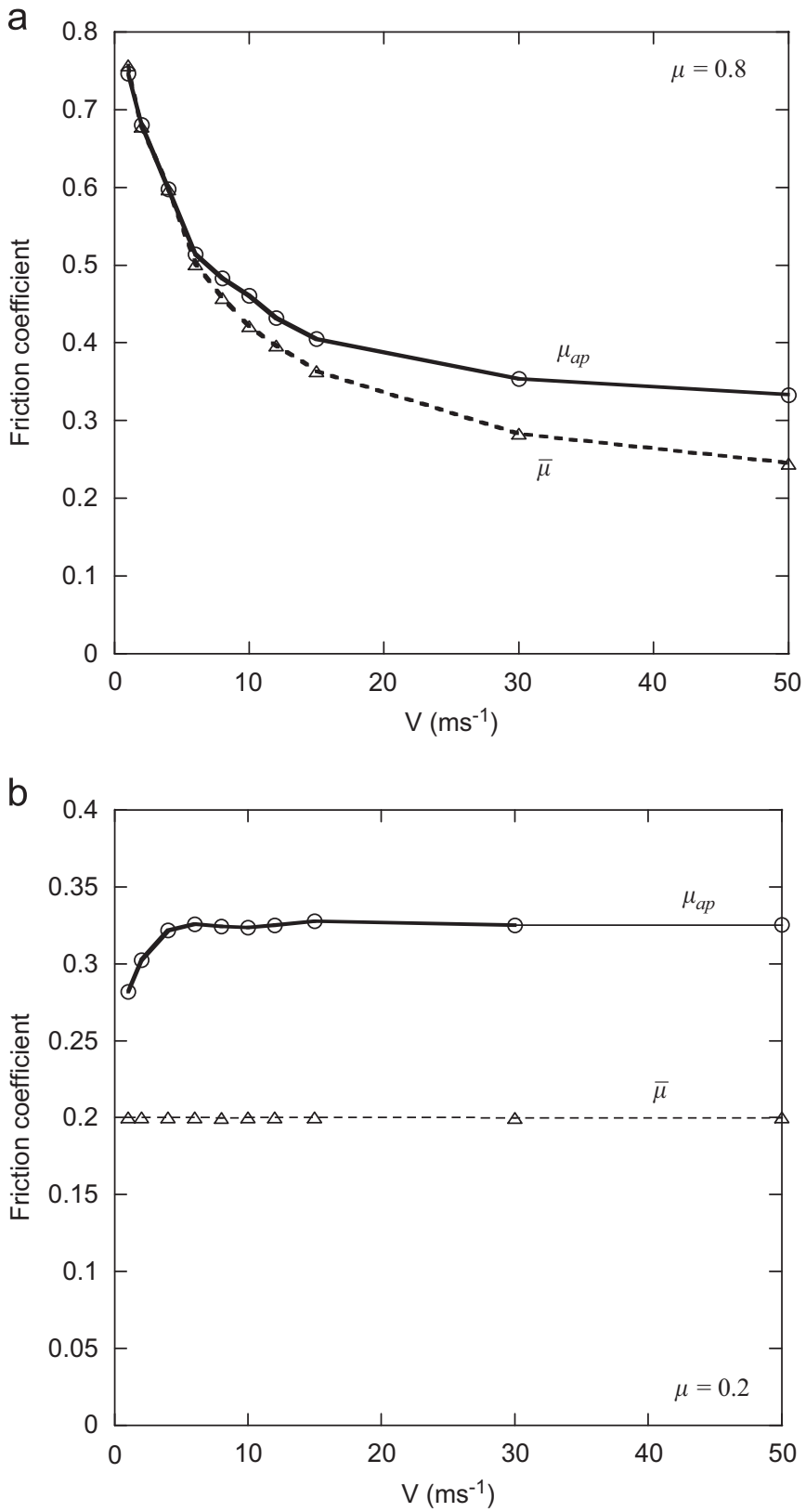

Fig. 10. Evolutions of $\mu_{a p}$ and $\bar{\mu}$ with respect to $V$ for (a) large friction $\mu=0.8$ and (b) low friction $\mu=0.2$. In general $\mu_{a p}>\bar{\mu}$ due to the effect of the tool edge radius $R$. The effect of $R$ is more important at low values of the sliding friction coefficient $\mu$ and for large cutting speeds.

expected, $\mu_{a p}$ and $\bar{\mu}$ are increasing with $R$. It appears that $\bar{\mu}$ is less affected by the tool edge radius than $\mu_{a p}$. This feature could be anticipated, as $\bar{\mu}$ solely accounts for the contact forces exerted of the flat rake face, which are weakly affected by $R$ far enough from the tool tip. The gap $\mu_{a p}-\bar{\mu}$ increases with $R$ as illustrated in Fig. 11a. For $R=0$, the values of $\mu_{a p}$ and $\bar{\mu}$ should be close, the difference being solely due to the contact forces exerted at the clearance contact. This difference is getting smaller for large clearance angles.

The dependence of $\mu_{a p}-\bar{\mu}$ upon $R / l_{c}$ is further illustrated in Fig. $11 \mathrm{~b}$ for $\mu=0.8$ and $R=15 \mu \mathrm{m}$. It appears that the variations of $\mu_{a p}-\bar{\mu}$ and $R / l_{c}$ (versus cutting speed) are following the same trends. The variation of $\mu_{a p}-\bar{\mu}$ is reported in Fig. 11c in terms of $R / l_{c}$ for $t_{1}=0.1 \mathrm{~mm}, R=15 \mu \mathrm{m}$ and for various values of $\mu$. For a given $\mu$, the cutting speed is varied according to the data reported in Table 4 and the corresponding results are represented by the same symbol 

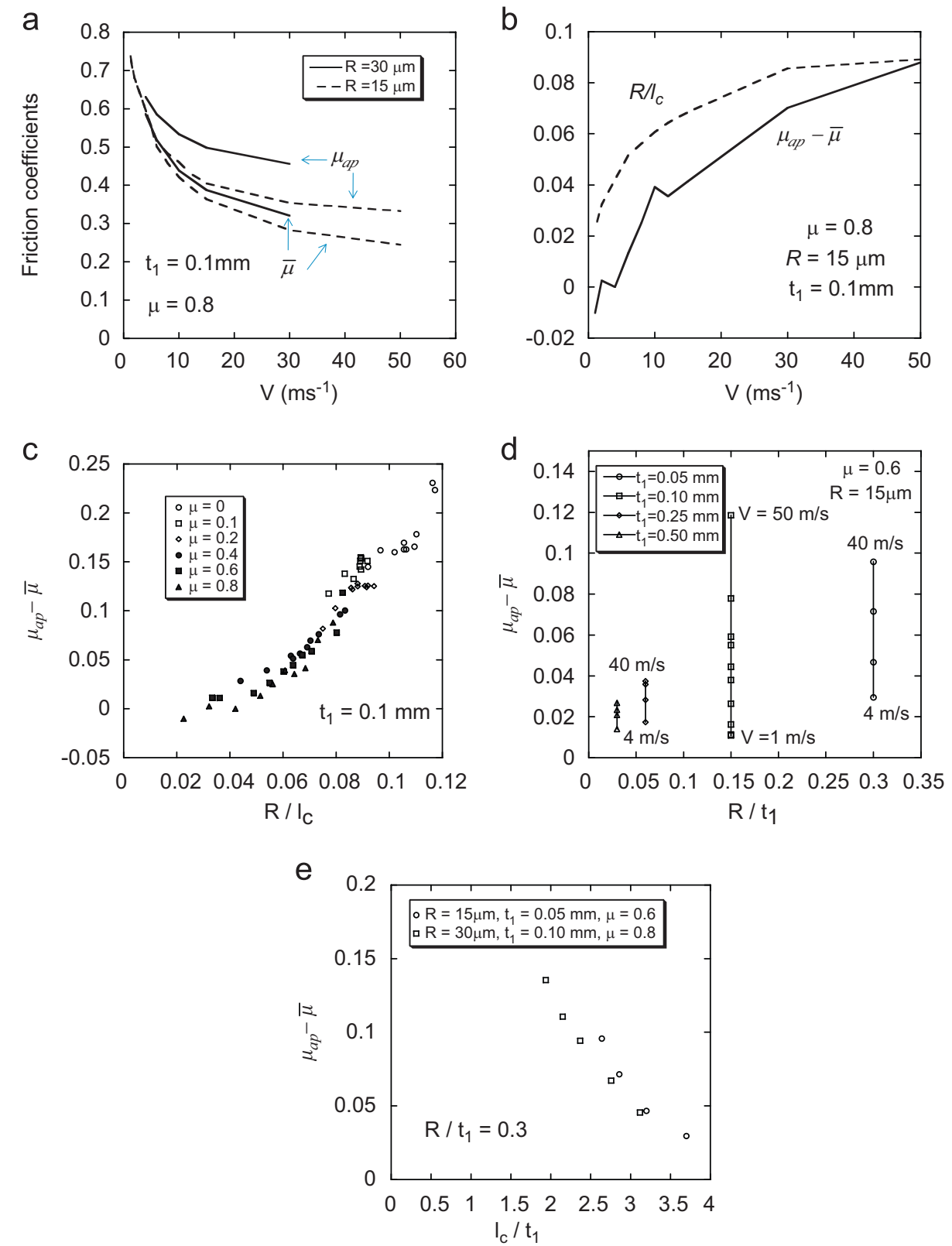

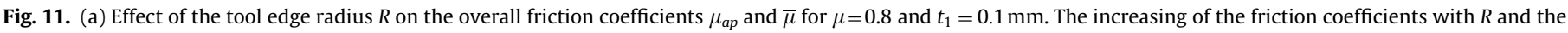

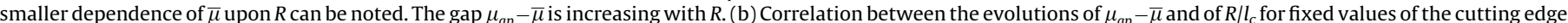

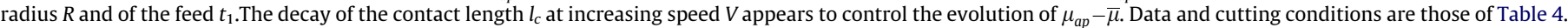

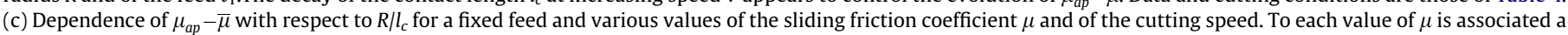

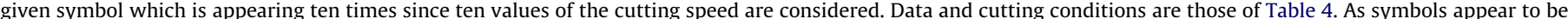

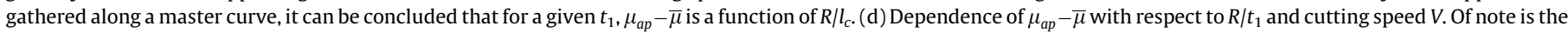

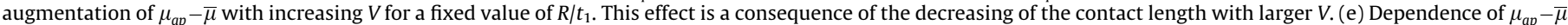
with respect to $l_{c} / t_{1}$ for two sets of cutting conditions with same value of $R / t_{1}$. These results support the fact that $\mu_{a p}-\bar{\mu}$ can be viewed as a function of $R / t_{1}$ and $l_{c} / t_{1}$.

(e.g. empty squares for $\mu=0.1$ ). The general trend revealed by Fig. $11 \mathrm{c}$ is that the gap $\mu_{a p}-\bar{\mu}$ is an increasing function of $R / l_{c}$. As $R$ is fixed at $15 \mu \mathrm{m}$, the augmentation of $\mu_{a p}-\bar{\mu}$ for a given value of $\mu$ is solely due to the decreasing of the contact length with higher cutting speeds.

The gap $\mu_{a p}-\bar{\mu}$ is not only function of $\mu$ and of the cutting speed (through the value of the contact length $l_{c}$ ), but is also depending on the uncut chip thickness $t_{1}$. This dependence is analyzed in Fig. 11d where the values of $\mu_{a p}-\bar{\mu}$ are displayed in terms of $R / t_{1}\left(t_{1}=0.05\right.$, $0.10,0.25,0.50 \mathrm{~mm}$ ) for $\mu=0.6$ and $R=15 \mu \mathrm{m}$. For a fixed value of $t_{1}$, the cutting speed is varied in the range indicated in Fig. 11d, and the corresponding values of $\mu_{a p}-\bar{\mu}$ are represented by the same symbol (e.g. a square for $t_{1}=0.10 \mathrm{~mm}$ ). As expected, it appears that $\mu_{a p}-\bar{\mu}$ is increasing with $R / t_{1}$. As discussed before, one should have $\mu_{\text {ap }} \approx \bar{\mu}$ for $R=0$. It is also observed that the effect of the cutting speed is more effective for large values of $R / t_{1}$.

Overall, $\mu_{a p}-\bar{\mu}$ can be viewed as being dependent of the geometrical cutting conditions $R$ and $t_{1}$ through the non-dimensional factors $R / l_{c}$ and $R / t_{1}$ or equivalently in terms of $l_{c} / t_{1}$ and $R / t_{1}$. This dependence is illustrated in Fig. 11e where $\mu_{a p}-\bar{\mu}$ is reported in terms of $l_{c} / t_{1}$ for $\mu=0.6$ and 0.8 and various cutting speeds. Different values of $R$ and $t_{1}$ are considered but with same ratio $R / t_{1}=0.3$. It is verified that for $R / t_{1}$ fixed, $\mu_{a p}-\bar{\mu}$ can be considered as a function of $l_{c} / t_{1}$. 
The theoretical prediction of the apparent friction coefficient $\mu_{a p}$ was compared against experimental measurements in Fig. 2c for the medium carbon steel $42 \mathrm{Cr}$ Mo4. The effect of the cutting edge radius is also analyzed in Fig. 2c.

Fig. 12 represents the evolution of $\mu_{a p}$ with the cutting speed $V$ obtained by Sutter and Molinari [19] in orthogonal cutting experiments for the same reference material ( $42 \mathrm{Cr} \mathrm{Mo} 4$ steel). Tests in the range of cutting speeds going from 0.5 to $20 \mathrm{~ms}^{-1}$ were made on an NC lathe. This lathe was equipped with a dynamometer KISTLER 9265B which permitted to measure the two components of the cutting force. A circular piece with a large diameter $\sim 125 \mathrm{~mm}$ was machined in order to obtain cutting conditions close to orthogonal machining. The range of high cutting speeds (from 10 to $90 \mathrm{~ms}^{-1}$ ) was explored by using the ballistic set-up initially developed by Sutter et al. [27] and modified in order to permit the measurement of the thrust component of the cutting force together with the longitudinal component. The same carbide tools (type SCMT 1204 08-UR 235) were used on the lathe and the ballistic set-up.

Experimental results are compared in Fig. 12 to numerical estimations of $\mu_{a p}$ and of $\bar{\mu}$ for $\mu=0.6$ and $R=0.015 \mathrm{~mm}$. The feeds in the numerical calculations are $t_{1}=0.25$ and $0.5 \mathrm{~mm}$. In the experiments, the values of the feed were $t_{1}=0.2$ and $0.5 \mathrm{~mm}$. The theoretical results are well correlated to the softening of $\mu_{a p}$ and $\bar{\mu}$ with $V$ observed in the experiments. The additional softening due to the increasing of the feed is also well reproduced by the simulations. It should be mentioned that the overall friction coefficient measured by Sutter and Molinari [19] is not exactly $\mu_{a p}$ since edge forces (obtained by linear extrapolation of the cutting and thrust forces to zero feed) were subtracted by these authors from the cutting and thrust forces. The overall friction coefficient characterized by Sutter and Molinari [19] does not correspond exactly to $\bar{\mu}$ since the net results of this operation are not strictly identical to the forces $F_{n}($ rake $)$ and $F_{t}$ (rake) applied to the flat rake face of the tool. Its value is rather in between $\mu_{a p}$ and $\bar{\mu}$, and closer to $\bar{\mu}$ than to $\mu_{a p}$.

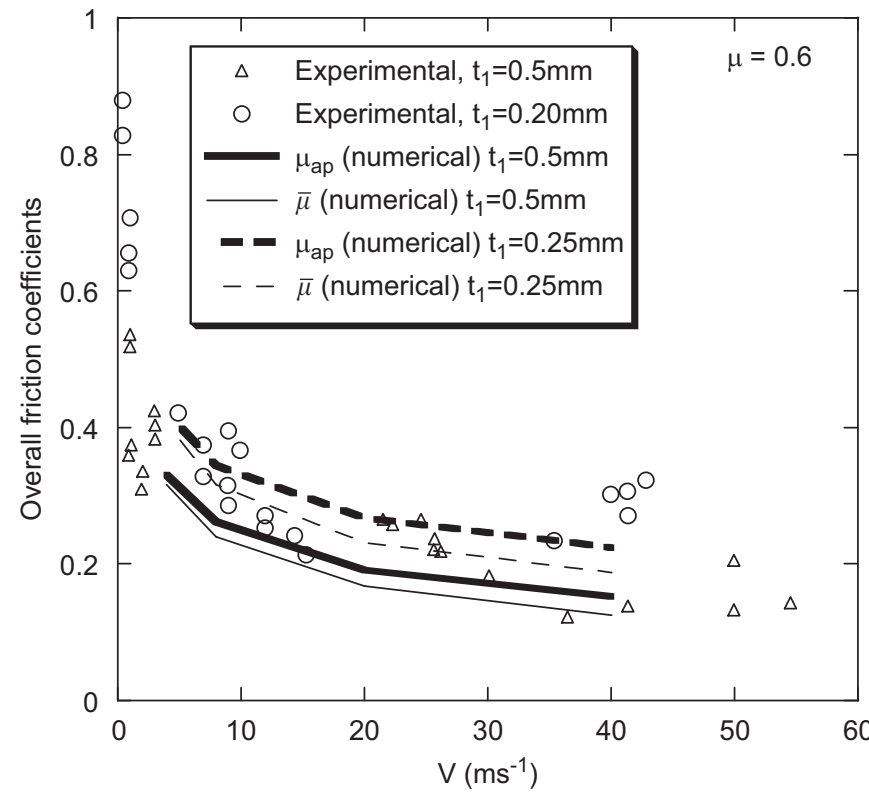

Fig. 12. Comparison of theoretical and experimental results for overall friction coefficients. The theoretical evolution of the apparent friction coefficient $\mu_{a p}$ versus cutting speed $V$ is displayed for two values of the uncut chip-thickness $t_{1}=0.25 \mathrm{~mm}$ (bold dashed curve) and $t_{1}=0.50 \mathrm{~mm}$ (bold solid curve). Numerical results relative to the mean friction coefficient $\bar{\mu}$ are represented by thin lines. The value of the sliding friction coefficient is taken to be $\mu=0.6$ and the tool edge radius is $R=0.015 \mathrm{~mm}$. The decay of $\mu_{a p}$ when increasing $t_{1}$ ( $V$ being fixed) results from the higher heating at the tool-chip interface, see Fig. 13. Experimental data obtained by Sutter and Molinari [19] with $42 \mathrm{CrMo} 4$ steel for $t_{1}=0.20$ and $0.50 \mathrm{~mm}$ are also reported. The experimental trends are well reproduced by the modeling.

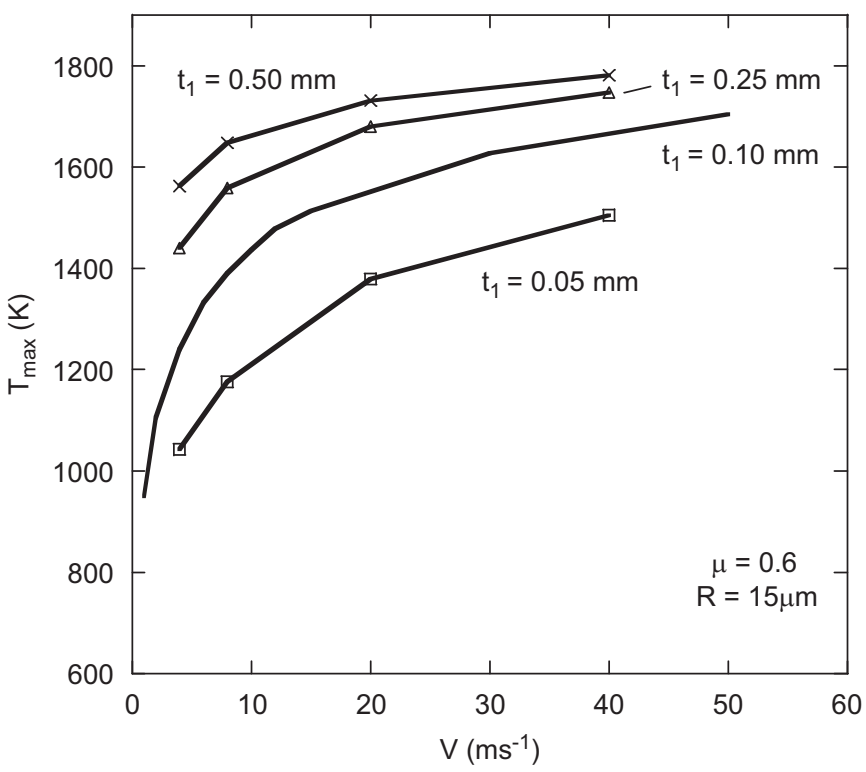

Fig. 13. Evolution of the maximum temperature $T_{\max }$ of the chip at the rake face, versus cutting speed $V$ and feed $t_{1}$. For a fixed value of $V$, the temperature increases significantly with $t_{1}$. The weakening of global friction characteristics observed for larger feeds in Fig. 12 is a consequence of the increasing of temperature with $t_{1}$ and of the resulting thermal softening of the work-material.

However, from the numerical results reported in Fig. 12, the difference between $\mu_{a p}$ and $\bar{\mu}$ is not important for the high value of the sliding friction coefficient $\mu=0.6$ considered here.

It is worth reminding that the comparison with experiments was performed in Fig. 2 with $\mu=0.8$. Nevertheless, the values of $\mu_{a p}$ corresponding to $\mu=0.6$ and 0.8 are shown in Fig. 9 to be nearly identical when the cutting speed is large enough $\left(V>10 \mathrm{~ms}^{-1}\right)$. The same observations can be made for $\bar{\mu}$, see Fig. 3 .

Alterations of overall friction characteristics generated by increasing the cutting speed and the feed are a consequence of the augmentation of the interface temperature with $V$ and $t_{1}$. The correlation with the temperature is illustrated in Fig. 13, which shows the evolution of the maximum chip temperature $T_{\max }$ (at the tool rake face) in terms of the cutting speed for various values of the feed $t_{1}$. It appears that $T_{\max }$ is an increasing function of $V$ and $t_{1}$.

\section{Temperature dependence of global friction characteristics}

As discussed in Sections 5 and 6, the evolutions of the global friction characteristics $\bar{\mu}$ and $\mu_{a p}$ are mostly controlled by thermal softening of the work-material along the tool rake face. The local sliding friction coefficient $\mu$ is assumed constant and therefore does not play any role in the variation of $\bar{\mu}$ and $\mu_{a p}$. Thus, it seems natural to consider that the dependence of $\bar{\mu}$ and $\mu_{a p}$ upon cutting conditions, is ruled at first approximation by the magnitude of the chip temperature which governs material softening at the tool interface. This temperature level will be characterized here by $T_{\max }$, the maximum chip temperature introduced previously.

Fig. 14a, describes the variation of $\mu_{a p}$ in terms of $T_{\max }$ for various cutting speeds and feeds. The cutting conditions are those of Table 4 except for the feed which is varied. The sliding friction coefficient is $\mu=0.6$. Remarkably, the results are displayed along a single master curve. A similar observation in made for $\bar{\mu}$ in Fig. 14b. These observations support the idea that, for a given work material and a given tool, the overall friction characteristics can be described by phenomenological laws of the form $\bar{\mu}\left(T_{\max }\right)$ and $\mu_{a p}\left(T_{\max }\right)$. The important result obtained here, is that the dependence of $\bar{\mu}$ and $\mu_{a p}$ 
a

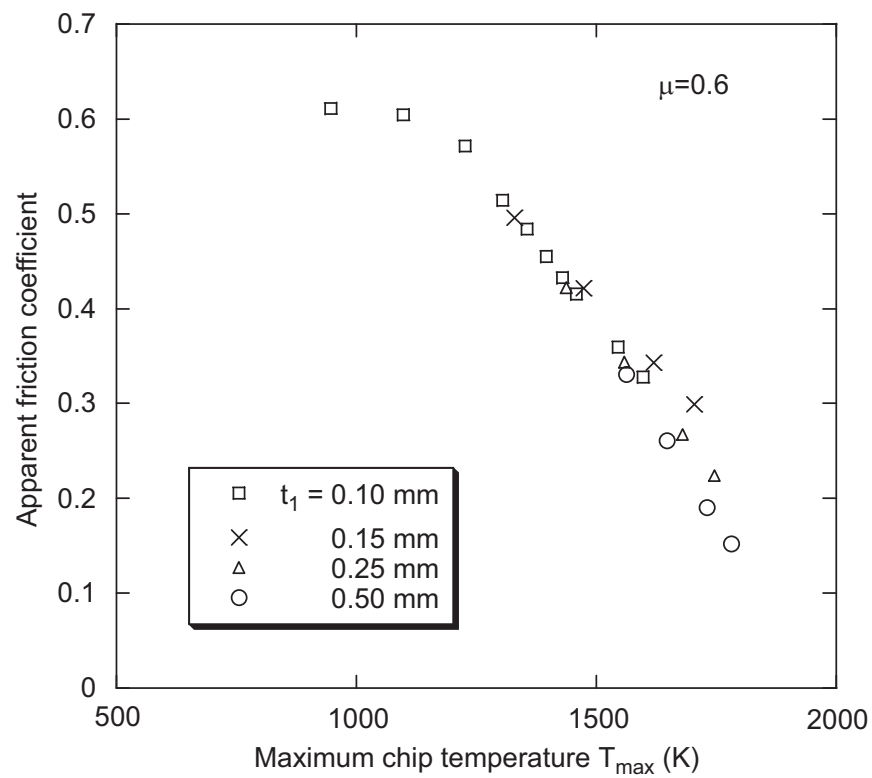

b

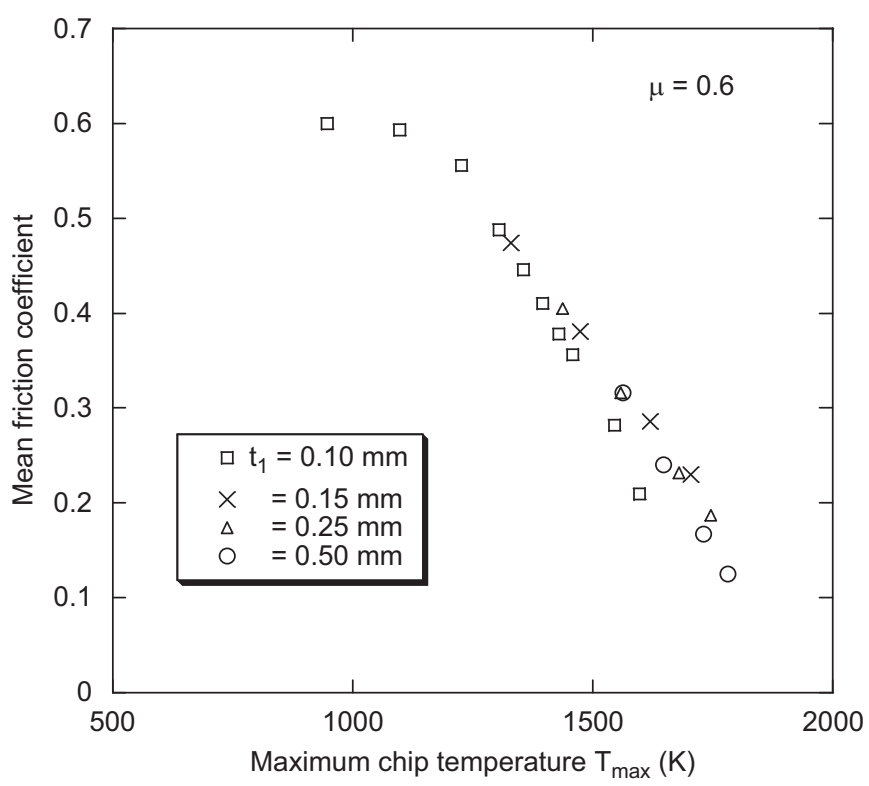

Fig. 14. Overall friction characteristics versus $T_{\max }$ (maximum chip temperature at the rake face) for various feeds $t_{1}$ and cutting speeds: (a) apparent friction $\mu_{a p}$ and (b) mean friction $\bar{\mu}$. The value of the sliding friction coefficient is $\mu=0.6$. Each symbol is associated to a given feed (e.g. squares correspond to $t_{1}=0.10 \mathrm{~mm}$ ). For $t_{1}=0.10$ $\mathrm{mm}$ the cutting speeds are $V=1,2,4,6,8,10,12,15,30,50 \mathrm{~ms}^{-1}$ (see Table 4). For other feeds we have $V=4,8,20,40 \mathrm{~ms}^{-1}$. The results for $\mu_{a p}$ happen to be gathered together along a single line which can be viewed as the master curve governing the overall constitutive response of the tool-chip interface. Consequently, the interface response can be represented in terms of the single variable $T_{\max }$ accounting for the effects of the cutting variables $V$ and $t_{1}$. Similarly, the rake face response is represented by $\bar{\mu}$ and appears to be well described by a phenomenological law depending solely upon $T_{\max }$.

with respect to cutting conditions is carried by the single parameter $T_{\max }$ characterizing the heating of the chip along the tool rake face.

The laws $\bar{\mu}\left(T_{\max }\right)$ and $\mu_{a p}\left(T_{\max }\right)$ are implicitly function of the thermo-mechanical properties of the work material and of the tool characteristics. For instance, $\bar{\mu}\left(T_{\max }\right)$ and $\mu_{a p}\left(T_{\max }\right)$ are functions of the sliding friction coefficient $\mu$. As a matter of fact, $\mu$ is expected to be smaller for a CBN tool than for uncoated carbide tool and this feature should be reflected in the laws governing the evolution of overall friction coefficients. The effect of $\mu$ on $\bar{\mu}\left(T_{\max }\right)$ is illustrated
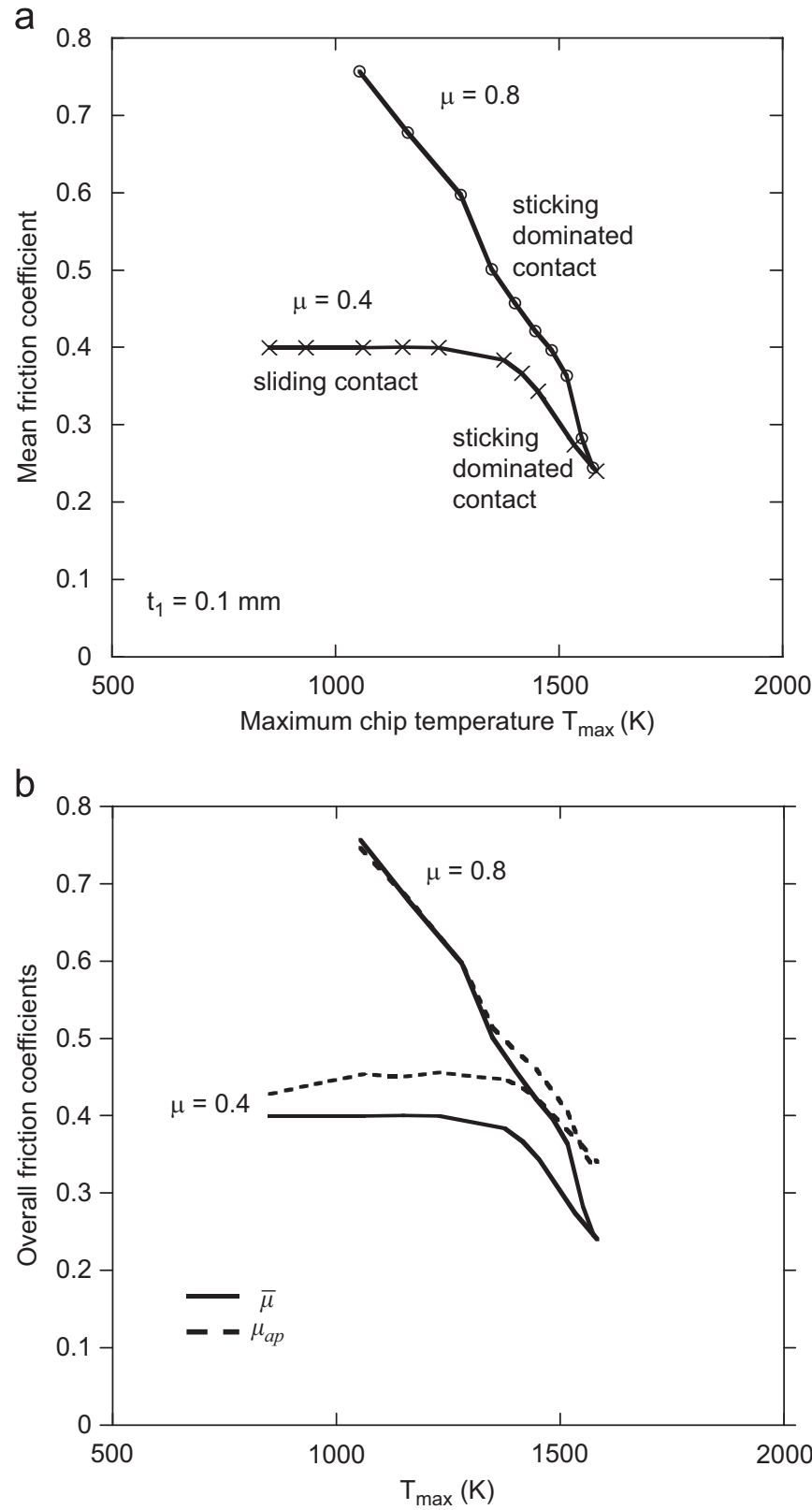

Fig. 15. (a) Mean friction coefficient $\bar{\mu}$ in terms of the maximum chip temperature $T_{\max }$ for two values of the sliding friction coefficient $\mu=0.4$ and 0.8 . Cutting conditions are defined by $t_{1}=0.10 \mathrm{~mm}, R=0.015 \mathrm{~mm}$ and the cutting speeds $V=1,2,4,6,8,10,12,15,30,50 \mathrm{~ms}^{-1}$. To each cutting speed is associated a value of $T_{\max }$ (crosses for $\mu=0.4$, circles for $\mu=0.8$ ). For $\mu=0.4$ two distinct contact regimes can be distinguished. At low cutting speeds (small values of $T_{\max }$ ), the contact is governed by sliding friction and we have $\bar{\mu}=\mu$. At higher speeds, a transition is observed towards a contact regime dominated by sticking with a drop of $\bar{\mu}$ controlled by thermal softening of the work-material. For $\mu=0.8$, the sliding regime is not apparent for the range of cutting speeds explored here. It is worth noting the merging of the values of $\bar{\mu}$ in the sticking dominated regime.(b) Comparison of $\bar{\mu}$ and $\mu_{a p}$. The same observations made in (a) for $\bar{\mu}$ holds for $\mu_{a p}$.

in Fig. 15a. For $\mu=0.4$, two distinct regimes are observed. For temperatures smaller than $1370 \mathrm{~K}$ the mean friction coefficient $\bar{\mu}$ is independent of $T_{\max }$. The plateau in the curve associated to $\mu=0.4$, corresponds to a pure sliding regime (no sticking) for which necessarily $\bar{\mu}=\mu=0.4$. For sticking to occur, the temperature has to become large enough in order to induce a sufficient drop of the flow stress of the work material. The decreasing branch of the curve $\bar{\mu}\left(T_{\max }\right)$ observed for $\mu=0.4$ in Fig. $15 \mathrm{a}$ is related to the occurrence of sticking contact and describes the softening of $\bar{\mu}$ due 
to thermal effects. For the larger friction $\mu=0.8$, the descending branch due thermal softening is solely observed. This does not mean that the plateau regime (associated to pure sliding) does not exist. To make the plateau regime $(\bar{\mu}=\mu)$ apparent, cutting speeds lower than $1 \mathrm{~ms}^{-1}$ should be explored. It should be noted that the descending branches associated to $\mu=0.4$ and 0.8 merge at large temperatures (i.e. high cutting speeds). This point was discussed before. The effect of $\mu$ appears to be wiped out when contact is dominated by sticking. This happens when the heating of the chip at the tool interface is large enough, i.e. at high cutting speeds. Then, $\bar{\mu}$ and $\mu_{a p}$ are nearly independent of $\mu$, as apparent in Figs. 3 and 9 for $\mu \geq 0.4$. The specific effect of the tool disappears in favor to the sole contribution of the thermo-mechanical properties of the work-material. In other words, working with a CBN tool or a carbide tool has no specific effects on the values of the overall frictions coefficients if the interface temperature is sufficiently high. It must be reminded that these results have been obtained under the assumption that the sliding friction coefficient $\mu$ is not affected by the cutting conditions.

Results for the apparent friction coefficient $\mu_{a p}$ (dashed lines) are compared to those of $\bar{\mu}$ (solid lines) in Fig. 15b. Similarities between the variations of $\mu_{a p}$ and $\bar{\mu}$ appear clearly. As discussed previously, the gap $\mu_{a p}-\bar{\mu}$ results from the effect of the tool edge radius. For a fixed value of $\mu$, the gap $\mu_{a p}-\bar{\mu}$ increases with the cutting speed (i.e. with $T_{\max }$ ), in agreement with Fig. 11a, and is a decreasing function of $\mu$, as in Fig. 11c.

From the above results, it is appealing to describe the dependence of $\mu_{a p}$ and $\bar{\mu}$ with respect to the cutting conditions in terms of phenomenological constitutive laws. It is proposed to characterize the functional relationship between the mean friction coefficient and $T_{\max }$ as

$\bar{\mu}=S_{1}\left(T_{1}^{*}-T_{\max }\right)\left\{1-\exp \left[-\left(\frac{\mu}{S_{1}\left(T_{1}^{*}-T_{\max }\right)}\right)^{n_{1}}\right]\right\}^{1 / n_{1}}$ for $T_{\max } \leq T_{1}^{*}$

$\bar{\mu}=0$ for $T_{\max } \geq T_{1}^{*}$

For small values of $T_{\max }$, it follows from Eq. (19) that $\bar{\mu} \approx \mu$. When $T_{\text {max }}$ increases towards $T_{1}^{*}$ it occurs that $\bar{\mu} \approx S_{1}\left(T_{1}^{*}-T_{\max }\right)$ and that $\bar{\mu}$ decreases linearly with $T_{\max } . T_{1}^{*}$ is a reference temperature and the factor $S_{1}>0$ controls the rate of decay of $\bar{\mu}$ at large temperatures. The third parameter introduced in law (19) is the exponent $n_{1}$ which governs the sharpness of the transition between sliding contact and the sticking dominated regime. The evolution of $\bar{\mu}$ with respect to $T_{\max }$ is displayed in Fig. 16a. Three values of the sliding friction coefficient are considered: $\mu=0.4,0.6$ and 0.8 . The numerical results are represented by symbols (for instance a square for $\mu=0.8$ ). Cutting conditions are those of Table 4 . The results associated to law (19) are given by solid lines. The values of the parameters are: $S_{1}=0.0009\left(K^{-1}\right), n_{1}=10, T_{1}^{*}=1930(K)$. In law (19) the contribution of the tool is represented by the sliding friction coefficient $\mu$, while $T_{1}, S_{1}$ are related to the thermal softening of the work material.

Thus, the consequence of replacing a tool with low friction $\mu=0.4$ by a tool with higher friction $\mu=0.8$ can be analyzed in Fig. 16a. At high temperature (high cutting speeds, large feed), there is no effect of the tool since the contact is dominated by sticking and by thermal softening of the work material. However at lower temperatures, the effect of the tool is quite visible as the contact is governed by sliding $(\bar{\mu}=\mu)$.

Similar results are shown for $\mu_{a p}$ in Fig. 16b. The phenomenological law governing the temperature dependence of $\mu_{a p}$ is taken in the form:

$\mu_{a p}=S_{2}\left(T_{2}^{*}-T_{\max }\right)\left\{1-\exp \left[-\left(\frac{\mu_{a p}^{0}}{S_{2}\left(T_{2}^{*}-T_{\max }\right)}\right)^{n_{1}}\right]\right\}^{1 / n_{1}}$ for $T_{\max } \leq T_{2}^{*}$

$\mu_{a p}=0$ for $T_{\max } \geq T_{2}^{*}$

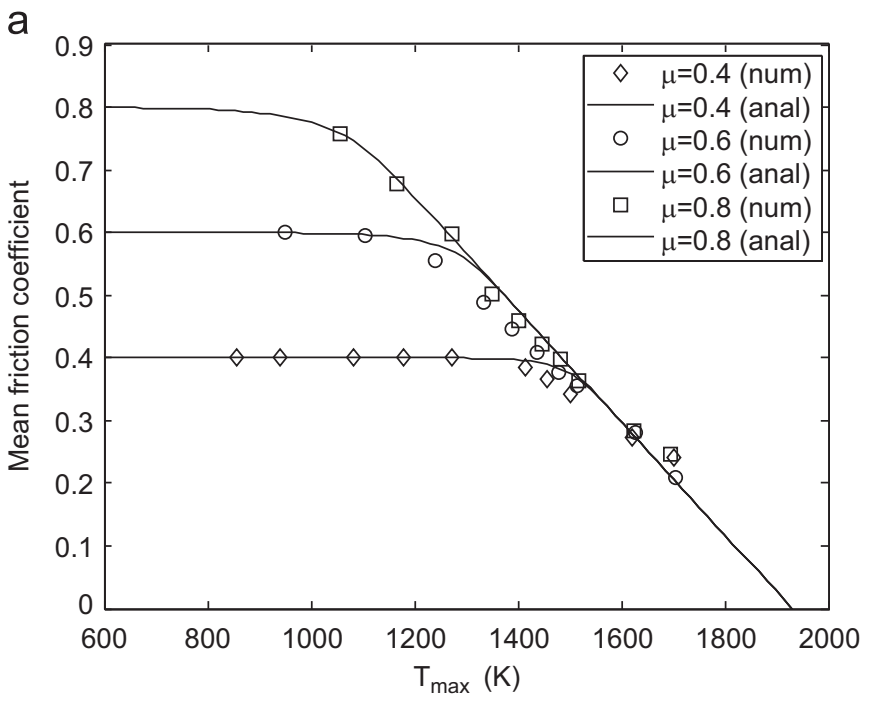

b

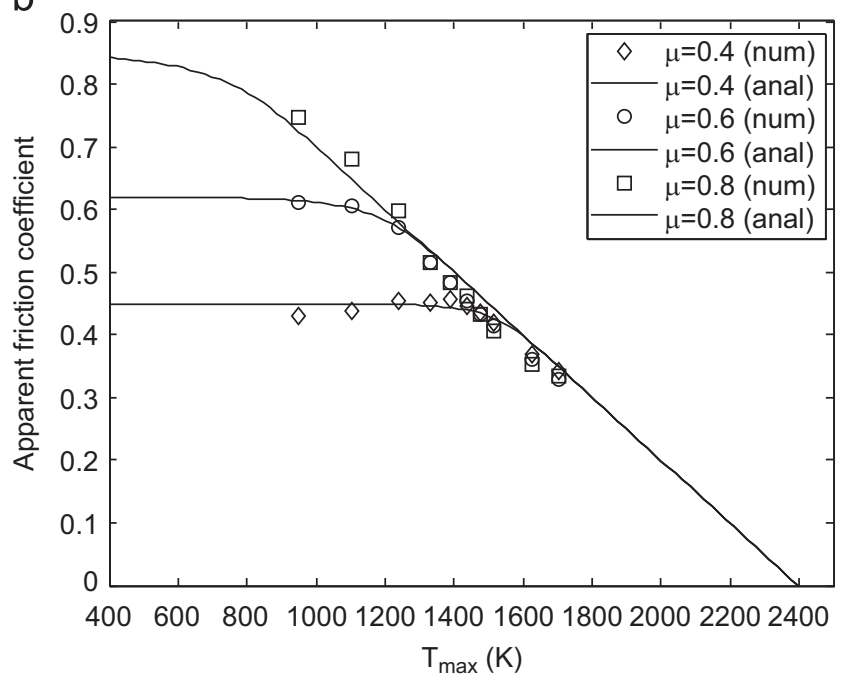

Fig. 16. (a) Mean friction coefficient $\bar{\mu}$ in terms of the maximum chip temperature $T_{\text {max }}$ : analytical results given by the phenomenological law (19) are represented against numerical results. (b) Apparent friction coefficient $\mu_{a p}$ : analytical results given by the phenomenological law (20) are represented against numerical results.

The parameters corresponding to Fig. $16 \mathrm{~b}$ are: $S_{2}=0.0005\left(K^{-1}\right)$, $n_{1}=10, T_{2}^{*}=2400(K)$. It can be noted that the same value of $n_{1}$ is used for laws (19) and (20). For small values of $T_{\max }$ relationship (20) gives $\mu_{a p}=\mu_{a p}^{0}$. The values of the parameter $\mu_{a p}^{0}$ are 0.45 for $\mu=0.4,0.62$ for $\mu=0.6$ and 0.85 for $\mu=0.8$. They are close to $\mu$. The effect of the tool edge radius is embedded into $\mu_{a p}^{0}$, this is why $\mu_{a p}^{0}>\mu$.

Analytical forms (19)-(20) proposed for $\bar{\mu}\left(T_{\max }\right)$ and $\mu_{a p}\left(T_{\max }\right)$ can be naturally improved if one has additional results at high temperatures. For instance it might occur that the decay of $\bar{\mu}\left(T_{\max }\right)$ and of $\mu_{a p}\left(T_{\max }\right)$ at large temperature is not exactly linear. In that case the term $T_{2}-T_{\max }$ in (19) and (20) could be replaced by a nonlinear function of $T_{2}-T_{\max }$, for instance a power-law of the form $\left(T_{2}-T_{\max }\right)^{q}$.

The characterization of the overall friction properties of the tool by a temperature dependent friction law was introduced by Moufki et al. [28] in their thermo-mechanical modeling of the orthogonal cutting process. They used the mean temperature $\bar{T}_{\text {int }}$ along the tool rake face to characterize the heating of the chip. In fact, taking $\bar{T}_{i n t}$ in place of $T_{\max }$ as the characteristic variable governing the evolution of the overall friction does not matter much as both variables are 
related. The results shown in this section provide a justification and an explanation for using temperature dependent friction laws in the modeling of machining. It should be noted, that the Oxley' model [8] provides a good description of the machining process when contact is mostly controlled by sticking but cannot describe the transition from sliding to sticking contact observed for instance for $\mu=0.4$ in Figs. 15 and 16 .

\section{Contact length}

The contact length is an important parameter which affects the temperature distribution at the tool rake face, the chip curling, the stress level applied on the tool and finally the wear and the mechanical resistance of the tool [29-30].

The contact length is defined as the extension of the tool-chip contact on the rake face of the tool: $l_{c}=I J$, see Fig. $1 \mathrm{~b}$. The evolution of the contact length $l_{c}$ in terms of the cutting speed is illustrated in Fig. 17a for different values of the sliding friction coefficient $\mu$. Cutting conditions and data are those of Table 4. The uncut chip thickness is $t_{1}=0.1 \mathrm{~mm}$ (see Fig. $1 \mathrm{~b}$ ), the cutting edge radius is $R=0.015 \mathrm{~mm}$ and the thermal conductance of the tool-chip interface is $\kappa=2000 \mathrm{Wm}^{-2} \mathrm{~K}^{-1}$. The contact length $l_{c}$ appears in Fig. 17a to be a decreasing function of the cutting speed and an increasing function of $\mu$. These trends are in agreement with experimental observations; see for example Gad et al. [31].

Experimental data obtained by Sutter and Ranc [32] for steels at high cutting speeds are well correlated with the numerical results displayed in Fig. 17a. For 42CrMo4 steel, the contact length was found to be $l_{c}^{\text {exp }}=0.62 \mathrm{~mm}$ at the cutting speed $V=16 \mathrm{~ms}^{-1}$, the uncut chip-thickness $t_{1}=0.3 \mathrm{~mm}$ and the rake angle $\alpha=0^{\circ}$. As the $42 \mathrm{CrMo} 4$ steel is also the reference material in our numerical simulations it is of particular interest to compare experimental and theoretical results. From the data of Table 6 , the value of the contact length is estimated as $l_{c}^{\text {theor }}=0.45 \mathrm{~mm}$ for $V=16 \mathrm{~ms}^{-1}$ and $t_{1}=0.25 \mathrm{~mm}$. The ratio $l_{c}^{\exp } / t_{1}=2.07$ obtained experimentally is in good agreement with $l_{c}^{\text {theor }} / t_{1}=1.80$.

Fig. 17a reveals the same trends as those of Fig. 9a for $\mu_{a p}$, i.e. the contact length becomes weakly dependent upon the values of $\mu$ for $\mu \geq 0.4$ and is nearly independent of $V$ at large cutting speeds. It is referred to Section 5.1 for the discussion of the physical mechanisms underlying these features.

Different analytical models or empirical approaches have been proposed to evaluate $l_{c}$. It is worth to compare these models to the results of the numerical calculations. Using the slip line theory, Lee and Shaffer [33] obtained the following result:

$l_{c} / t_{1}=\frac{\sqrt{2}}{\sin (\phi) \cos (\pi / 4+\phi-\alpha)}$
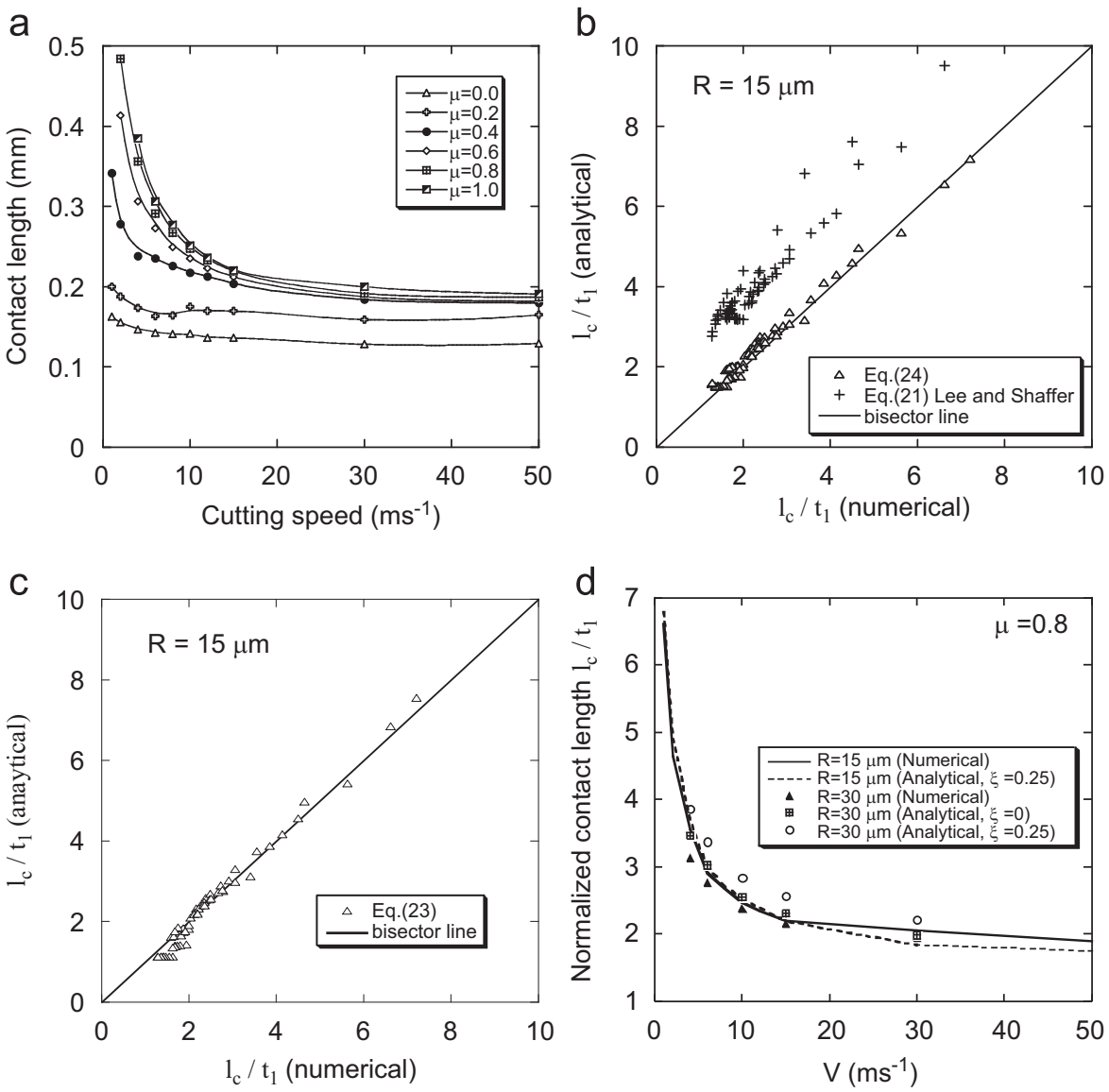

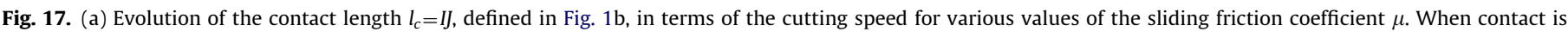

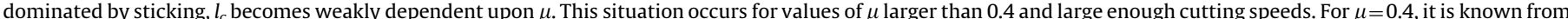

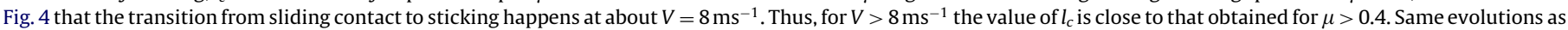

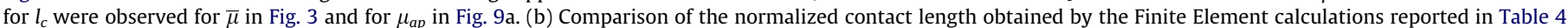

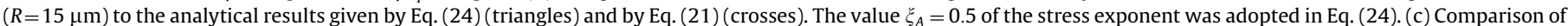

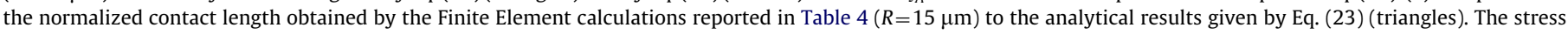

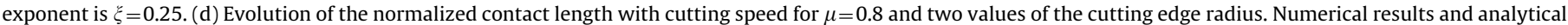
results obtained with Eq. (23) are in very good agreement. 
where the shear angle is given by $\tan (\phi)=\left(t_{1} / t_{2}\right)$. The chip thickness $t_{2}$ is defined in Fig. 1b. Empirical formulations for $l_{c}$ were given by Abuladze [34], Poletika [35], and Marinov [36].

Of note is the simple relationship proposed by Toropov and Ko [37] and Kato et al. [38]:

$l_{c} / t_{1}=2$

The contact length can be also evaluated by considering the equilibrium with respect to the tool tip of the moment of the contact forces exerted on a given chip-domain. It is useful here to define the angles $\phi_{A}$ and $\phi^{*}$ shown in Fig. 1b. The transition between the horizontal free surface of the workpiece and the chip is insured by a small curved line. Near this transition zone, the free surface of the chip can generally be represented by a straight segment. The extension of this segment intercepts at $I_{1}$ the horizontal line associated to the workpiece surface, see Fig. 1b. A more detailed characterization of $I_{1}$ is found in Fig. 24 of Appendix C. The segment $I_{1} A$ which is tangent to the tool tip at $A$ is considered. The inclination angles of $I_{1} A$ and of $I_{1} I$ with respect to horizontal are, respectively, denoted as $\phi_{A}$ and $\phi^{*}$. Intersections of $I_{1} A$ and of $I_{1} I$ with the free surface are denoted, respectively, as $B_{A}$ and $B^{*}$. The points $B_{A}$ and $B^{*}$ are different in general, but the resolution of Fig. 1b does not allow us to represent them as distinct points.

Let us consider the chip domain $D_{1}$ delimitated by the contour $J I I_{1} B_{*} C C^{\prime} J$ including the segment $I B_{*}$ with inclination angle $\phi^{*}$, see Fig. $1 \mathrm{~b}$. Another option would be to select the domain $D_{2}$ defined by the contour $J I A I_{1} B_{A} C C^{\prime} J$.

Consider first the domain $D_{1}$. The normal and tangential forces exerted on $I J$ are $F_{n}\left(\right.$ rake) and $F_{t}($ rake $)$. The mean friction coefficient associated to the contact zone $I J$ is $\bar{\mu}=F_{t}($ rake $) / F_{n}($ rake $)$. Considering that the moment at $I$ of the external forces exerted on the domain $D_{1}$ vanishes, it follows in a way similar to [28] that

$l_{c} / t_{1}=\frac{\xi+2}{2} \frac{\sin \left(\phi^{*}+\bar{\lambda}-\alpha\right)}{\sin \left(\phi^{*}\right) \cos (\bar{\lambda})}$

$\bar{\lambda}$ is the friction angle defined by $\bar{\mu}=\tan (\bar{\lambda})$. To derive this result, it has been assumed that (i) the normal stress distribution along $I J$ is given by Eq. (11), where $\xi$ is the stress exponent and (ii) the normal stress distribution along the segment $I B_{*}$ is uniform.

If the domain $D_{2}$ is selected, the resultant force exerted on the boundary $A J$ of the tool is equal to the cutting force $\underline{F}=-F_{C} \underline{e}_{Y}+F_{T} \underline{e}_{X}$, in first approximation, if the point $A$ is considered to be far enough on the right of the tool edge, see Fig. $1 \mathrm{~b}$, so that most of the tool edge is encompassed in the domain $D_{2}$. The following result is derived in Appendix D:

$\frac{l_{c}+R}{t_{1}}=\frac{\xi_{A}+2}{2} \frac{\sin \left(\phi_{A}+\lambda_{a p}-\alpha\right)}{\sin \left(\phi_{A}\right) \cos \left(\lambda_{a p}\right)}$

$\phi_{A}$ is the inclination angle of the plane $A I_{1}$ and $\lambda_{a p}=\arctan \left(\mu_{a p}\right)$ is the apparent friction angle.

In Fig. $17 \mathrm{~b}$, the predictions of $l_{c} / t_{1}$ given by Eq. (24) are displayed versus the values of $l_{c} / t_{1}$ obtained numerically, considering all the tests reported in Table 4. Values of $\phi_{A}, \mu_{a p}$ used in Eq. (24) are given in Table 4 . The same stress exponent $\xi_{A}=0.5$ was taken for all tests. Results given by law (21) of Lee and Shaffer [33] are also shown. It appears that the estimates obtained from relationship (24) are in good correlation with numerical results (triangular symbols are close to the bisector line). Relationship (21) predicts correct trends but there is a significant gap with respect to numerical results. Results of models [34-36] are not shown here as they provide results close to those obtained with law (21). It must be noted that all the models [33-36] consider that $l_{c} / t_{1}$ is sole function of the shear angle $\phi$ and of the rake angle $\alpha$. However, relationships (23) and (24) account explicitly for the effect of the overall friction characteristic $\bar{\mu}$ (or $\mu_{a p}$ ) and of the parameter $\xi$ (or $\xi_{A}$ ) related to the normal stress distribution. Taking $\phi$ in place of $\phi_{A}$ in Eq. (24) would provide results similar to those of Fig. 17b with however a slight decrease of the quality of results.

Finally the analytical results corresponding to the model (23) are represented in Fig. 17c for all the tests reported in Table 4 $(R=0.015 \mathrm{~mm})$. The stress exponent is $\xi=0.25$, a value smaller than $\xi_{A}=0.5$ used for model (24). This is in keeping with the fact that a stress-peak was observed in our simulations at the rounded edge of the tool. The value $\xi=0.25$ used in Eq. (23) refers to the stress distribution along the flat rake face, line $I J$ in Fig. $1 \mathrm{~b}$, which excludes the stress-peak existing at the tool tip. A small value of the stress exponent $\xi$ corresponds to a flat stress profile. It should be mentioned that the parameter $\xi$ enters into the results through the term $\xi+2$. Thus, the value of $l_{c}$ would be decreased by only about $12 \%$ by setting $\xi=0$ in place of $\xi=0.25$. Therefore, for relatively flat stress profiles the results are not much affected by the precise value of $\xi$.

In the range of high cutting speeds and for $\mu \geq 0.4$, it is observed in Fig. 17a that $l_{c} / t_{1} \approx 1.9$. This value is quite close to those of the model proposed by Toropov and Ko [37] and Kato et al. [38], Eq. (22).

On the practical point of view, relationship (24) is easier to use than Eq. (23) since the value of $\mu_{a p}$ is directly accessible from experiments.

The effect of the tool edge radius is quantified in Fig. 17d where the evolution of $l_{c} / t_{1}$ is reported in terms of the cutting speed for two values of $R(15$ and $30 \mu \mathrm{m})$. The effect of $R$ appears to be relatively weak when comparing numerical results. Analytical results obtained by using relationship (23) are also shown. As in Fig. $17 \mathrm{c}$, the value of the stress exponent (characterizing the stress distribution along the rake face $I J$ ) is taken to be $\xi=0.25$. However, for $R=30 \mu \mathrm{m}$ the stress distribution is expected to be flatter than for $R=15 \mu \mathrm{m}$. The stress exponent for the larger radius should be lower than $\xi=0.25$. To quantify the effect of a lower stress exponent, results associated to $\xi=0$ and $R=30 \mu \mathrm{m}$ are reported in Fig. 17d. As remarked before, for low values of the stress exponent, the precise value of $\xi$ does not change much the results.

The weak effect of $R$ on $l_{c}$ could be expected from the examination of relationship (23) and the fact that $\bar{\mu}$ is slightly sensitive to $R$, see Fig. 11a. On the contrary, $\mu_{a p}\left(\right.$ and $\left.\xi_{A}\right)$ is more dependent upon $R$, Fig. 11a. Therefore, the right hand side of Eq. (24) is affected by $R$. However, $R$ is also present in the left hand side of Eq. (24) in a way that insures the consistency of the results with those of Eq. (23).

\section{Contact forces}

Contact forces exerted onto the tool are important global characteristics that embody the resistance of the work material to plastic flow and the tribological properties of the tool-chip interface. The link between the cutting and thrust forces and contact conditions is analyzed by numerical and analytical means. A similar investigation is also carried out for the contact forces exerted on the flat part of the tool face (round edge excluded).

\subsection{Cutting and thrust forces}

The evolution of the cutting force $F_{C}$ and of the thrust (feed) force $F_{T}$ is represented in Fig. 18a and b in terms of the cutting speed $V$ for various values of the sliding friction coefficient $0 \leq \mu \leq 1$. Cutting conditions are those of Table 4 , i.e. the rake angle, the feed and the tool edge radius are, respectively, $\alpha=0, t_{1}=0.1 \mathrm{~mm}$ and $R=15 \mu \mathrm{m}$. The general trends concerning the variation of $F_{C}$ with respect to the cutting speed and $\mu$ are similar to those of $l_{c}$ shown in Fig. 17a.

As expected, for a given value of $V$ the cutting and thrust forces are increasing with the friction coefficient $\mu$. For a fixed value of $\mu$, the cutting and thrust forces are decreasing functions of $V$. The drop 

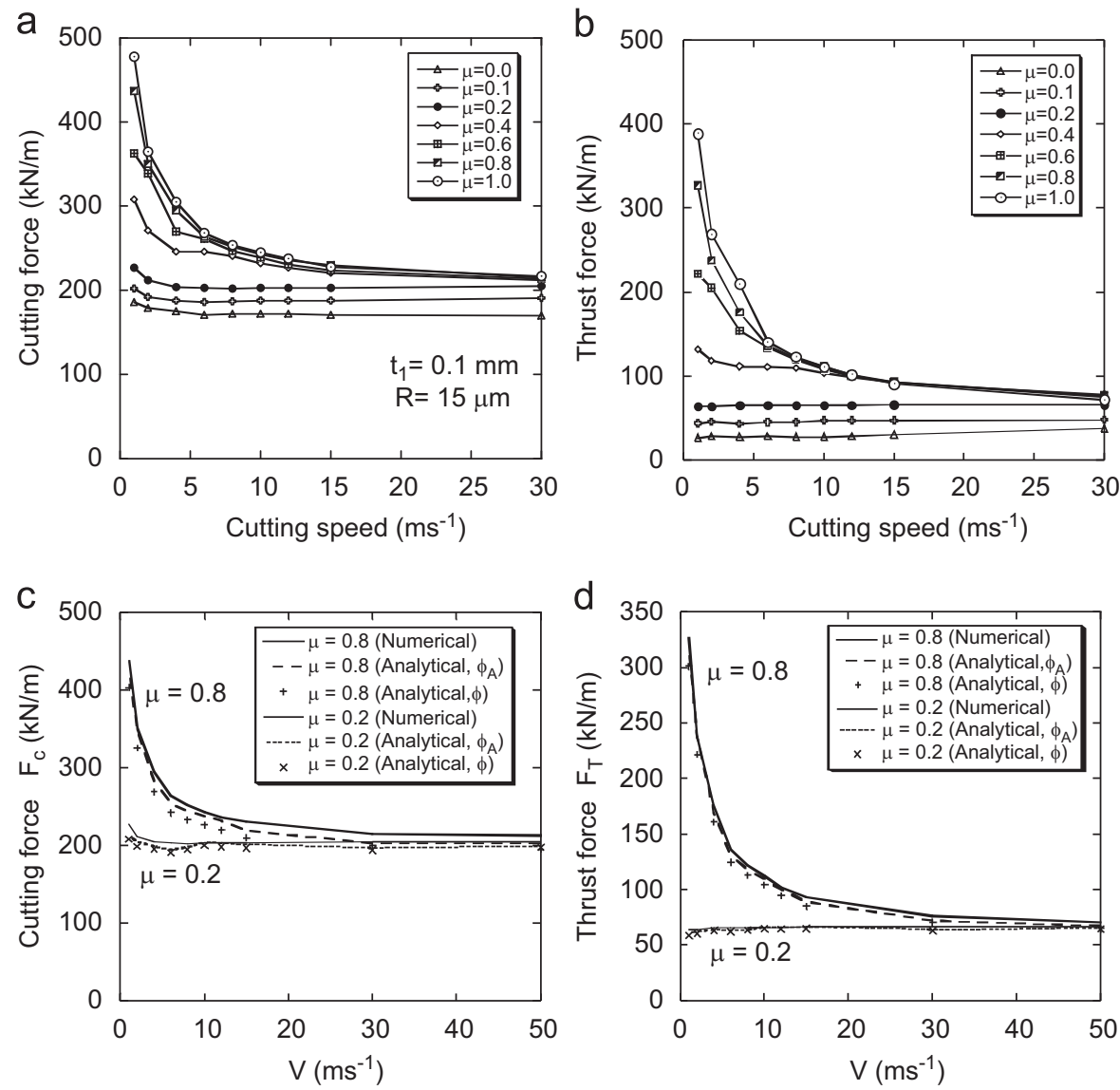

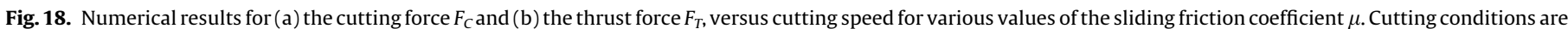

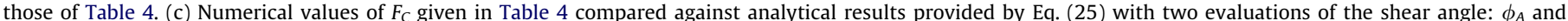

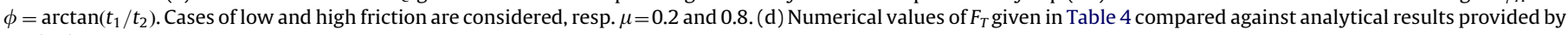
Eq. (26).

of the forces is negligible for low friction coefficient and is about $55 \%$ for $\mu=1$. For the medium carbon $42 \mathrm{CrMo} 4$ steel the theoretical predictions of $F_{C}$ and $F_{T}$ with $\mu=0.8$ were shown in Fig. 2 to be in good correlation with experimental measurements.

The level of the cutting and thrust forces is the result of the interplay between the material response within the primary shear zone (where the chip is formed) and the behavior of the tool-chip interface. The evolution of the cutting and thrust forces in terms of $V$ are similar to those of $\mu_{a p}$ for large values of the sliding friction coefficient. However it is not exactly so for $\mu \leq 0.4$, since $\mu_{a p}$ is slightly increasing for small values of the cutting speed, while the cutting force decreases.

In the analytical modeling of cutting, it is useful to refer to the relationship between the cutting forces and the overall friction coefficient $\mu_{a p}$. As in the Merchant's analysis [7], we consider the equilibrium of the forces exerted on the chip domain above the segment $A B_{A}$ (domain $D_{2}$ introduced in Section 8), see Fig. $1 \mathrm{~b}$. The cutting and feed forces are obtained as

$F_{C}=\frac{t_{1} \bar{\tau}_{P Z}}{\sin \phi_{A}} \frac{\cos \left(\lambda_{a p}-\alpha\right)}{\cos \left(\lambda_{a p}-\alpha+\phi_{A}\right)}$

$F_{T}=\frac{t_{1} \bar{\tau}_{P Z}}{\sin \phi_{A}} \frac{\sin \left(\lambda_{a p}-\alpha\right)}{\cos \left(\lambda_{a p}-\alpha+\phi_{A}\right)}$

The mean shear flow stress along $A B_{A}$ is taken as the mean shear flow stress $\bar{\tau}_{P Z}$ within the primary shear zone. The apparent friction angle $\lambda_{a p}$ accounts for the global response of the tool-chip interface. Relationships (25)-(26) are only first approximations of the cutting force components since solely the portion $A J$ of the toolchip contact is comprised in the domain $D_{2}$ (clearance contact excluded).

In Fig. $18 \mathrm{c}$ and $\mathrm{d}$ the cutting and feed forces obtained with Finite Element calculations are compared to the values resulting from the analytical relationships (25) and (26). In Eqs. (25) and (26) values of $\bar{\tau}_{P Z}, \lambda_{a p}\left(\mu_{a p}\right)$ and $\phi_{A}$ are obtained from Table 4 . A good correlation is found, showing the consistency of the calculations. Moreover, the effects of friction, shear flow stress and shear angle $\phi_{A}$ on forces can be evaluated separately, which is hardly feasible by Finite Element calculations.

Eqs. (25)-(26) show how $F_{C}$ and $F_{T}$ are affected by the apparent friction $\mu_{a p}$. This coefficient enters in a direct way in Eqs. (25)-(26), but also in an indirect manner through $\phi_{A}$. Indeed, the shear angle is strongly dependent upon the overall friction coefficient $\mu_{a p}$. The indirect contribution of $\mu_{a p}$ through $\phi_{A}$ is quantified in Fig. 19a by holding $\phi_{A}$ constant (upper curve with crosses and lower curve with squares) and comparing with the total variation of $F_{C}$ given by Eq. (25) where $\phi_{A}$ is left free to vary (bold line with small circles). The cutting conditions are those of Table 4 and the sliding friction coefficient is $\mu=0.8$. The reference curve (bold line with small circles) represents the variation of $F_{C}$ with $V$ as predicted by Eq. (25) where parameters vary according to the data of tests \#51-60 reported in Table 4. The upper curve (with crosses) corresponds to the constant value of the shear angle $\phi_{A}(V=$ $\left.1 \mathrm{~ms}^{-1}\right)=9.7^{\circ}$ associated to the cutting speed $V=1 \mathrm{~ms}^{-1}$. The variation of $F_{C}$ appears to be weak when $\phi_{A}$ is maintained at the constant value $9.7^{\circ}$. The lower curve (with squares) corresponds to 

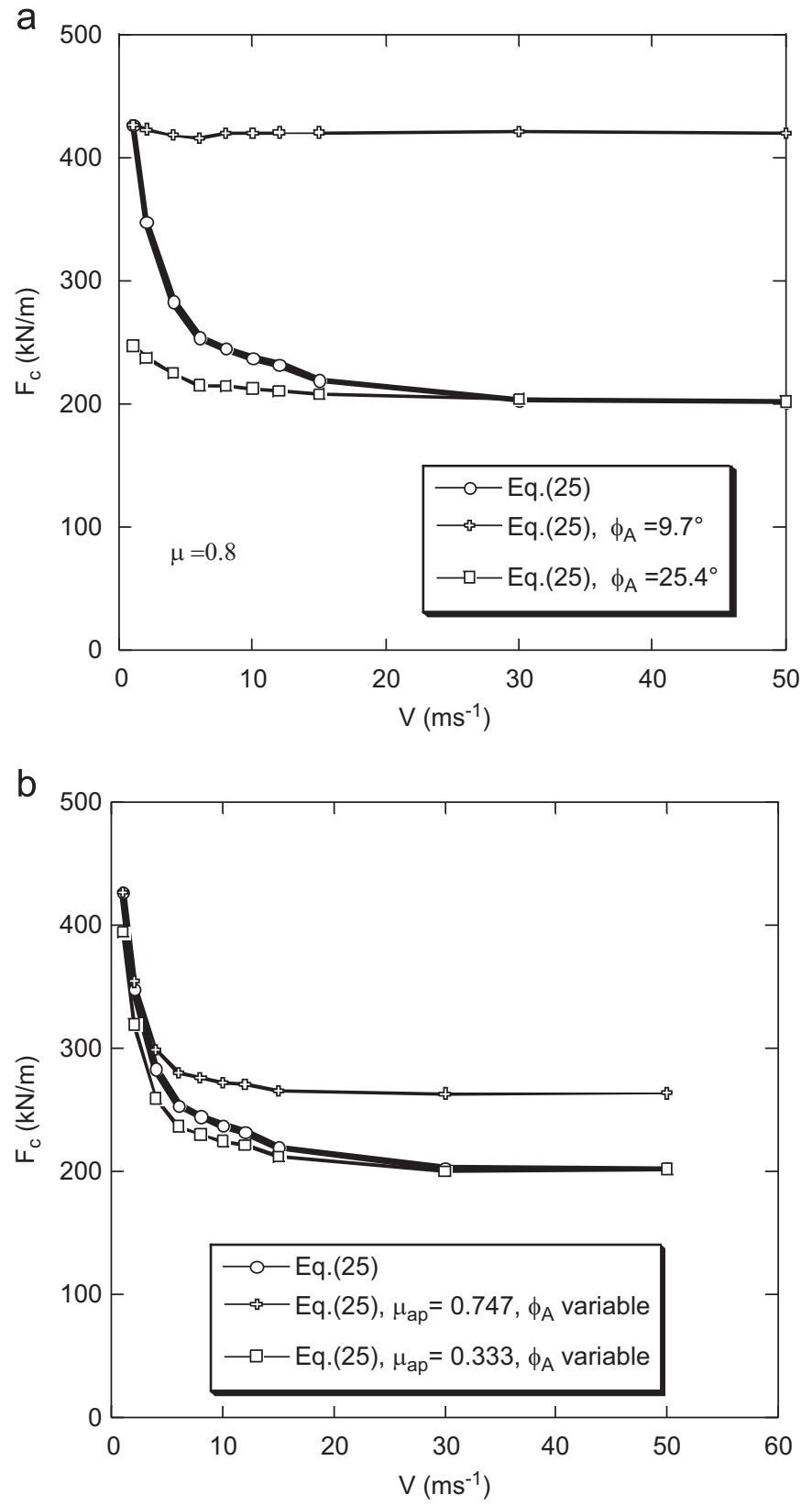

Fig. 19. Evolution of the cutting force $F_{C}$ with respect to the cutting speed $V . F_{C}$ is evaluated with Eq. (25) and the data displayed in Table 4 for $\mu=0.8$. (a) The effect of the shear angle is quantified by holding $\phi_{A}$ constant in Eq. (25). The reference curve (bold solid line with circles) is obtained by leaving $\phi_{A}$ free to vary with $V$. (b) The direct effect of $\mu_{a p}\left(\lambda_{a p}\right)$ is analyzed by holding $\mu_{a p}$ constant in Eq. (25) and comparing with the reference curve.

the fixed value of the shear angle $\phi_{A}\left(V=50 \mathrm{~ms}^{-1}\right)=25.4^{\circ}$ associated to the cutting speed $V=50 \mathrm{~ms}^{-1}$. Here again, a small variation of $F_{C}$ is observed which is mainly due to the direct effect of $\mu_{a p}$ in Eq. (25) (indirect effect of $\mu_{a p}$ through $\phi_{A}$ is excluded). As $\mu_{a p}$ weakens with $V$, the cutting force is decreasing as well, but insufficiently with respect to the large drop of the force observed for the reference curve.

The direct effect of $\mu_{a p}$ on $F_{C}$ is quantified in Fig. 19b. The upper curve (crosses) represents the variation of $F_{C}$ when $\mu_{a p}$ is maintained in Eq. (25) at the constant value $\mu_{a p}=0.747$ corresponding to $V=1 \mathrm{~ms}^{-1}$. $\phi_{A}$ varies according to the values reported in Table 4. Similarly, the lower curve is associated to $\mu_{a p}=0.333$ corresponding to $V=50 \mathrm{~ms}^{-1}$. From these results it is clear that the variation of $F_{C}$ with $V$ is mostly controlled by $\phi_{A}$.

From the examination of the results reported in Table 4 , it appears that $\bar{\tau}_{P Z}$ is weakly sensitive to cutting conditions. Therefore, the cutting force $F_{C}$ and the thrust force are just slightly affected by the variation of $\bar{\tau}_{P Z}$ due to cutting conditions.

\subsection{Forces exerted on the tool rake face}

The normal and tangential components of the force exerted by the chip on the tool rake face IJ (see Fig. 1b) can be calculated by considering the equilibrium of external forces applied on the boundaries of the chip domain $D_{1}$ introduced in Section 8 (domain above the line $\left.I B^{*}\right)$. The following result is obtained:

$F_{n}($ rake $)=\frac{\left(t_{1}-R\right) \bar{\tau}_{P Z}}{\sin \phi^{*}} \frac{\cos (\bar{\lambda})}{\cos \left(\bar{\lambda}+\phi^{*}\right)}$

$F_{t}($ rake $)=\frac{\left(t_{1}-R\right) \bar{\tau}_{P Z}}{\sin \phi^{*}} \frac{\sin (\bar{\lambda})}{\cos \left(\bar{\lambda}+\phi^{*}\right)}$

It is worth noticing that the uncut chip thickness $t_{1}$ which appeared in Eqs. (25)-(26) is replaced in relationships (27)-(28) by $t_{1}-R$. This is related to the fact that the bottom of $D_{2}$ is $A$ (vertical distance to the workpiece free-surface nearly equal to $t_{1}$ ) while the bottom of domain $D_{1}$ is $J$ (vertical distance to the workpiece free surface is equal to $\left.t_{1}-R\right)$.

The evolutions of $F_{n}($ rake $)$ and of $F_{t}$ (rake) in terms of the cutting speed are displayed in Fig. 20 for $\mu=0.2$ and 0.8 and the cutting conditions associated to Table 4. The results obtained from Eqs. (27)-(28), with values of $\bar{\tau}_{P Z}, \phi^{*}$ and $\bar{\lambda}$ given by Table 4 , are found to be in excellent agreement with Finite Element calculations of $F_{n}($ rake $)$ and of $F_{t}($ rake $)$. Using the shear angle $\phi=\arctan \left(t_{1} / t_{2}\right)$ in place of $\phi^{*}$ in Eqs. (27)-(28) would lead to similar results but with lower quality (represented by crosses in Fig. 20).

It should be mentioned that $\bar{\tau}_{P Z}$ in Eqs. (25) and (26) corresponds to the mean shear stress exerted on the segment $A B_{A}$ while $\bar{\tau}_{P Z}$ in Eqs. (27) and (28) is related to $I B^{*}$. However, the same value of $\bar{\tau}_{P Z}$ (given in Table 4) has been used in all calculations. This point is justified in Appendix E.

\subsection{Effect of the Taylor-Quinney coefficient}

The effect on $F_{C}$ of the Taylor-Quinney coefficient $\beta$ (conversion of plastic work into heat) is quantified in Fig. 21. The evolution of the cutting force $F_{C}$ with respect to the cutting speed is reported in Fig. 21a for various values of $\beta$. The variations of $F_{C}$ follow the same trends as those reported for $\bar{\mu}$ and $\mu_{a p}$, respectively in Figs. 6 and $9 \mathrm{~b}$. In particular, the cutting force $F_{C}$ is a decreasing function of $\beta$ for a fixed value of the cutting speed. However, contrarily to $\mu_{a p}$ or $\bar{\mu}$, the forces $F_{C}$ and $F_{T}$ remain significantly affected by $\beta$ at low cutting speeds. Results are shown in Table 8 for the low cutting speed $V=2 \mathrm{~ms}^{-1}$ and $\mu=0.6$. It is worth observing that both $\bar{\tau}_{S Z}$ (characterizing the material resistance to flow) and the apparent friction coefficient $\mu_{a p}$ appear to be weakly affected by $\beta$. Thus, according to Eqs. (25)-(26) the dependence of $F_{c}$ and $F_{T}$ upon $\beta$ should solely result from the variation of the shear angle $\phi_{A}\left(10^{\circ}\right.$ for $\beta=0.6 ; 13.3^{\circ}$ for $\beta=0.9$ ).

\section{Conclusions}

For the modeling of machining processes, it is essential to characterize local fields and global variables and to analyze their interactions. Two robust and independent approaches have been used in that purpose. The first is purely numerical and based on an Arbitrary Lagrangian Eulerian Finite Element model. The second is 

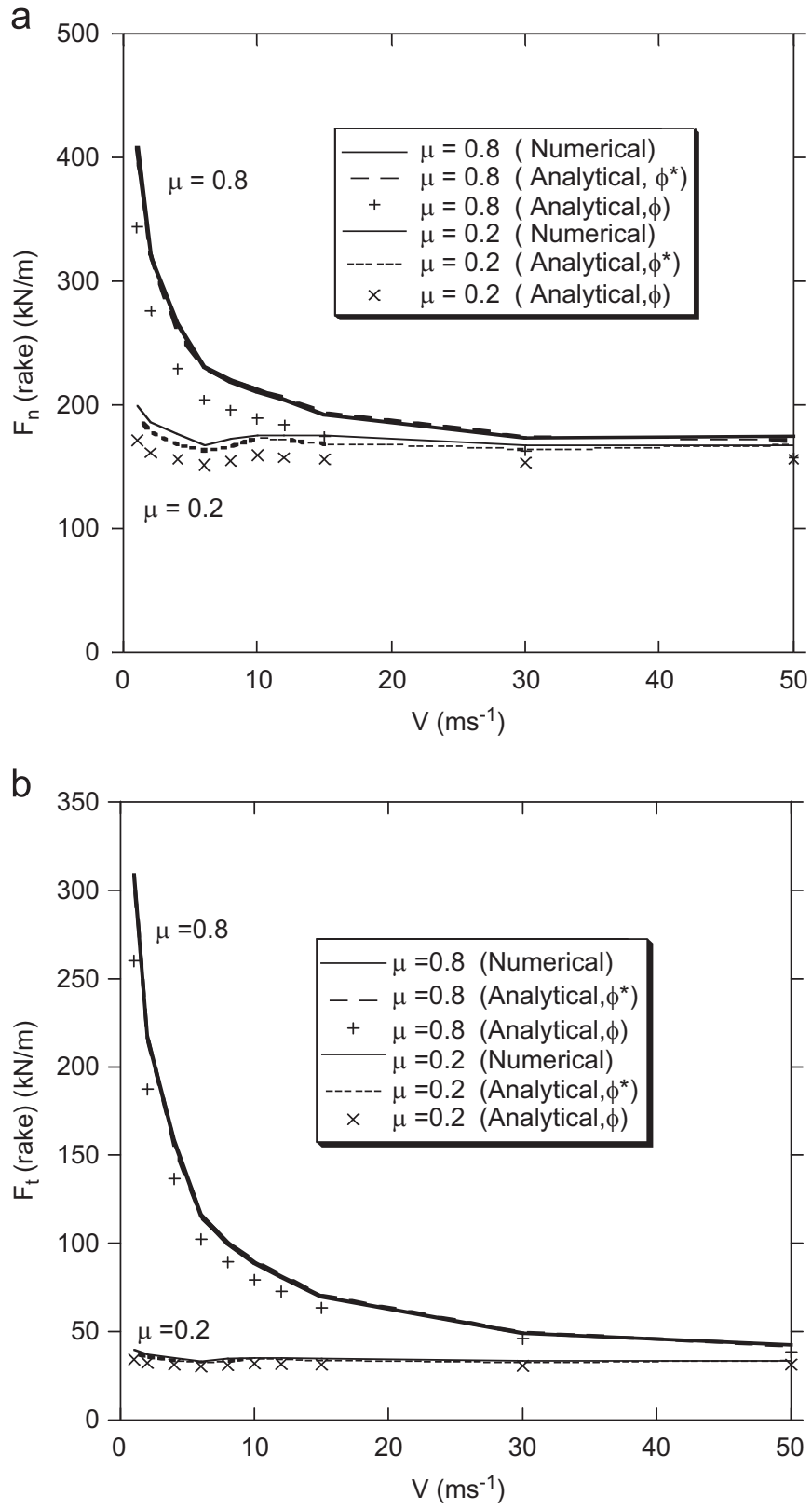

Fig. 20. (a) Normal force $F_{n}$ (rake) and (b) tangential force $F_{t}$ (rake) exerted on the tool rake-face $I J$ (see Fig. 1b) versus cutting speed for low and high values of sliding friction, resp. $\mu=0.2$ and 0.8 . Cutting conditions are those of Table 4 . Numerical values of $F_{n}($ rake $)$ and $F_{t}($ rake $)$ are taken from Table 4 and are compared to analytical results provided by resp. Eqs. (27) and (28).

grounded on analytical means. A parallel was systematically drawn between these approaches in such a way that numerical data became more meaningful as they could be put in relation to conceptual models of the phenomena studied.

The overall friction properties characterizing the tool-chip contact in orthogonal cutting, have been studied. The global response of the tool rake face was described by a mean friction coefficient $\bar{\mu}$ while the response of the tool-chip interface in its totality (including the tool edge and the clearance contact) was represented by the apparent friction coefficient $\mu_{a p}$.

A simple formulation of contact based on the Coulomb friction law and a constant sliding friction coefficient was used in this work. Nevertheless, numerical results have shown that the sliding and sticking contact regimes could be well described within this framework. Along the sticking zone, the shear stress was found to be equal
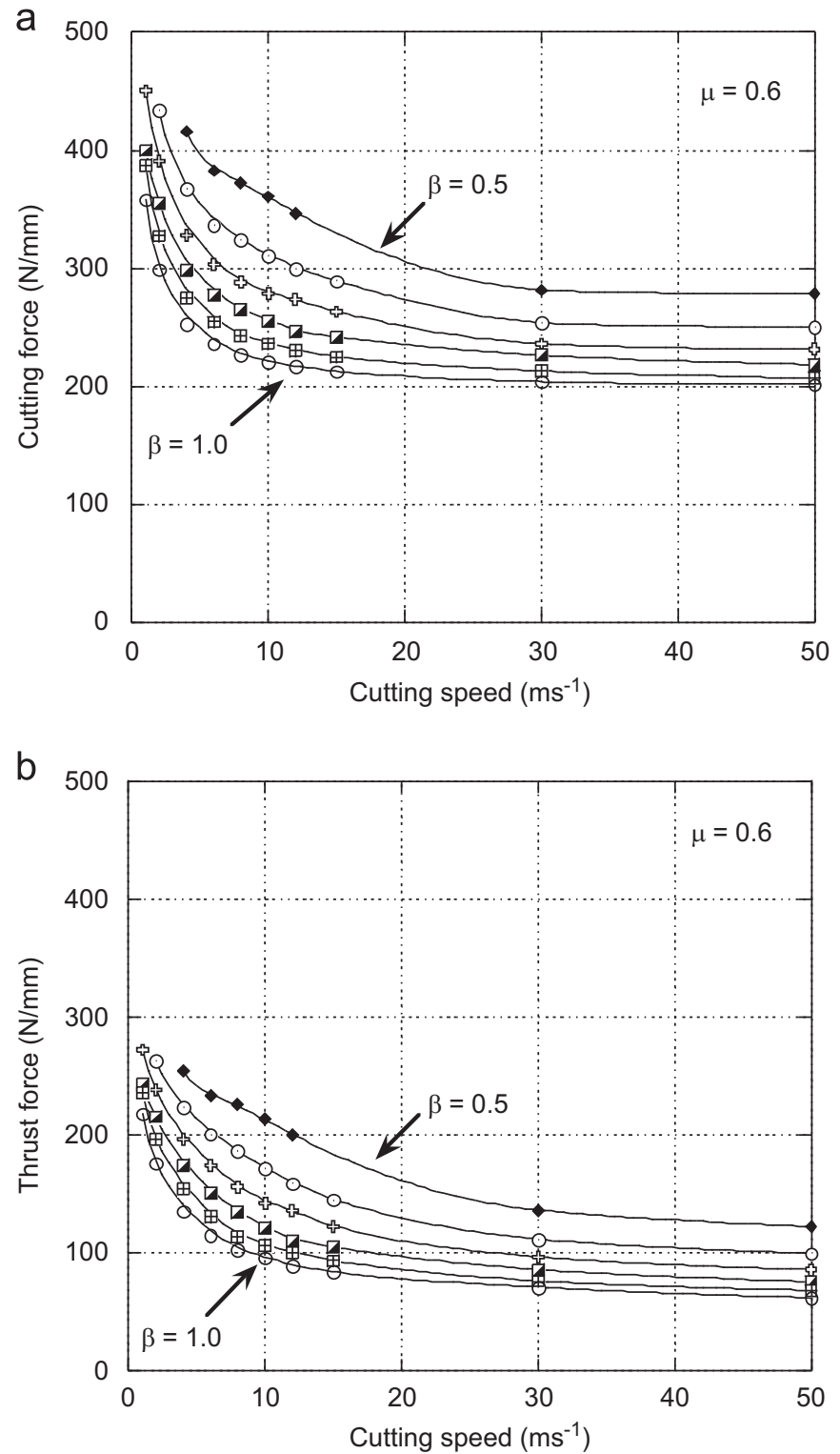

Fig. 21. Effect of the coefficient $\beta$ of conversion of the plastic work into heat on (a) the cutting force $F_{C}$ and (b) the thrust force $F_{T}$. Cutting conditions are those of Table 4 for $\mu=0.6$.

to the shear flow stress of the work material. Interesting correlations were observed with respect to experimental features. The softening of overall friction coefficients with the cutting speed was found to reproduce accurately the experimental trends for the steel (workmaterial) and the carbide tools considered here. The effect of the feed on the overall friction response was also well accounted for.

The overall friction characteristics appeared to be the result of the combined effects of sliding and sticking contact. Sticking occurs in the vicinity of the tool tip and is controlled by the magnitude of the shear flow stress of the work material which is itself governed by the chip temperature at the tool rake face. When the cutting speed was increased, a transition was found from a sliding regime towards a sticking dominated contact.

For high values of the sliding friction coefficient $\mu$, sticking dominates the overall response of the tool-chip interface for the whole range of cutting speeds considered here $\left(1 \mathrm{~ms}^{-1} \leq V \leq\right.$ $\left.50 \mathrm{~ms}^{-1}\right)$. Then, the decay of $\mu_{a p}$ and $\bar{\mu}$ is solely due to thermal softening of the work material in the sticking zone and the effect of $\mu$ is wiped out as soon as contact is dominated by sticking. 
Table 8

Effect of the Taylor-Quinney coefficient $\beta$ on cutting parameters and forces for the cutting conditions: $\mu=0.6, R=0.015 \mathrm{~mm}, t_{1}=0.1 \mathrm{~mm}, V=2 \mathrm{~ms}{ }^{-1}$.

\begin{tabular}{|c|c|c|c|c|c|c|c|}
\hline$\beta$ & $\mu_{a p}$ & $\phi_{A}$ (deg.) & $\bar{\tau}_{P Z}(\mathrm{MPa})$ & $\begin{array}{l}F_{C}(\mathrm{kN} / m) \\
\text { (Numerical) }\end{array}$ & $\begin{array}{l}F_{C}(\mathrm{kN} / m) \\
\text { Eq. }(25)\end{array}$ & $\begin{array}{l}F_{T}(\mathrm{kN} / m) \\
\text { (Numerical) }\end{array}$ & $\begin{array}{l}F_{T}(\mathrm{kN} / m) \\
\text { Eq. }(26)\end{array}$ \\
\hline 0.6 & 0.606 & 10 & 621 & 434 & 407 & 263 & 246 \\
\hline 0.9 & 0.605 & 13.3 & 624 & 339 & 325 & 205 & 197 \\
\hline
\end{tabular}

For small values of $\mu$, the quantities $\mu_{a p}$ and $\bar{\mu}$ are weakly dependent upon $V$ since sticking is negligible and thereby thermal softening of the bulk material has no effect on overall friction characteristics.

When contact is dominated by sticking, it appeared possible to formulate a law describing the dependence of the mean friction coefficient $\bar{\mu}$ with respect to the main factors controlling its evolution (shear flow stress of the work material, average normal stress, sticking length ratio). Essentially, $\bar{\mu}$ appeared as the ratio of the shear flow stress by the averaged normal stress. The difference between $\bar{\mu}$ and the apparent friction coefficient $\mu_{a p}$ results from the effect of the tool edge-radius $R$. The effects of $R$ and of the uncut chip thickness on $\mu_{a p}-\bar{\mu}$ have been quantified. Finally, it was shown that the evolutions of $\mu_{a p}$ and of $\bar{\mu}$ in terms of cutting conditions (cutting speed and uncut chip thickness, the rake angle was taken as zero) could be represented with phenomenological constitutive laws depending solely on a characteristic temperature of the chip at the tool rake face.

Having a better perception of the relationship between global friction characteristics and local variables has not only a theoretical interest but offers also important practical implications. Firstly, it should be noted that only global friction characteristics are directly accessible from orthogonal cutting experiments. Knowing the relationship between the local value $\mu$ of the sliding friction coefficient and global friction characteristics is helpful for deducing the value of $\mu$, see [39]. In that perspective, it was important to clarify the effects of the cutting speed, feed, tool edge radius and $\mu$ on global friction characteristics.

Another prospect is provided by the development of analytical models of metal cutting processes. This aspect offers interesting perspectives for the present research work. A central issue for the calculation of cutting and thrust forces, contact length and shear angle is related to the capability of describing the global frictional response of the tool-chip interface which is a salient aspect of this work.

Tool-chip contact length and contact forces exerted onto the tool were also characterized by numerical and analytical means and compared to experimental data. In particular, it was investigated how the contact length between tool and chip is correlated to global friction, shear angle, uncut chip thickness and to the profile of the normal stress on the tool-chip interface.

In the present work, the sliding friction coefficient was supposed to be constant i.e. to be independent from cutting conditions. Despite this simplifying assumption, it appeared that many experimental features could be well described for the work material considered here (medium carbon steel 42CrMo4). However, there are some experimental evidences that the sliding friction coefficient might be influenced in certain cases by contact conditions (sliding velocity, contact pressure, contact temperature) [40-41]. The functional dependence of the sliding friction coefficient with respect to contact conditions and the implications in the modeling of machining are the object of a current work.

\section{Acknowledgments}

AM acknowledges the support of Carlos III University with a Catedra de Excelencia funded by Banco Santander.

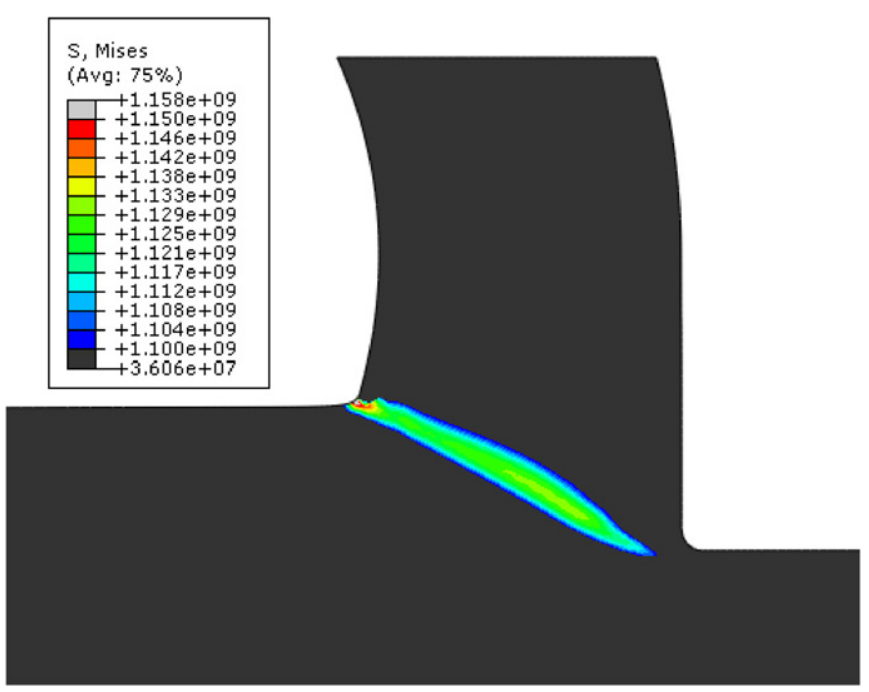

Fig. 22. Distribution of the Mises equivalent stress in the center of the primary shear zone.

\section{Appendix A. Characterization of the average shear flow stress} $\bar{\tau}_{P Z}$ along the primary shear zone

The method for characterizing $\bar{\tau}_{P Z}$ is illustrated by considering Fig. 22 showing the distribution of the Von-Mises equivalent stress $\sigma_{e q}$ in the deformed workpiece. The cutting conditions are those of test \#58 of Table $4\left(\mu=0.8, V=15 \mathrm{~ms}^{-1}\right)$. A lower cut-off limit, $\sigma_{e q}^{\lim }=1100 \mathrm{MPa}$, has been considered in Fig. 22. Thus, the variation of $\sigma_{e q}$ is only displayed in the range: $\sigma_{e q}^{\text {lim }} \leq \sigma_{e q} \leq \sigma_{e q}^{\max }$, with $\sigma_{e q}^{\max }=1158 \mathrm{MPa}$. This region represents the part of the primary shear zone where the chip is mostly formed by simple shearing. According to the $J_{2}$-flow theory, the shear flow stress is given for simple shearing by: $\bar{\tau}_{P Z}=\left(\bar{\sigma}_{e q} / \sqrt{3}\right)$. Here, $\bar{\sigma}_{e q}=\left(\sigma_{e q}^{\max }+\sigma_{e q}^{l i m} / 2\right)$ represents the average value of $\sigma_{e q}$ in the shear band domain shown in Fig. 22. It is worth noting that the amplitude of variation of $\sigma_{e q}$ within this band is only 5\%. For the test \#58 it is found that $\bar{\tau}_{P Z}=650 \mathrm{MPa} \cdot \bar{\tau}_{P Z}$ is taken as the mean flow stress in the primary shear zone.

\section{Appendix B. Characterization of the stress exponent $\xi$}

The stress distribution along the rake face $0 \leq x \leq l_{c}$ is represented in Fig. 23 for cutting conditions of Table 4 and $\mu=0.8$, $V=8 \mathrm{~ms}^{-1}$. The distribution given by Eq. (11) (dashed line) is compared to Finite Element calculations (solid line). The stress exponent in Eq. (11) is characterized as $\xi=0.23$ and the scaling factor has the value $\sigma_{0}=1150 \mathrm{MPa}$. The correspondence between numerical data and Eq. (11) is rather good along $66 \%$ of the contact length and appears to be less accurate in the last part of the contact. However, Eq. (11) is mainly used for calculating force moments with respect to the tool tip or stress average along the contact length. The representation given by Eq. (11) appears to be sufficient for that purpose. 


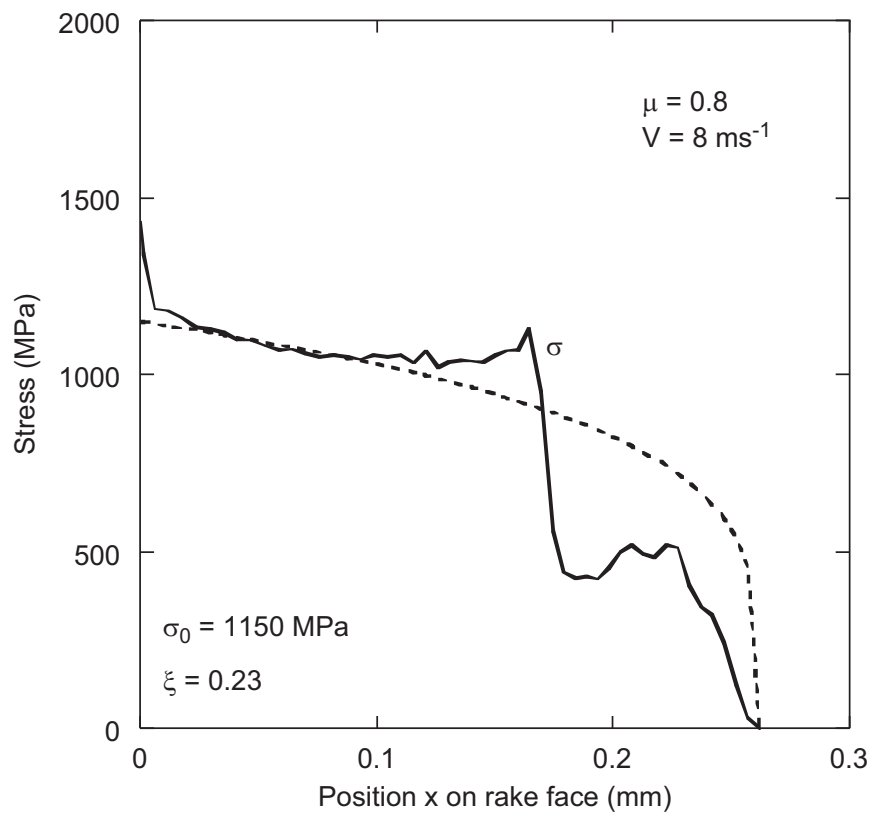

Fig.23. Distribution of the normal stress along the rake face and representation with the analytical expression Eq. (11) with stress exponent $\xi=0.23$.

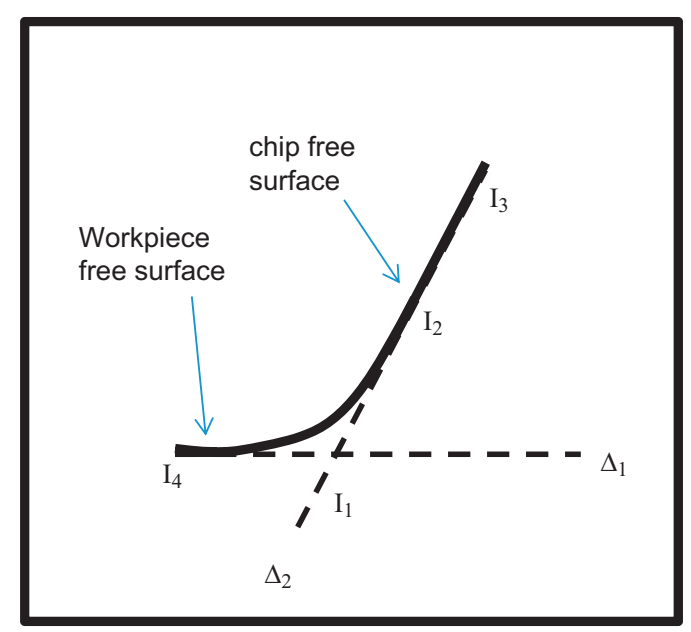

Fig. 24. Schematic view of the transition between the free-surface of the workpiece and the chip free-surface.

\section{Appendix C}

A zoom of the chip free-surface in the vicinity of the primary shear zone is displayed in Fig. 24. The point $I_{1}$ is at the intersection of two straight lines $\Delta_{1}$ and $\Delta_{2}$ defined as follows. $\Delta_{1}$ is the horizontal line aligned with the workpiece surface. In general, it is observed that the transition between the workpiece surface and the chip is made by a curve $I_{4} I_{2}$ followed by a straight segment $I_{2} I_{3}$ of small extension on the chip free-surface, see Fig. 24. Beyond $I_{3}$ the chip starts to curl. $\Delta_{2}$ is the straight line aligned with the segment $I_{2} I_{3}$.

\section{Appendix D}

It would be hard to calculate analytically the moment of the contact forces exerted on the domain $D_{2}$ by accounting for the real stress distribution around the tool edge. A heuristic and easiest way to proceed consists in developing the line $J A$ into a straight segment
$J A^{\prime}$ by projection onto the axis $I x$ aligned with the tool rake face, see Fig. $1 \mathrm{~b}$. The length of this projected line is nearly equal to $l_{c}+R$, where $R$ is the tool edge radius. It is assumed that the distribution of the normal stress along the line $J A^{\prime}$ has the form given by relationship Eq. (11)

$\sigma\left(x^{\prime}\right)=\sigma_{0}\left(1-\frac{x^{\prime}}{l_{c}+R}\right)^{\xi_{A}}$

However, the origin of the $x$-axis is now taken at $A^{\prime}$. The corresponding coordinate is denoted as $x^{\prime}$ and the distance from $A^{\prime}$ to the end $J$ of contact is $l_{c}+R$. The stress exponent has the effective value $\xi_{A}$ which is a priori different from $\xi$ characterizing the stress distribution along $I J$ (the stress profile is more peaked at the tool edge). The moment with respect to $A$ of the contact forces exerted on the tool-chip interface $A J$ is evaluated by considering the moment with respect to $A^{\prime}$ (orthogonal projection of $A$ onto the axis $I X$ ) of the normal stress distribution Eq. (D1). Equilibrium of the moments with respect to $A$ of all the contact forces exerted on the boundary of the domain $D_{2}$ is considered. The following result can be derived by assuming that the normal stress is uniformly distributed on the segment $A B_{A}$ :

$\frac{l_{c}+R}{t_{1}}=\frac{\xi_{A}+2}{2} \frac{\sin \left(\phi_{A}+\lambda_{a p}-\alpha\right)}{\sin \left(\phi_{A}\right) \cos \left(\lambda_{a p}\right)}$

$\phi_{A}$ is the inclination angle of the plane $A I_{1}$ and $\lambda_{a p}=\arctan \left(\mu_{a p}\right)$ is the apparent friction angle. The apparent friction coefficient $\mu_{a p}$ defined by the relationship (5) is involved in the result (D2) since the resultant of the contact forces exerted by the tool on the boundary $A J$ of the domain $D_{2}$ is in first approximation $F_{C} \underline{e}_{Y}-F_{T} \underline{e}_{X}$ (if the clearance contact is neglected).

\section{Appendix E}

Let us denote by $\bar{\tau}(\omega)$ the mean shear stress applied on any segment slightly disoriented by an angle $\omega$ with respect to the primary shear zone (PSZ). We have $\bar{\tau}(0)=\bar{\tau}_{P Z}$ where $\bar{\tau}_{P Z}$ is the mean shear stress applied along the PSZ. $\bar{\tau}(\omega)$ is maximum for $\omega=0$ since the mode of deformation along the PSZ is simple shear. Considering the Taylor expansion of $\bar{\tau}(\omega)$ with respect to $\omega$, it can be written that $\bar{\tau}(\omega)=\bar{\tau}_{P Z}+\left(\omega^{2} / 2\right) \bar{\tau}^{\prime \prime}(0)\left(\bar{\tau}^{\prime}(0)=0\right)$. Thus, to the second order with respect to $\omega, \bar{\tau}(\omega)$ can be approximated by $\bar{\tau}_{P Z}$.

\section{References}

[1] Shirakashi T, Usui E. Simulation analysis of orthogonal metal cutting process. Journal of the Japan Society for Precision Engineering 1976;42:340-5.

[2] Strenkowski JS, Carroll JT. A finite element model of orthogonal metal cutting. ASME Journal of Engineering for Industry 1985;107:346-54.

[3] Sekhon GS, Chenot JL. Some simulation experiments in orthogonal cutting. Numerical Methods in Industrial Forming Processes 1992:901-6.

[4] Marusich TD, Ortiz M. Modeling and simulation of high-speed machining. International Journal for Numerical Methods in Engineering 1995;38:3675-94.

[5] Rakotomalala R, Joyot P, Touratier M. Arbitrary Lagrangian-Eulerian thermomechanical finite element model of material cutting. Communications in Numerical Methods in Engineering 1993;9:975-87.

[6] Olovsson L, Nilsson L, Simonsson K. An ALE formulation for the solution of two-dimensional metal cutting problems. Computers and Structures 1999;72: 497-507.

[7] Merchant ME. Mechanics of the metal cutting process I: orthogonal cutting. Journal of Applied Physics 1945;16:267-75.

[8] PLB Oxley. Mechanics of machining. Chichester, UK: Ellis Horwood; 1989.

[9] Molinari A, Dudzinski D. Stationary shear bands in high speed machining. Comptes Rendus de l'Académie des Sciences Série II 1992;315:399-405.

[10] Dudzinski D, Molinari A. A modeling of cutting for viscoplastic materials. International Journal of Mechanical Sciences 1997;39:369-89.

[11] Karpat Y, Özel T. Predictive analytical and thermal modeling of orthogonal cutting process. Part I: predictions of tool forces, stresses and temperature distributions. ASME Journal of Manufacturing Science and Engineering 2006;128:435-44.

[12] Childs THC. Friction modelling in metal cutting. Wear 2006;260(3):310-8. 
[13] Childs THC. Numerical experiments on the influence of material and other variables on plane strain continuous chip formation in metal machining. International Journal of Mechanical Sciences 2006;48:307-22.

[14] Childs THC, Dirikolu MH, Maekawa K. Modelling of friction in the simulation of metal machining. In: Proceedings of the 24th Leeds-Lyon symposium on tribology, Elsevier Tribology Series, vol. 34. Amsterdam, 1998, p. 337-46.

[15] Zorev NN. Inter-relationship between shear processes occurring along tool face and shear plane in metal cutting, International Research in Production Engineering. New York: ASME; 1963 pp. 42-49.

[16] Filice L, Micari F, Rizzuti S, Umbrello D. A critical analysis on the friction modelling in orthogonal machining. International Journal of Machine Tools \& Manufacture 2007;47(3-4):709-14.

[17] Özel T. Influence of friction models on finite element simulations of machining. International Journal of Machine Tools \& Manufacture 2006;46(5):518-30.

[18] Arrazola PJ, Özel T. Investigation on the effects of friction modeling in finite element simulation of machining. International Journal of Mechanical Sciences 2010;52:31-42.

[19] Sutter G, Molinari A. Analysis of the cutting force components and friction in high speed machining. Journal of Manufacturing Science and Engineering 2005; $127: 245-50$.

[20] Molinari A, Moufki A, Dudzinski D. Study on behaviour of 42CrMo4 Steel. Final technical report, CREAS Ascometal, 1997.

[21] Hibbit, Karlsson \& Sorenson Inc. ABAQUS analysis user's manual, Version 6.4, 2003

[22] Devillez A, Lesko S, Mozer W. Cutting tool crater wear measurements with white light interferometry. Wear 2004;256:56-65.

[23] Albrecht P. New developments in the theory of the metal-cutting process. Journal of Engineering for Industry 1960:348-58.

[24] Gioia G, Ortiz M. The two-dimensional structure of dynamic boundary layers and shear bands in thermoviscoplastic solids. Journal of Mechanics and Physics of Solids 1996;44:251-92.

[25] Rittel D, Ravichandran G, Venkert A. The mechanical response of pure iron at high strain rates under dominant shear. Materials Sciences and Engineering A 2006;432:191-201.

[26] Ravichandran G, Rosakis AJ, Hodowany J, Rosakis P. On the conversion of plastic work into heat during high-strain-rate deformation. In: Furnish MD, Thadhani NN, Horie Y, editors. CP620 shock compression of condensed matter-2001. American Institute of Physics; 2002. p. 557-62.

[27] Sutter G, Molinari A, Faure L, Klepaczko JR, Dudzinski D. An experimental study of high speed orthogonal cutting. ASME Journal of Manufacturing Science and Engineering 1998;120:169-72.
[28] Moufki A, Molinari A, Dudzinski D. Modelling of orthogonal cutting with a temperature dependent friction law. Journal of Mechanics and Physics of Solids 1998;46:2103-38

[29] Sadik MI, Lindström B. The effect of restricted contact length on too performance. Journal of Materials Processing Technology 1995:48:275-82.

[30] Toropov A, Ko SL. Prediction of tool-chip contact length using a new slip-line solution for orthogonal cutting. International Journal of Machine Tools and Manufacture 2003;43:1209-15.

[31] Gad GS, Armarego EJA, Smith AJR. Tool-chip contact length in orthogonal machining and its importance in tool temperature predictions. International Journal of Production Research 1992;30:485-501.

[32] Sutter G, Ranc N. Temperature fields in a chip during high-speed orthogonal cutting-an experimental investigation. International Journal of Machine Tools and Manufacture 2007;47:1507-17.

[33] Lee EH, Shaffer BW. The theory of plasticity applied to a problem of machining. Journal of Applied Mechanics 1951;18:405-13.

[34] Abuladze NG. Character and the length of tool-chip contact. In: Proceedings of the machinability of heat-resistant and titanium alloys, Kuibyshev 1962 p. 68-78 [in Russian].

[35] Poletika MF. Contact loads on tool faces. Machinostronie, Moscow, 1969 [in Russian].

[36] Marinov VR. Hybrid analytical-numerical solution for the shear angle in orthogonal metal cutting. Part II. Experimental verification. International Journal of Mechanical Sciences 2001;43:415-26.

[37] Toropov A, Ko SL. Prediction of tool-chip contact length using a new slip-line solution for orthogonal cutting. International Journal of Machine Tools and Manufacture 2003;43:1209-15.

[38] Kato S, Yamaguchi K, Yamada M. Stress distribution at the interface between tool and chip in machining. Journal of Engineering for Industry 1972;94:683-9.

[39] Ozlu E, Budak E, Molinari A. Analytical and experimental investigation of rake contact and friction behavior in metal cutting. International Journal of Machine Tools and Manufacture 2009;49:865-75.

[40] Sutter G, Philippon S, Molinari A. An experimental investigation of dry friction for a large range of sliding velocities. Matériaux et Techniques 2004, $\mathrm{N}^{\circ}$ Hors Série. p. 1-5.

[41] Bonnet C, Valiorgue F, Rech J, Claudin C, Hamdi H, Bergheau JM, Gilles P. Identification of a friction model-application to the context of dry cutting of an AISI 316L austenitic stainless steel with a TiN coated carbide tool International Journal of Machine Tools and Manufacture 2008;48:1211-223. 